\begin{tabular}{|c|c|}
\hline Title & Interaction of non-radially symmetric camphor particles \\
\hline Author(s) & Ei, Shin-Ichiro; Kitahata, Hiroyuki; Koyano, Y uki; Nagayama, Masaharu \\
\hline Citation & $\begin{array}{l}\text { Physica. D, Nonlinear phenomena, 366, 10-26 } \\
\text { https://doi.org/10.1016j.physd.2017.11.004 }\end{array}$ \\
\hline Issue Date & 2018-03-01 \\
\hline Doc URL & http:/hdl .handle.net/2115/76223 \\
\hline Rights & $\begin{array}{l}\text { (2017 This manuscript version is made avail able under the CC-BY-NC-ND } 4.0 \text { license } \\
\mathrm{http}: / / \text { reativecommons.org/icenses/by-nc-nd/4.0/ }\end{array}$ \\
\hline Rights(URL) & http://creativecommons.org/icenses/by-nc-nd/4.0/ \\
\hline Type & article (author version) \\
\hline File Information & Physica D366 10-26.pdf \\
\hline
\end{tabular}

Instructions for use 


\title{
Interaction of non-radially symmetric camphor particles
}

\author{
Shin-Ichiro Eia ${ }^{\mathrm{a}, *}$, Hiroyuki Kitahata ${ }^{\mathrm{b}}$, Yuki Koyano ${ }^{\mathrm{b}}$, Masaharu Nagayama ${ }^{\mathrm{c}}$ \\ ${ }^{a}$ Department of Mathematics, Faculty of Science, Hokkaido University, Sapporo 060-0810, Japan \\ ${ }^{b}$ Department of Physics, Chiba University, Chiba 263-8522, Japan \\ ${ }^{c}$ Research Institute for Electronic Science, Hokkaido University, Sapporo 001-0020, Japan
}

\begin{abstract}
In this study, the interaction between two non-radially symmetric camphor particles is theoretically investigated and the equation describing the motion is derived as an ordinary differential system for the locations and the rotations. In particular, slightly modified non-radially symmetric cases from radial symmetry are extensively investigated and explicit motions are obtained. For example, it is theoretically shown that elliptically deformed camphor particles interact so as to be parallel with major axes. Such predicted motions are also checked by real experiments and numerical simulations.
\end{abstract}

Keywords: camphor particle, interaction, non-radial symmetry, self-propelled system

\section{Introduction}

Spontaneous motions are one of the most attractive behaviors both from phenomenological and theoretical points of view (e.g. [1,2,3] and the references in them). Traveling pulses are typical examples and extensive research has been conducted in this area (e.g. $[4,1,5,6,7])$.

Recently, a camphor particle floating on water was considered and the occurrence of spontaneous motions of a camphor particle was reported theoretically and experimentally $[8,9,10,11$, $12,13,14,15,16,17,18]$. Among them, radially symmetric camphor particles have been mainly treated and it was shown that the spontaneous motion can occur due to the symmetry breaking of the profile of dissolved camphor molecules at the water surface even if the camphor particle is radially symmetric. In practice, such symmetry breaking of camphor concentration profile causes an asymmetrical surface tension around the particle and, therefore, spontaneous motions of a camphor particle appear as the bifurcating branch from a standing solution by a pitchfork bifurcation. This can be regarded as a typical example of traveling spot in $\mathbb{R}^{2}$.

On the other hand, an asymmetric camphor particle has been considered as a natural extension from symmetric particles. It was reported that for an elliptic camphor particle the spontaneous traveling motion in the minor-axis direction primarily appears both theoretically and numerically (e.g. $[14,15])$. They also investigated various parameter sets numerically and checked what kind of behaviors, such as a traveling motion, self-rotating motion, and so on, appear in each parameter

\footnotetext{
${ }^{*}$ Corresponding author. E-mail: Eichiro@math.sci.hokudai.ac.jp Preprint submitted to Physica D
} 
region. In particular, they suggested the existence of an appropriate parameter region in which an elliptic camphor particle can stand still.

In this study, we focus on the aforementioned fact and investigate the interaction between two standing asymmetric camphor particles as the first step to analyze multi-camphor particles. In fact, there has been a lot of research related to multi-camphor particles (e.g. [19, 20, 21, 22, $23,24,25,26,27,28])$ such as analysis of the jam of camphor particles on a circle (e.g. [28]) although almost all of them are related to the interaction in one-dimensional space.

On the other hand, only few analyses of the interaction between multi-camphor particles in two-dimensional space have been presented, and even so, only for the case of radially symmetric particles. In fact, in [13], the interaction between two radially symmetric camphor particles was shown to be repulsive in a mathematically rigorous way, in which the equation describing the motion of two interacting traveling camphor particles with sufficiently small velocities was derived.

Here, we consider the interaction between non-radially symmetric camphor particles, but use the interaction of standing spot solutions as the first step to deal with multi-camphor particles with non-radially symmetric shapes.

We only consider two camphor particles. The considered model equation is

$$
\left\{\begin{aligned}
\tau_{1} \dot{\boldsymbol{p}}_{j} & =\int_{\partial \Omega_{j}(t)} \gamma(u) \boldsymbol{n} d s, \\
\tau_{2} \dot{\Theta}_{j} & =\int_{\partial \Omega_{j}(t)} \gamma(u)(\boldsymbol{r} \times \boldsymbol{n}) d s, \\
\partial_{t} u & =d \Delta u-\alpha u+a_{0}\left(\chi_{\Omega_{1}(t)}(\boldsymbol{x})+\chi_{\Omega_{2}(t)}(\boldsymbol{x})\right)
\end{aligned}\right.
$$

for $j=1,2$, where all coefficients $\tau_{j}, d, \alpha$, and $a_{0}$ are all positive constants. $\gamma(u)$ is a decreasing function, and $\chi_{A}(\boldsymbol{x})$ is the characteristic function for a set $A \subset \mathbb{R}^{2}$. Here, $u(t, \boldsymbol{x})$ denotes the surface concentration of the camphor molecular layer on water surface. Camphor particles are represented by $\Omega_{j}(t)$ with $\partial \Omega_{j}(t)=\left\{\boldsymbol{x} \in \mathbb{R}^{2} ; \boldsymbol{x}=\boldsymbol{p}_{j}(t)+\boldsymbol{r}\right\}$ as in Fig. 1, where $\boldsymbol{n}$ is the outward normal unit vector of $\partial \Omega_{j}(t), \boldsymbol{p}_{j}$ denote the locations of camphor particles, and $\Theta_{j}$ are the characteristic angles of the camphor particles. The vector product in two dimensions is defined as $\boldsymbol{r} \times \boldsymbol{n}=r_{1} n_{2}-r_{2} n_{1}$, where $\boldsymbol{r}={ }^{t}\left(r_{1}, r_{2}\right)$ and $\boldsymbol{n}={ }^{t}\left(n_{1}, n_{2}\right)$.

The physical meanings of Eq. (1.1) are as follows. The first and second equations represent balanced equations for the positions and the characteristic angles of camphor particles, respectively. The left-hand sides of both equations denote the resistance for translation and rotation, while the right-hand sides are the force and torque originating from the inhomogeneity of surface tension $\gamma(u)$. The constants $\tau_{1}$ and $\tau_{2}$ are the resistance coefficients for the translation and the rotation, respectively. The third equation represents the time evolution of concentration of camphor molecular layer, and the right-hand side is composed of the terms describing diffusion, sublimation into the air, and supply from the camphor particle. Here, $d, \alpha$, and $a_{0}$ are the diffusion constant, the sublimation rate, and supply rate from the camphor particle per unit area, respectively.

In real systems, the Marangoni convection is induced by the surface tension difference at water surface $[29,30,31,32]$. Therefore, we have to consider hydrodynamics to discuss the actual phenomenon in more detail. In the other place, one of the authors discuss that the effect of the hydrodynamic effect is regarded as effective diffusion when the camphor particle is moving with a sufficiently small velocity [33]. In this sense, our model does not precisely describe the motion of the camphor particle. However, since our model is constructed with simple equations, 


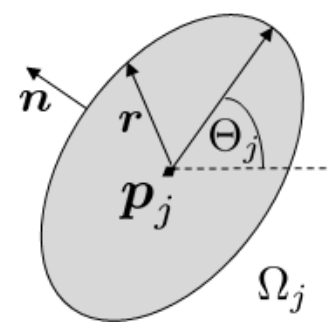

Figure 1: Camphor particle $\Omega_{j}$ with the location $\boldsymbol{p}_{j}$ and the characteristic angle $\Theta_{j}$.

and thus we consider our model can be adopted to the other phenomena such as chemotactic motion in which an object release chemicals from which the object tends to escape $[1,34,35]$.

For a single camphor particle with elliptic shape, the model equation was originally proposed in [15] as follows:

$$
\left\{\begin{aligned}
m \ddot{\boldsymbol{p}}+\tau_{1} \dot{\boldsymbol{p}} & =\int_{\partial \Omega(t)} \gamma(u) \boldsymbol{n} d s, \\
I \ddot{\Theta}+\tau_{2} \dot{\Theta} & =\int_{\partial \Omega(t)} \gamma(u)(\boldsymbol{r} \times \boldsymbol{n}) d s, \\
\partial_{t} u & =d \Delta u-\alpha u+a_{0} \chi_{\Omega(t)}(\boldsymbol{x}),
\end{aligned}\right.
$$

where $m \ddot{\boldsymbol{p}}$ and $I \ddot{\Theta}$ are inertia terms for translation and rotation, respectively. As mentioned above, it was shown in $[14,15]$ that an elliptic camphor particle tends to move in the minor-axis direction. It was also suggested by numerical simulations that a single elliptic camphor particle stands still when $\tau_{1}$ and $\tau_{2}$ are large. In this paper, we consider this case but with two identical elliptic camphors. In that case, each camphor particle does not move if it exists alone and hence, we can expect that the movements of two interacting camphor particles are rather slow. Thus, we neglect acceleration terms $\ddot{\boldsymbol{p}}$ and $\ddot{\Theta}$ in (1.2) and consider (1.1). It is noted that force-free and torque free conditions hold in Eq. (1.1), that is the surface tension working on the camphor particle balances the resistance force and torque.

By analyzing the interaction of them in (1.1), we can show that each camphor particle with elliptic shape moves and rotates so that their major axes are orthogonal to the center line connecting $\boldsymbol{p}_{1}$ and $\boldsymbol{p}_{2}$ if each camphor particle is slightly deformed from radial symmetry.

This paper is organized as follows: In Section 2, we present the model equation for a single particle that we treat in this paper precisely. It is not (1.1) but a slightly modified version. In Section 3 , the analysis of the interaction between two camphor particles is described. The validation of theoretical results using real experiments is described in Section 4. The theoretical results are also checked with numerical calculation and subsequently presented in Section 5.

\section{Preliminaries for (1.1) and a single camphor particle}

Before we deal with two camphor particles, we first consider a model of a single camphor 


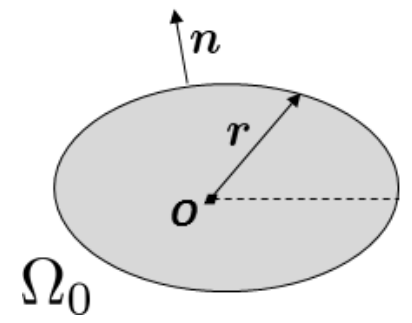

Figure 2: Fixed camphor particle $\Omega_{0}$ with the location at the origin $O$ and the characteristic angle $\Theta=0$.

particle. The model equation for a single camphor particle corresponding to (1.1) is

$$
\left\{\begin{aligned}
\tau_{1} \dot{\boldsymbol{p}} & =\int_{\partial \Omega(t)} \gamma(u) \boldsymbol{n} d s, \\
\tau_{2} \dot{\Theta} & =\int_{\partial \Omega(t)} \gamma(u)(\boldsymbol{r} \times \boldsymbol{n}) d s, \\
\partial_{t} u & =d \Delta u-\alpha u+a_{0} \chi_{\Omega(t)}(\boldsymbol{x}) .
\end{aligned}\right.
$$

Dividing the first and the second equations of (2.1) by $\tau_{1}$ and $\tau_{2}$, respectively, we consider

$$
\left\{\begin{aligned}
\dot{\boldsymbol{p}} & =\int_{\partial \Omega(t)} \gamma_{1}(u) \boldsymbol{n} d s, \\
\dot{\Theta} & =\int_{\partial \Omega(t)} \gamma_{2}(u)(\boldsymbol{r} \times \boldsymbol{n}) d s, \\
\partial_{t} u & =d \Delta u-\alpha u+a_{0} \chi_{\Omega(t)}(\boldsymbol{x}) .
\end{aligned}\right.
$$

Hereafter we assume $\gamma_{1}$ and $\gamma_{2}$ are certain decreasing functions but make no other assumptions, that is, $\gamma_{j}(u)$ are not necessarily equal to $\gamma(u) / \tau_{j}$ of $(1.1)$.

Let $R(\Theta):=\left(\begin{array}{cc}\cos \Theta & -\sin \Theta \\ \sin \Theta & \cos \Theta\end{array}\right)$ and $\Omega_{0}$ be a fixed camphor particle located at the origin $O$ and the characteristic angle $\Theta=0$ as in Fig. 2. Then $\Omega$ with the location $\boldsymbol{p}$ and the characteristic angle $\Theta$ is expressed by

$$
\Omega=\left\{\boldsymbol{x}=\boldsymbol{p}+R(\Theta) z ; \boldsymbol{z} \in \Omega_{0}\right\} .
$$

Then (2.2) is

$$
\left\{\begin{aligned}
\dot{\boldsymbol{p}} & =\int_{\partial \Omega_{0}} \gamma_{1}(u(t, \boldsymbol{p}+R(\Theta) \boldsymbol{z})) \boldsymbol{n} d s, \\
\dot{\Theta} & =\int_{\partial \Omega_{0}}^{\gamma_{2}}(u(t, \boldsymbol{p}+R(\Theta) z))(\boldsymbol{r} \times \boldsymbol{n}) d s, \\
\partial_{t} u & =d \Delta u-\alpha u+a_{0} \chi_{\Omega_{0}}(z),
\end{aligned}\right.
$$

for the solution $u=u(t, \boldsymbol{x})$ and the relation $\boldsymbol{x}=\boldsymbol{p}(t)+R(\Theta(t)) \boldsymbol{z}$.

Moreover, we introduce approximating smooth functions to the boundary integrals and the characteristic function as follows.

Let $\boldsymbol{K}_{0}(z) \in \mathbb{R}^{2}$ and $k_{0}(z) \in \mathbb{R}$ be smooth integral kernels satisfying

$$
\int_{\mathbb{R}^{2}} g(z) \boldsymbol{K}_{0}(z) d z=\int_{\partial \Omega_{0}} g(z) \boldsymbol{n} d s+O(\delta),
$$




$$
\int_{\mathbb{R}^{2}} g(z) k_{0}(z) d z=\int_{\partial \Omega_{0}} g(z)(\boldsymbol{r} \times \boldsymbol{n}) d s+O(\delta)
$$

for an arbitrary function $g(z)$ and a sufficiently small $\delta>0$. As a consequence, the model equation that we deal with is

$$
\left\{\begin{aligned}
\dot{\boldsymbol{p}} & =\int_{\mathbb{R}^{2}} \gamma_{1}(u(t, \boldsymbol{p}+R(\Theta) z)) \boldsymbol{K}_{0}(\boldsymbol{z}) d \boldsymbol{z} \\
\dot{\Theta} & =\int_{\mathbb{R}^{2}} \gamma_{2}(u(t, \boldsymbol{p}+R(\Theta) z)) k_{0}(\boldsymbol{z}) d \boldsymbol{z} \\
\partial_{t} u & =d \Delta u+f_{0}(u)+f_{1}(\boldsymbol{z})
\end{aligned}\right.
$$

where $z=R(-\Theta)(\boldsymbol{x}-\boldsymbol{p})$. Here $f_{1}(\boldsymbol{z})$ is also a smooth approximate function to $a_{0} \chi_{\Omega_{0}}(\boldsymbol{z})$ in $O(\delta)$ accuracy. Though $f_{0}(u)$ corresponds to $-\alpha u$, we do not necessarily make this assumption, but assume a general function satisfying $f(0)=0$ and $f_{0}^{\prime}(u)<0$ for $u \geq 0$.

In the following, we consider a stationary solution of (2.4). We first assume the existence of a stationary solution, say $S^{*}(\boldsymbol{x})$, satisfying

$$
\left\{\begin{aligned}
0 & =\int_{\mathbb{R}^{2}} \gamma_{1}\left(S^{*}(\boldsymbol{z})\right) \boldsymbol{K}_{0}(\boldsymbol{z}) d \boldsymbol{z}, \\
0 & =\int_{\mathbb{R}^{2}} \gamma_{2}\left(S^{*}(\boldsymbol{z})\right) k_{0}(\boldsymbol{z}) d \boldsymbol{z}, \\
0 & =d \Delta S^{*}+f_{0}\left(S^{*}\right)+f_{1}(\boldsymbol{x})
\end{aligned}\right.
$$

Then for any $\boldsymbol{p} \in \mathbb{R}^{2}$ and $\Theta \in \mathbb{R}, S(\boldsymbol{x} ; \boldsymbol{p}, \Theta):=S^{*}(R(-\Theta)(\boldsymbol{x}-\boldsymbol{p}))$ is a stationary solution of (2.4). Let $L:=d \Delta+f_{0}^{\prime}\left(S^{*}(\boldsymbol{x})\right)$ and assume the following:

(H1) $\left|S^{*}(\boldsymbol{x})\right| \leq c_{0} e^{-\alpha_{0}|\boldsymbol{x}|}$ for positive constants $c_{0}$ and $\alpha_{0}$;

(H2) the spectral set $\sigma(L) \subset\left\{\lambda \in \mathbb{C} ; \operatorname{Re}(\lambda)<-c_{1}\right\}$ for $c_{1}>0$.

Let $\bar{X}:=\mathbb{R}^{2} \times \mathbb{R} \times L^{2}\left(\mathbb{R}^{2}\right)$ with the inner product $\langle\bar{U}, \bar{V}\rangle_{\bar{X}}:=\langle\boldsymbol{p}, \boldsymbol{q}\rangle+\Theta \cdot \Xi+\langle u, v\rangle_{L^{2}}$ for $\bar{U}=$ ${ }^{t}(\boldsymbol{p}, \Theta, u)$ and $\bar{V}={ }^{t}(\boldsymbol{q}, \Xi, v) \in \bar{X}$. Let $\bar{S}(\boldsymbol{x} ; \boldsymbol{p}, \Theta):={ }^{t}(\boldsymbol{p}, \Theta, S(\boldsymbol{x} ; \boldsymbol{p}, \Theta)) \in \bar{X}$ and $\overline{\mathcal{L}}(\bar{U}) \in \bar{X}$ be the right-hand side of (2.4). Then $\overline{\mathcal{L}}(\bar{S}(\boldsymbol{x} ; \boldsymbol{p}, \Theta))=0$ holds. To investigate the stability of $\bar{S}(\boldsymbol{x} ; \boldsymbol{p}, \Theta)$, we consider the linearized operator of (2.4) with respect to $\bar{S}$. Let $\bar{L}(\boldsymbol{p}, \Theta):=\overline{\mathcal{L}}^{\prime}(\bar{S}(\cdot ; \boldsymbol{p}, \Theta))$ and $\bar{L}_{0}:=\overline{\mathcal{L}}^{\prime}(\bar{S}(\cdot ; \mathbf{0}, 0))$

Here we put $\boldsymbol{e}_{1}:={ }^{t}(1,0)$ and $\boldsymbol{e}_{2}:={ }^{t}(0,1)$ and express the angle derivative with respect to $\Theta$ by $\partial_{R} v:=\langle\nabla v, R(\pi / 2) z\rangle=\left(-z_{2} \partial_{z_{1}}+z_{1} \partial_{z_{2}}\right) v$ for $z=\left(z_{1}, z_{2}\right)$ and $v=v(z)$.

Proposition 2.1. The linearized operator $\bar{L}_{0}$ with respect to $\bar{S}(\boldsymbol{x} ; \mathbf{0} ; 0)$ is

$$
\overline{L_{0}} \bar{V}=\left(\begin{array}{c}
\int_{\mathbb{R}^{2}} \gamma_{1}^{\prime}\left(S^{*}(\boldsymbol{z})\right)\left(v+\left\langle\nabla S^{*}, \boldsymbol{q}\right\rangle+\Xi \partial_{R} S^{*}(\boldsymbol{z})\right) \boldsymbol{K}_{0}(\boldsymbol{z}) d \boldsymbol{z} \\
\int_{\mathbb{R}^{2}}^{\gamma_{2}^{\prime}\left(S^{*}(\boldsymbol{z})\right)\left(v+\left\langle\nabla S^{*}, \boldsymbol{q}\right\rangle+\Xi \partial_{R} S^{*}(\boldsymbol{z})\right) k_{0}(\boldsymbol{z}) d z} \\
L v-\left\langle\nabla f_{1}, \boldsymbol{q}\right\rangle-\Xi \partial_{R} f_{1}
\end{array}\right)
$$

for $\bar{V}={ }^{t}(\boldsymbol{q}, \Xi, v) \in \bar{X}$ and

$$
\bar{\Phi}_{1}:=\left(\begin{array}{c}
\boldsymbol{e}_{1} \\
0 \\
-\partial_{z_{1}} S^{*}
\end{array}\right), \bar{\Phi}_{2}:=\left(\begin{array}{c}
\boldsymbol{e}_{2} \\
0 \\
-\partial_{z_{2}} S^{*}
\end{array}\right), \bar{\Phi}_{3}:=\left(\begin{array}{c}
\mathbf{0} \\
1 \\
-\partial_{R} S^{*}
\end{array}\right)
$$


are the eigenfunctions of $\bar{L}_{0}$ associated with 0 eigenvalue.

This proposition is easily checked by direct calculations.

Then we have

Lemma 2.1. Suppose that assumptions (H1) and (H2) hold. If $\gamma_{1}$ and $\gamma_{2}$ are sufficiently small, then the spectral set $\sigma\left(\bar{L}_{0}\right)$ is given by $\sigma\left(\bar{L}_{0}\right)=\{0\} \cup \sigma_{1}$ with $\sigma_{1} \subset\left\{\lambda \in \mathbb{C} ; \operatorname{Re}(\lambda)<-c_{2}\right\}$ for $c_{2}>0$. Moreover, 0 is a semi-simple eigenvalue and $\operatorname{Ker} \bar{L}_{0}$ is just spanned by $\bar{\Phi}_{j}(j=1,2,3)$.

Proof.

Since $\gamma_{1}$ and $\gamma_{2}$ are sufficiently small, we introduce a small parameter $\epsilon>0$ and write $\gamma_{1}=\epsilon \bar{\gamma}_{1}$ and $\gamma_{2}=\epsilon \bar{\gamma}_{2}$. First, we show $\rho_{\epsilon} \subset \rho\left(\bar{L}_{0}\right)$, the resolvent set of $\bar{L}_{0}$, where $\rho_{\epsilon}:=$ $\left\{\lambda \in \mathbb{C} ; \operatorname{Re}(\lambda)>-c_{1},|\lambda| \geq c_{2}^{\prime} \epsilon\right\}$ for $c_{2}^{\prime}>0$. For $\lambda \in \rho_{\epsilon}$, we consider the equation

$$
\left(\lambda-\bar{L}_{0}\right) \bar{V}=\bar{G}
$$

for $\bar{V}={ }^{t}(\boldsymbol{q}, \Xi, v), \bar{G}={ }^{t}(\boldsymbol{a}, T, g)$, and $\boldsymbol{q}={ }^{t}\left(q_{1}, q_{2}\right)=q_{1} \boldsymbol{e}_{1}+q_{2} \boldsymbol{e}_{2}$. Defining $\bar{V}_{0}:=q_{1} \bar{\Phi}_{1}+q_{2} \bar{\Phi}_{2}+$ $\Xi \bar{\Phi}_{3}$, we see $\bar{V}=\bar{V}_{0}+\bar{V}_{1}$ with $\bar{V}_{1}={ }^{t}\left(\mathbf{0}, 0, v_{1}\right)$. Since $\bar{L}_{0} \bar{V}_{0}=0$ holds, by the substitution $\bar{V}=\bar{V}_{0}+\bar{V}_{1}$ into (2.6), we have

$$
\lambda \bar{V}_{0}+\left(\lambda-\bar{L}_{0}\right) \bar{V}_{1}=\bar{G}
$$

(2.7) supplies the equations

$$
\begin{aligned}
\lambda \boldsymbol{q}-\epsilon \int_{\mathbb{R}^{2}} \bar{\gamma}_{1}^{\prime}\left(S^{*}(z)\right) v_{1} \boldsymbol{K}_{0}(z) d z & =\boldsymbol{a}, \\
\lambda \Xi-\epsilon \int_{\mathbb{R}^{2}} \bar{\gamma}_{2}^{\prime}\left(S^{*}(z)\right) v_{1} k_{0}(z) d z & =T, \\
\lambda v_{0}+(\lambda-L) v_{1} & =g,
\end{aligned}
$$

where $v_{0}:=-q_{1} \partial_{z_{1}} S^{*}-q_{2} \partial_{z_{2}} S^{*}-\Xi \partial_{R} S^{*}$. Since $\rho_{\epsilon} \subset \rho(L)$, equation (2.10) is solvable for $v_{1}$ as $v_{1}=(\lambda-L)^{-1} g-\lambda(\lambda-L)^{-1} v_{0}$ and the estimate $\left\|v_{1}\right\|_{L^{2}} \leq c_{2}^{\prime \prime}\left\{\|g\|_{L^{2}}+|\lambda|(|\boldsymbol{q}|+|\Xi|)\right\}$ holds for $c_{2}^{\prime \prime}>0$. Therefore, by taking $c_{2}^{\prime}>0$ large enough, equations (2.8) and (2.9) are solvable for $\boldsymbol{q}$ and $\Xi$, that is, $\lambda \in \rho\left(\bar{L}_{0}\right)$.

Next, we show $\lambda \neq 0$ with $|\lambda| \leq c_{2}^{\prime} \epsilon$ should be $\lambda \in \rho\left(\bar{L}_{0}\right)$. Let $\lambda=\epsilon \lambda_{1}$ with $\lambda_{1} \neq 0$ and $\left|\lambda_{1}\right| \leq c_{2}^{\prime}$. We again consider equation (2.6) and get (2.8) $-(2.10)$ and $v_{1}$, too. Since $\left(\epsilon \lambda_{1}-L\right)^{-1}$ exists, (2.8) and (2.9) give the equation

$$
\left\{\begin{array}{l}
\epsilon \lambda_{1} \boldsymbol{q}+\epsilon^{2}\left\{q_{1} \boldsymbol{a}_{11}+q_{2} \boldsymbol{a}_{12}+\Xi \boldsymbol{a}_{13}\right\}=\boldsymbol{a}+\epsilon \boldsymbol{a}_{14}(g), \\
\epsilon \lambda_{1} \Xi+\epsilon^{2}\left\{q_{1} a_{21}+q_{2} a_{22}+\Xi a_{23}\right\}=T+\epsilon a_{24}(g),
\end{array}\right.
$$


where

$$
\begin{aligned}
\boldsymbol{a}_{1 j} & :=-\lambda_{1} \int_{\mathbb{R}^{2}} \bar{\gamma}_{1}^{\prime}\left(S^{*}(\boldsymbol{z})\right)\left\{\left(\epsilon \lambda_{1}-L\right)^{-1} \partial_{z_{j}} S^{*}\right\}(z) \boldsymbol{K}_{0}(z) d z, \quad(j=1,2) \\
\boldsymbol{a}_{13} & :=-\lambda_{1} \int_{\mathbb{R}^{2}} \bar{\gamma}_{1}^{\prime}\left(S^{*}(z)\right)\left\{\left(\epsilon \lambda_{1}-L\right)^{-1} \partial_{R} S^{*}\right\}(z) \boldsymbol{K}_{0}(z) d z \\
a_{2 j} & :=-\lambda_{1} \int_{\mathbb{R}^{2}} \bar{\gamma}_{2}^{\prime}\left(S^{*}(z)\right)\left\{\left(\epsilon \lambda_{1}-L\right)^{-1} \partial_{z_{j}} S^{*}\right\}(z) k_{0}(z) d z, \quad(j=1,2) \\
a_{23}:= & -\lambda_{1} \int_{\mathbb{R}^{2}} \bar{\gamma}_{2}^{\prime}\left(S^{*}(\boldsymbol{z})\right)\left\{\left(\epsilon \lambda_{1}-L\right)^{-1} \partial_{R} S^{*}\right\}(z) k_{0}(z) d z \\
\boldsymbol{a}_{14}:= & \int_{\mathbb{R}^{2}} \bar{\gamma}_{1}^{\prime}\left(S^{*}(z)\right)\left\{\left(\epsilon \lambda_{1}-L\right)^{-1} g\right\}(z) \boldsymbol{K}_{0}(z) d z \\
a_{24}:= & \int_{\mathbb{R}^{2}} \bar{\gamma}_{2}^{\prime}\left(S^{*}(z)\right)\left\{\left(\epsilon \lambda_{1}-L\right)^{-1} g\right\}(z) k_{0}(z) d z .
\end{aligned}
$$

Since $\left(\epsilon \lambda_{1}-L\right)^{-1}$ is bounded, equation (2.11) is solvable for $\boldsymbol{q}$ and $\Xi$ for sufficiently small $\epsilon>0$, which means $\lambda=\epsilon \lambda_{1} \in \rho\left(\bar{L}_{0}\right)$ except 0 in an $O(\epsilon)$ - neighborhood of the origin.

Finally, the non-degeneracy (semi-simpleness) of 0 eigenvalue and $\operatorname{Ker} \bar{L}_{0}=\operatorname{span}\left\{\bar{\Phi}_{1}, \bar{\Phi}_{2}, \bar{\Phi}_{3}\right\}$ are shown as follows. Let $\bar{L}_{0} \bar{V}=0$ with $\bar{V}={ }^{t}(\boldsymbol{q}, \Xi, v)$. Similarly to (2.6), $\bar{L}_{0} \bar{V}_{1}=0$ holds by taking $\bar{V}=\bar{V}_{0}+\bar{V}_{1}$ with $\bar{V}_{0}=q_{1} \bar{\Phi}_{1}+q_{2} \bar{\Phi}_{2}+\Xi \bar{\Phi}_{3}$ and $\bar{V}_{1}={ }^{t}\left(\mathbf{0}, 0, v_{1}\right)$. Then (2.10) with $\lambda=0$ gives $v_{1}=0$, which means $\bar{V}_{1}=0$ and therefore $\bar{V}=\bar{V}_{0}$. Thus, $\operatorname{Ker} \bar{L}_{0}=\operatorname{span}\left\{\bar{\Phi}_{1}, \bar{\Phi}_{2}, \bar{\Phi}_{3}\right\}$ was shown.

Assume $-\bar{L}_{0} \bar{V}=\bar{\Phi}_{1}$, where $\bar{V}={ }^{t}(\boldsymbol{q}, \Xi, v)$. Then we can similarly take $\bar{V}=\bar{V}_{0}+\bar{V}_{1}$ with $\bar{V}_{0}=q_{1} \bar{\Phi}_{1}+q_{2} \bar{\Phi}_{2}+\Xi \bar{\Phi}_{3}, \bar{V}_{1}={ }^{t}\left(\mathbf{0}, 0, v_{1}\right)$ and similarly to (2.8) - (2.10) also obtain

$$
\begin{aligned}
-\epsilon \int_{\mathbb{R}^{2}} \bar{\gamma}_{1}^{\prime}\left(S^{*}(z)\right) v_{1} \boldsymbol{K}_{0}(z) d z & =\boldsymbol{e}_{1}, \\
-\epsilon \int_{\mathbb{R}^{2}} \bar{\gamma}_{2}^{\prime}\left(S^{*}(\boldsymbol{z})\right) v_{1} k_{0}(z) d z & =0 \\
-L v_{1} & =\partial_{z_{1}} S^{*} .
\end{aligned}
$$

Thus, $v_{1}=-L^{-1} \partial_{z_{1}} S^{*}$ from (2.14) contradicts (2.12) and (2.13). Consequently, it is shown that there are no $\bar{V}$ satisfying $\bar{L}_{0} \bar{V}=\bar{\Phi}_{j}(j=1,2,3)$. Thus, the semi-simpleness of the 0 eigenvalue is proved.

Lemma 2.1 directly leads to the following theorem.

Theorem 2.1. If $\gamma_{1}$ and $\gamma_{2}$ are sufficiently small, then $S(\boldsymbol{x} ; \boldsymbol{p}, \Theta)$ is asymptotically stable except for translation and rotation. That is, if the initial data $\left(\boldsymbol{p}_{0}, \Theta_{0}, u_{0}(\boldsymbol{x})\right)$ is sufficiently close to $\left(\boldsymbol{p}_{0}, \Theta_{0}, S\left(\boldsymbol{x} ; \boldsymbol{p}_{0}, \Theta_{0}\right)\right)$, then there exists $\left(\boldsymbol{p}^{*}, \Theta^{*}\right)$ close to $\left(\boldsymbol{p}_{0}, \Theta_{0}\right)$ such that the solution $(\boldsymbol{p}(t), \Theta(t), u(t, \boldsymbol{x}))$ of (2.4) converges to $\left(\boldsymbol{p}^{*}, \Theta^{*}, S\left(\boldsymbol{x} ; \boldsymbol{p}^{*}, \Theta^{*}\right)\right)$ as $t \rightarrow \infty$.

Remark 2.1. When the linearized operator $\bar{L}_{0}$ satisfies the properties stated in Lemma 2.1, that $i$, the spectral set $\sigma\left(\bar{L}_{0}\right)$ is given by $\sigma\left(\bar{L}_{0}\right)=\{0\} \cup \sigma_{1}$ with $\sigma_{1} \subset\left\{\lambda \in \mathbb{C} ; \operatorname{Re}(\lambda)<-c_{2}\right\}$ for $c_{2}>0$ and 0 is a semi-simple eigenvalue, we call $S^{*}($ also $\bar{S}(\boldsymbol{x} ; \mathbf{0}, 0))$ linearly stable. Note that if $S^{*}$ is linearly stable, then $S(\boldsymbol{x} ; \boldsymbol{p}, \Theta)$ is also linearly stable for any $\boldsymbol{p} \in \mathbb{R}^{2}$ and $\Theta \in \mathbb{R}$. 
Remark 2.2. Here $\bar{L}(\boldsymbol{p}, \Theta)$ is given by

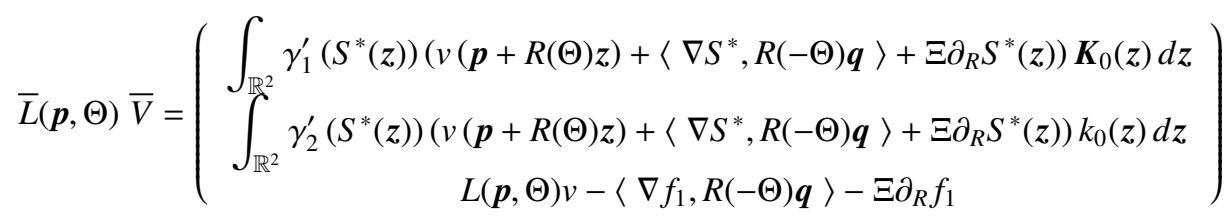

for $\bar{V}={ }^{t}(\boldsymbol{q}, \Xi, v) \in \bar{X}$, where $L(\boldsymbol{p}, \Theta):=d \Delta+f_{0}^{\prime}(S(\boldsymbol{x} ; \boldsymbol{p}, \Theta))$.

Proposition 2.2. We have

$$
\bar{\Phi}_{1}(\boldsymbol{p}, \Theta):=\left(\begin{array}{c}
R(\Theta) \boldsymbol{e}_{1} \\
0 \\
-\partial_{z_{1}} S^{*}\left(z^{*}\right)
\end{array}\right), \quad \bar{\Phi}_{2}(\boldsymbol{p}, \Theta):=\left(\begin{array}{c}
R(\Theta) \boldsymbol{e}_{2} \\
0 \\
-\partial_{z_{2}} S^{*}\left(z^{*}\right)
\end{array}\right), \quad \bar{\Phi}_{3}(\boldsymbol{p}, \Theta):=\left(\begin{array}{c}
\mathbf{0} \\
1 \\
-\partial_{R} S^{*}\left(z^{*}\right)
\end{array}\right)
$$

are the eigenfunctions of $\bar{L}(\boldsymbol{p}, \Theta)$ associated with a 0 eigenvalue for $z^{*}:=R(-\Theta)(\boldsymbol{x}-\boldsymbol{p})$.

Proof. By the relation between $\bar{L}_{0}$ and $\bar{L}(\boldsymbol{p}, \Theta)$, this can easily be verified.

Here, we remark about the adjoint operator $\bar{L}_{0}^{*}$ of $\bar{L}_{0}$ with respect to the inner product in the space $\bar{X}$, which is useful in investigating the interaction between two camphor particles in the next section.

Proposition 2.3. The adjoint operator $\bar{L}_{0}^{*}$ is

$$
{\overline{L_{0}}}^{*} \bar{V}=\left(\begin{array}{l}
\int_{\mathbb{R}^{2}}\left[\left\langle\boldsymbol{q}, \boldsymbol{K}_{0}(\boldsymbol{z})\right\rangle \gamma_{1}^{\prime}\left(S^{*}(\boldsymbol{z})\right) \nabla S^{*}+\Xi k_{0}(\boldsymbol{z}) \gamma_{2}^{\prime}\left(S^{*}(z)\right) \nabla S^{*}-v \nabla f_{1}\right] d z \\
\int_{\mathbb{R}^{2}}\left[\left\langle\boldsymbol{q}, \boldsymbol{K}_{0}(\boldsymbol{z})\right\rangle \gamma_{1}^{\prime}\left(S^{*}(\boldsymbol{z})\right) \partial_{R} S^{*}+\Xi k_{0}(\boldsymbol{z}) \gamma_{2}^{\prime}\left(S^{*}(z)\right) \partial_{R} S^{*}-v \partial_{R} f_{1}\right] d z \\
L v+\left\langle\boldsymbol{q}, \boldsymbol{K}_{0}(\boldsymbol{x})\right\rangle \gamma_{1}^{\prime}\left(S^{*}(\boldsymbol{x})\right)+\Xi k_{0}(\boldsymbol{x}) \gamma_{2}^{\prime}\left(S^{*}(\boldsymbol{x})\right)
\end{array}\right)
$$

for $\bar{V}={ }^{t}(\boldsymbol{q}, \Xi, v) \in \bar{X}$. The eigenfunctions associated with the 0 eigenvalue are given by $\bar{\Phi}_{j}^{*}:=$ $\left(\begin{array}{c}\boldsymbol{e}_{j} \\ 0 \\ \Phi_{j}^{*}\end{array}\right)(j=1,2)$ and $\bar{\Phi}_{3}^{*}:=\left(\begin{array}{c}\mathbf{0} \\ 1 \\ \Phi_{3}^{*}\end{array}\right)$, where $\Phi_{j}^{*}$ are the solutions satisfying

$$
\left\{\begin{array}{l}
L \Phi_{j}^{*}+\left\langle\boldsymbol{K}_{0}(\boldsymbol{x}), \boldsymbol{e}_{j}\right\rangle \gamma_{1}^{\prime}\left(S^{*}(\boldsymbol{x})\right)=0 \quad(j=1,2) \\
L \Phi_{3}^{*}+k_{0}(\boldsymbol{x}) \gamma_{2}^{\prime}\left(S^{*}(\boldsymbol{x})\right)=0
\end{array}\right.
$$

Proof. Here $\bar{L}_{0}^{*}$ is obtained by direct calculation according to the definition of an adjoint operator. We show that $\bar{\Phi}_{j}^{*}$ satisfies $\bar{L}_{0}^{*} \bar{\Phi}_{j}^{*}=0$ though we show it only for $\bar{\Phi}_{1}^{*}$.

Substituting $\bar{\Phi}_{1}^{*}$ into $\bar{L}_{0}^{*}$, we have

$$
\bar{L}_{0}^{*} \bar{\Phi}_{1}^{*}=\left(\begin{array}{c}
\int_{\mathbb{R}^{2}}\left[\left\langle\boldsymbol{e}_{1}, \boldsymbol{K}_{0}(\boldsymbol{z})\right\rangle \gamma_{1}^{\prime}\left(S^{*}(\boldsymbol{z})\right) \nabla S^{*}-\Phi_{1}^{*} \nabla f_{1}\right] d z \\
\int_{\mathbb{R}^{2}}\left[\left\langle\boldsymbol{e}_{1}, \boldsymbol{K}_{0}(\boldsymbol{z})\right\rangle \gamma_{1}^{\prime}\left(S^{*}(\boldsymbol{z})\right)-\Phi_{1}^{*} \partial_{R} f_{1}\right] d z \\
L \Phi_{1}^{*}+\left\langle\boldsymbol{e}_{1}, \boldsymbol{K}_{0}(\boldsymbol{x})\right\rangle \gamma_{1}^{\prime}\left(S^{*}(\boldsymbol{x})\right) \\
8
\end{array}\right) .
$$


The third component of (2.16) is 0 by the definition of $\Phi_{1}^{*}$. Now, we consider the first component of (2.16). Since the equation of $S^{*}$ in (2.5) holds, it follows that $L \partial_{x_{j}} S^{*}+\left\langle\nabla f_{1}, \boldsymbol{e}_{j}\right\rangle=0(j=1,2)$ by taking the derivative with respect to $x_{j}$ variable for $\boldsymbol{x}=\left(x_{1}, x_{2}\right)$. Then the $L^{2}$ - inner product with $\Phi_{1}^{*}$ leads to

$$
\left\{\begin{array}{l}
\left\langle\partial_{z_{1}} S^{*},\left\langle\boldsymbol{K}_{0}, \boldsymbol{e}_{1}\right\rangle \gamma_{1}^{\prime}\left(S^{*}\right)\right\rangle_{L^{2}}-\left\langle\left\langle\nabla f_{1}, \boldsymbol{e}_{1}\right\rangle, \Phi_{1}^{*}\right\rangle_{L^{2}}=0 \\
\left\langle\partial_{z_{2}} S^{*},\left\langle\boldsymbol{K}_{0}, \boldsymbol{e}_{1}\right\rangle \gamma_{1}^{\prime}\left(S^{*}\right)\right\rangle_{L^{2}}-\left\langle\left\langle\nabla f_{1}, \boldsymbol{e}_{2}\right\rangle, \Phi_{1}^{*}\right\rangle_{L^{2}}=0
\end{array}\right.
$$

by noting

$$
\left\langle L \partial_{z_{j}} S^{*}, \Phi_{1}^{*}\right\rangle_{L^{2}}=\left\langle\partial_{z_{j}} S^{*}, L \Phi_{1}^{*}\right\rangle_{L^{2}}=-\left\langle\partial_{z_{j}} S^{*},\left\langle\boldsymbol{K}_{0}, \boldsymbol{e}_{1}\right\rangle \gamma_{1}^{\prime}\left(S^{*}\right)\right\rangle_{L^{2}}
$$

This directly implies that the first component of (2.16) is 0 .

The second component of (2.16) is computed as follows: Taking the angle derivative $\partial_{R}=$ $-z_{2} \partial_{z_{1}}+z_{1} \partial_{z_{2}}$ in the equation of $S^{*}$ in (2.5), we have $L \partial_{R} S^{*}=\partial_{R} f_{1}$. By taking the $L^{2}$-inner product with $\Phi_{1}^{*}$ and using the definition of $\Phi_{1}^{*}$ again, we evaluate the second component of (2.16) as 0 .

Remark 2.3. The adjoint operator $\bar{L}^{*}(p, \Theta)$ is given by

$$
\bar{L}^{*}(\boldsymbol{p}, \Theta) \bar{V}=\left(\begin{array}{l}
R(\Theta) \int_{\mathbb{R}^{2}}\left[\left\langle\boldsymbol{q}, \boldsymbol{K}_{0}\right\rangle \gamma_{1}^{\prime}\left(S^{*}\right) \nabla S^{*}+\Xi k_{0} \gamma_{2}^{\prime}\left(S^{*}\right) \nabla S^{*}-v(\boldsymbol{p}+R(\Theta) \boldsymbol{z}) \nabla f_{1}\right] d \boldsymbol{z} \\
\int_{\mathbb{R}^{2}}\left[\left\langle\boldsymbol{q}, \boldsymbol{K}_{0}\right\rangle \gamma_{1}^{\prime}\left(S^{*}\right) \partial_{R} S^{*}+\Xi k_{0} \gamma_{2}^{\prime}\left(S^{*}\right) \partial_{R} S^{*}-v(\boldsymbol{p}+R(\Theta) \boldsymbol{z}) \partial_{R} f_{1}\right] d \boldsymbol{z} \\
L(\boldsymbol{p}, \Theta) v+\left\langle\boldsymbol{q}, \boldsymbol{K}_{0}\left(z^{*}\right)\right\rangle \gamma_{1}^{\prime}\left(S^{*}\left(z^{*}\right)\right)+\Xi k_{0}\left(z^{*}\right) \gamma_{2}^{\prime}\left(S^{*}\left(\boldsymbol{z}^{*}\right)\right)
\end{array}\right)
$$

for $\bar{V}={ }^{t}(\boldsymbol{q}, \Xi, v) \in \bar{X}$, where $z^{*}:=R(-\Theta)(\boldsymbol{x}-\boldsymbol{p})$. Here $\bar{\Phi}_{j}^{*}(\boldsymbol{p}, \Theta)(\boldsymbol{x}):=\bar{\Phi}_{j}^{*}\left(z^{*}\right)(j=1,2,3)$ are the eigenfunctions associated to the 0 eigenvalue of $\bar{L}^{*}(p, \Theta)$.

In the remainder of this section, we consider a stationary solution $S^{*}(\boldsymbol{x})$ in the case when the camphor particle $\Omega_{0}$ is slightly deformed from a disk to obtain precise information.

Let $B_{0}:=\left\{x \in \mathbb{R}^{2} ;|x|<r_{0}\right\}$ for $r_{0}>0$ and $C_{0}:=\partial B_{0}$. Introducing a small parameter $\varepsilon>0$, we give $\Omega_{0}$ as the inside of the boundary $\partial \Omega_{0}=\left\{\boldsymbol{x}=\left(r_{0}+\varepsilon b(\theta)\right) \boldsymbol{e}(\theta) ; 0 \leq \theta \leq 2 \pi\right\}$, where $b$ is a sufficiently smooth function and $\boldsymbol{e}(\theta):={ }^{t}(\cos \theta, \sin \theta)$.

To obtain asymptotic expansions of $S^{*}(\boldsymbol{x})$ with respect to $\varepsilon$, we first need to know the expansions of $\boldsymbol{K}_{0}(\boldsymbol{x}), k_{0}(\boldsymbol{x})$, and $f_{1}(\boldsymbol{x})$. Let $\Gamma_{\varepsilon}:=\partial \Omega_{0}$ and introduce the tubular coordinate $(s, \lambda)$ in the neighborhood of $\Gamma_{\varepsilon}$ by $\boldsymbol{x}=\boldsymbol{\Gamma}_{\varepsilon}(s)+\lambda \boldsymbol{n}_{\varepsilon}(s)$ for $\Gamma_{\varepsilon}=\left\{\boldsymbol{\Gamma}_{\varepsilon}(s)\right\}$ with the arc length parameter $s$ and the outward normal unit vector $\boldsymbol{n}_{\varepsilon}$ of $\Gamma_{\varepsilon}$. We also define functions $\Lambda_{\varepsilon}(\boldsymbol{x})$ and $\Sigma_{\varepsilon}(\boldsymbol{x})$ by $s=\Sigma_{\varepsilon}(\boldsymbol{x})$ and $\lambda=\Lambda_{\varepsilon}(\boldsymbol{x})$. Here $\Lambda_{\varepsilon}(\boldsymbol{x})$ is the signed distance function between $\boldsymbol{x}$ and $\Gamma_{\varepsilon}$ for $\boldsymbol{x}$ in the neighborhood of $\Gamma_{\varepsilon}$. The sign is defined by \pm for $\boldsymbol{x} \in \Omega_{0}^{c}$ and $\boldsymbol{x} \in \Omega_{0}$. Here we give the precise expressions of $\boldsymbol{K}_{0}(\boldsymbol{x}), k_{0}(\boldsymbol{x})$, and $f_{1}(\boldsymbol{x})$. Suppose that $\delta$ is a sufficiently small fixed positive number and that the supports of $\boldsymbol{K}_{0}(\boldsymbol{x})$ and $k_{0}(\boldsymbol{x})$ are included in the $\delta$-neighborhood of $\Gamma_{\varepsilon}$. Let $\xi(\lambda)$ be a smooth nonnegative function approximating the Dirac $\delta$-function of the order of $O(\delta)$ with $\operatorname{supp}(\xi) \subset(-\delta, \delta)$ and $\int_{\mathbb{R}} \xi(\lambda) d \lambda=1$. In fact, $\left|\int_{\mathbb{R}} g(\lambda) \xi(\lambda) d \lambda-g(0)\right| \leq \delta\|g\|_{H^{1}(\mathbb{R})}$ holds for $g \in C_{0}^{\infty}(\mathbb{R})$. Similarly, we let $\mu(\lambda)$ be a smooth monotone decreasing function approximating the Heaviside function of the order of $O(\delta)$ with the support in $(-\infty, \delta)$ and connecting 0 and 1 , that is, $\mu(\lambda) \equiv 0$ for $\lambda \geq \delta$ and $\mu(\lambda) \equiv 1$ for $\lambda<-\delta$. We may use $\xi(\lambda)=-\mu^{\prime}(\lambda)$ and, for 
simplicity, we assume it hereafter. Then $f_{1}(\boldsymbol{x})$ may be defined in the $\delta$-neighborhood of $\Gamma_{\varepsilon}$ by $f_{1}(\boldsymbol{x})=a_{0} \mu\left(\Lambda_{\varepsilon}(\boldsymbol{x})\right)$, further inside of $\Omega_{0}$ by $f_{1}(\boldsymbol{x}) \equiv a_{0}$, and further outside of $\Omega_{0}$ by $f_{1}(\boldsymbol{x}) \equiv 0$.

Since the integral $\int_{\mathbb{R}^{2}} g(\boldsymbol{x}) k_{0}(\boldsymbol{x}) d \boldsymbol{x}=\int_{\Gamma_{\varepsilon}} \int_{-\delta}^{\delta} g(\boldsymbol{x}) k_{0}(\boldsymbol{x})\left(1+\lambda \kappa_{\varepsilon}\right) d s d \lambda$ holds for a function $g$ with $\boldsymbol{x}=\boldsymbol{\Gamma}_{\varepsilon}(s)+\lambda \boldsymbol{n}_{\varepsilon}(s)$ and the curvature $\kappa_{\varepsilon}$ of $\Gamma_{\varepsilon}$, we can approximate the boundary integral $\int_{\Gamma_{\varepsilon}} g(\boldsymbol{x})\left(\boldsymbol{x} \times \boldsymbol{n}_{\varepsilon}\right) d s$ by $\int_{\mathbb{R}^{2}} g(\boldsymbol{x}) k_{0}(\boldsymbol{x}) d \boldsymbol{x}$ by giving $k_{0}(\boldsymbol{x})=\left(\boldsymbol{\Gamma}_{\varepsilon}\left(\Sigma_{\varepsilon}(\boldsymbol{x})\right) \times \boldsymbol{n}_{\varepsilon}\left(\Sigma_{\varepsilon}(\boldsymbol{x})\right)\right) \xi\left(\Lambda_{\varepsilon}(\boldsymbol{x})\right)$. Similarly, $\boldsymbol{K}_{0}(\boldsymbol{x})=\xi\left(\Lambda_{\varepsilon}(\boldsymbol{x})\right) \boldsymbol{n}_{\varepsilon}\left(\Sigma_{\varepsilon}(\boldsymbol{x})\right)$ is approximating the boundary integral $\int_{\Gamma_{\varepsilon}} g(\boldsymbol{x}) \boldsymbol{n}_{\varepsilon} d s$ by $\int_{\mathbb{R}^{2}} g(\boldsymbol{x}) \boldsymbol{K}_{0}(\boldsymbol{x}) d \boldsymbol{x}$.

Proposition 2.4. For $\boldsymbol{x}$ in the neighborhood of $\Gamma_{\varepsilon}, \Lambda_{\varepsilon}(\boldsymbol{x})=|\boldsymbol{x}|-r_{0}-\varepsilon b+O\left(\varepsilon^{2}\right)$ holds.

Proof. Since $\Gamma_{\varepsilon}$ is parametrized by

$$
\begin{gathered}
\boldsymbol{\Gamma}_{\varepsilon}(\theta)=\left(r_{0}+\varepsilon b(\theta)\right) \boldsymbol{e}(\theta), \\
\partial_{\theta} \boldsymbol{\Gamma}_{\varepsilon}(\theta)=\left(r_{0}+\varepsilon b(\theta)\right) \boldsymbol{e}^{\perp}(\theta)+\varepsilon b^{\prime}(\theta) \boldsymbol{e}(\theta)
\end{gathered}
$$

holds, where $\boldsymbol{e}^{\perp}(\theta):={ }^{t}(-\sin \theta, \cos \theta) \perp \boldsymbol{e}(\theta)$. Then the unit normal vector $\boldsymbol{n}_{\varepsilon}$ of $\Gamma_{\varepsilon}$ is given by

$$
\boldsymbol{n}_{\varepsilon}=-\frac{\varepsilon b^{\prime}}{\sqrt{\left(r_{0}+\varepsilon b\right)^{2}+\varepsilon^{2}\left(b^{\prime}\right)^{2}}} \boldsymbol{e}^{\perp}+\frac{r_{0}+\varepsilon b}{\sqrt{\left(r_{0}+\varepsilon b\right)^{2}+\varepsilon^{2}\left(b^{\prime}\right)^{2}}} \boldsymbol{e} .
$$

Here $\boldsymbol{x}=\boldsymbol{\Gamma}_{\varepsilon}(\theta)+\lambda \boldsymbol{n}_{\varepsilon}(\theta)$ and (2.17) imply

$$
\begin{aligned}
|\boldsymbol{x}|^{2} & =\left(r_{0}+\varepsilon b+\frac{\lambda\left(r_{0}+\varepsilon b\right)}{\sqrt{\left(r_{0}+\varepsilon b\right)^{2}+\varepsilon^{2}\left(b^{\prime}\right)^{2}}}\right)^{2}+\left(\frac{\varepsilon \lambda b^{\prime}}{\sqrt{\left(r_{0}+\varepsilon b\right)^{2}+\varepsilon^{2}\left(b^{\prime}\right)^{2}}}\right)^{2} \\
& =\left(\lambda+r_{0}+\varepsilon b\right)^{2}+O\left(\varepsilon^{2}\right),
\end{aligned}
$$

which leads the result.

By Proposition 2.4, we have

Proposition 2.5. For $x=r e(\theta)$,

$$
\begin{aligned}
f_{1}(\boldsymbol{x}) & =a_{0} \mu\left(r-r_{0}\right)-\varepsilon a_{0} b(\theta) \mu^{\prime}\left(r-r_{0}\right)+O\left(\varepsilon^{2}\right), \\
\boldsymbol{K}_{0}(\boldsymbol{x}) & =\xi\left(r-r_{0}\right) \boldsymbol{e}(\theta)-\varepsilon\left\{b(\theta) \xi^{\prime}\left(r-r_{0}\right) \boldsymbol{e}(\theta)+\frac{b^{\prime}(\theta) \xi\left(r-r_{0}\right)}{r_{0}} \boldsymbol{e}^{\perp}(\theta)\right\}+O\left(\varepsilon^{2}\right), \\
k_{0}(\boldsymbol{x}) & =-\varepsilon b^{\prime}(\theta) \xi\left(r-r_{0}\right)+O\left(\varepsilon^{2}\right)
\end{aligned}
$$

hold.

Proof. Substituting the result of Proposition 2.4 into the definition of $f_{1}$, we obtain the result.

For $\boldsymbol{K}_{0}, \boldsymbol{n}_{\varepsilon}=\boldsymbol{e}-\varepsilon b^{\prime} \boldsymbol{e}^{\perp}+O\left(\varepsilon^{2}\right)$ by (2.18) and Proposition 2.1 leads to the result.

Finally, the direct calculation by (2.17) and (2.18) gives $\boldsymbol{\Gamma}_{\varepsilon} \times \boldsymbol{n}_{\varepsilon}=-\varepsilon b^{\prime}+O\left(\varepsilon^{3}\right)$. Then by Proposition 2.1, we have $k_{0}(\boldsymbol{x})=\left(-\varepsilon b^{\prime}(\theta)+O\left(\varepsilon^{3}\right)\right) \xi\left(r-r_{0}+O(\varepsilon)\right)=-\varepsilon b^{\prime}(\theta) \xi\left(r-r_{0}\right)+O\left(\varepsilon^{2}\right)$. 
Now, we show an asymptotic expansion of the stationary solution $S^{*}$ satisfying (2.5) on slightly deformed domain $\Omega_{0}$ from a disk. Let $S^{*}=S_{0}^{*}+\varepsilon S_{1}^{*}+\cdots$. Then the third equation of (2.5) gives the equation of $S_{0}^{*}$

$$
0=d \Delta S_{0}^{*}+f_{0}\left(S_{0}^{*}\right)+a_{0} \mu\left(r-r_{0}\right)
$$

for $r=|\boldsymbol{x}|$. In the typical case of $f_{0}(u)=-\alpha u$, this is solved. Hence, we make the following assumption:

(H3) Equation (2.19) is uniquely solved by $S_{0}^{*}(\boldsymbol{x})=U_{0}(r)>0$ as a radially symmetric function and the linearized operator $L_{0}:=d \Delta+f_{0}^{\prime}\left(U_{0}(r)\right)$ has the property $\sigma\left(L_{0}\right) \subset\left\{\lambda \in \mathbb{C} ; \operatorname{Re}(\lambda)<-c_{1}\right\}$.

Then by the implicit function theorem, the existence of the solution $S^{*}=S_{\varepsilon}^{*}$ satisfying the third equation of (2.5) is shown for sufficiently small $\varepsilon>0$.

Remark 2.4. The stationary solution of (2.4) exists when the disk shape has $D_{2 n}$ symmetry $(2 n-$ fold rotational symmetry and chiral symmetry), where $n \in \mathbb{N}$. When $\gamma_{1}(u)=\gamma(u) / \tau_{1} \equiv 0$, i.e., the translation is prohibited due to the infinitely large resistance $\left(\tau_{1} \rightarrow \infty\right)$, the stationary solution exists when the disk shape has $D_{n}$ symmetry.

In actual systems, slight asymmetry is inevitable in the process of constructing experimental apparatuses. Even though such asymmetry exists, the torque arising from it can balance static friction, e.g., the friction between the particles and shafts in our experimental system.

Hence, we restrict our investigation only on the case of $b(\theta)=\cos m_{0} \theta$ hereafter. Let $A_{j}:=$ $d\left(\partial_{r}^{2}+\frac{1}{r} \partial_{r}-\frac{j^{2}}{r^{2}}\right)+f_{0}^{\prime}\left(U_{0}(r)\right)(j=0,1,2, \ldots)$ and $X_{R}:=\left\{u ; \int_{0}^{\infty} r u^{2}(r) d r<+\infty\right\}$, the function space consisting of radially symmetric functions belonging to $L^{2}\left(\mathbb{R}^{2}\right)$. Note that $A_{m_{0}}$ is invertible in $X_{R}$ by the invertibility of $L_{0}$ in $L^{2}\left(\mathbb{R}^{2}\right)$. Then the equation of $S_{1}^{*}$ is

$$
0=L_{0} S_{1}^{*}-a_{0} b(\theta) \mu^{\prime}\left(r-r_{0}\right)=L_{0} S_{1}^{*}+a_{0} b(\theta) \xi\left(r-r_{0}\right)
$$

and $S_{1}^{*}$ is given by $S_{1}^{*}(r, \theta)=b(\theta) U_{1}(r)$, where $U_{1}(r) \in X_{R}$ is the unique solution of

$$
A_{m_{0}} U_{1}=-a_{0} \xi\left(r-r_{0}\right) \text {. }
$$

Thus, we have $S^{*}(r \boldsymbol{e}(\theta))=U_{0}(r)+\varepsilon b(\theta) U_{1}(r)+O\left(\varepsilon^{2}\right)$. By this expression of $S^{*}$, we easily obtain the following proposition.

Proposition 2.6. We have that $\bar{\Phi}_{1}=\left(\begin{array}{c}\boldsymbol{e}_{1} \\ 0 \\ -U_{0}^{\prime} \cos \theta\end{array}\right)+O(\varepsilon), \bar{\Phi}_{2}=\left(\begin{array}{c}\boldsymbol{e}_{2} \\ 0 \\ -U_{0}^{\prime} \sin \theta\end{array}\right)+O(\varepsilon)$, and $\bar{\Phi}_{3}=$ $\left(\begin{array}{c}\mathbf{0} \\ 1 \\ \varepsilon m_{0} U_{1}(r) \sin m_{0} \theta\end{array}\right)+O\left(\varepsilon^{2}\right)$ hold. In addition, $\bar{\Phi}_{1}^{*}=\left(\begin{array}{c}\boldsymbol{e}_{1} \\ 0 \\ U_{0}^{*} \cos \theta\end{array}\right)+O(\varepsilon), \bar{\Phi}_{2}^{*}=\left(\begin{array}{c}\boldsymbol{e}_{2} \\ 0 \\ U_{0}^{*} \sin \theta\end{array}\right)+$ $O(\varepsilon)$, and $\bar{\Phi}_{3}^{*}=\left(\begin{array}{c}\mathbf{0} \\ 1 \\ \varepsilon V_{1}^{*} \sin m_{0} \theta\end{array}\right)+O\left(\varepsilon^{2}\right)$ hold, where $U_{0}^{*}=U_{0}^{*}(r)$ and $V_{1}^{*}=V_{1}^{*}(r)$ are functions satisfying $A_{1} U_{0}^{*}+\xi\left(r-r_{0}\right) \gamma_{1}^{\prime}\left(U_{0}(r)\right)=0$ and $A_{m_{0}} V_{1}^{*}+m_{0} \xi\left(r-r_{0}\right) \gamma_{2}^{\prime}\left(U_{0}(r)\right)=0$, respectively. 

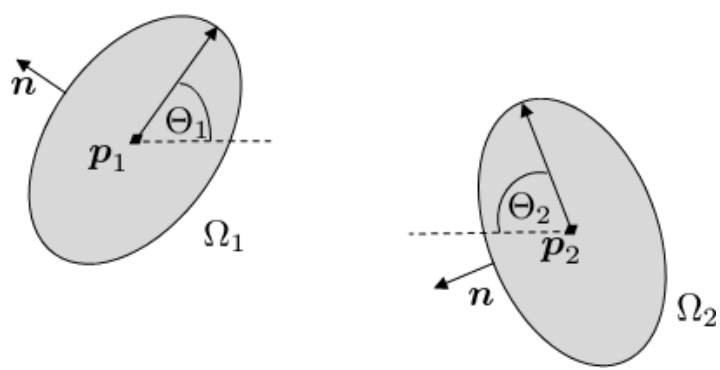

Figure 3: Two camphor particles.

Proof. Equation (2.15) and Proposition 2.5 directly give the proof.

In this section, we considered a camphor particle in an isolated situation, and discussed the stability of the rest state of a camphor particle at arbitrary position $\boldsymbol{p}$ and with arbitrary angle $\Theta$. We proved that for sufficiently small $\gamma_{1}$ and $\gamma_{2}$, the rest state is linearly stable except for translation and rotation, which correspond to neutral modes. Here, the condition with small $\gamma_{1}$ and $\gamma_{2}$ are realized for large resistance on translation and rotation.

As shown in Proposition 2.3, we explicitly obtained adjoint operator of the time evolution operator. By considering three neutral modes (two for translation and one for rotation), we obtained also adjoint vectors.

\section{Interaction of two camphor particles}

In this section, we consider two camphor particles with the same shapes as in Fig. 3, which is described by the equation

$$
\left\{\begin{aligned}
\dot{\boldsymbol{p}}_{1} & =\int_{\mathbb{R}^{2}} \gamma_{1}\left(u\left(\boldsymbol{p}_{1}+R_{1}\left(\Theta_{1}\right) \boldsymbol{z}\right)\right) \boldsymbol{K}_{0}(\boldsymbol{z}) d \boldsymbol{z} \\
\dot{\Theta}_{1} & =\int_{\mathbb{R}^{2}} \gamma_{2}\left(u\left(\boldsymbol{p}_{1}+R_{1}\left(\Theta_{1}\right) \boldsymbol{z}\right)\right) k_{0}(\boldsymbol{z}) d \boldsymbol{z} \\
\dot{\boldsymbol{p}}_{2} & =\int_{\mathbb{R}^{2}} \gamma_{1}\left(u\left(\boldsymbol{p}_{2}+R_{2}\left(\Theta_{2}\right) \boldsymbol{z}\right)\right) \boldsymbol{K}_{0}(\boldsymbol{z}) d \boldsymbol{z} \\
\dot{\Theta}_{2} & =\int_{\mathbb{R}^{2}} \gamma_{2}\left(u\left(\boldsymbol{p}_{2}+R_{2}\left(\Theta_{2}\right) \boldsymbol{z}\right)\right) k_{0}(\boldsymbol{z}) d \boldsymbol{z} \\
\partial_{t} u & =d \Delta u+f_{0}(u)+f_{1}\left(R_{1}\left(-\Theta_{1}\right)\left(\boldsymbol{x}-\boldsymbol{p}_{1}\right)\right)+f_{1}\left(R_{2}\left(-\Theta_{2}\right)\left(\boldsymbol{x}-\boldsymbol{p}_{2}\right)\right)
\end{aligned}\right.
$$

where $R_{1}(\Theta):=R(\Theta)$ and $R_{2}(\Theta):=R(\pi-\Theta)$.

First, we give the equation of motion describing two stable camphor particles in general situations. Suppose that there exists linearly stable stationary solution $S^{*}(z)$ satisfying (2.5) and use the same notions as in Section 2. That is, we make the following assumption:

(H4) The results of Lemma 2.1 hold for $S^{*}$ without the assumption on the smallness of $\gamma_{1}$ and $\gamma_{2}$. 
Define $z_{1}^{*}:=R_{1}\left(-\Theta_{1}\right)\left(\boldsymbol{x}-\boldsymbol{p}_{1}\right)$ and $z_{2}^{*}:=R_{2}\left(-\Theta_{2}\right)\left(\boldsymbol{x}-\boldsymbol{p}_{2}\right)$. Since $R_{1}^{\prime}(\Theta)=R(\pi / 2) R_{1}(\Theta)$ and $\partial_{t} g=-\left\langle R_{1}(-\Theta) \dot{\boldsymbol{p}}+\dot{\Theta} R(\pi / 2) z_{1}^{*}, \nabla g\right\rangle=-\left\langle R_{1}(-\Theta) \dot{p}, \nabla g\right\rangle-\dot{\Theta} \partial_{R_{1}} g=-B_{1} g$ hold for $g=g\left(z_{1}^{*}\right)$ in which $\nabla g$ means $\nabla_{z} g$ and $\partial_{R_{1}}:=\partial_{R}$, we define $B_{1}=B_{1}(\boldsymbol{p}, \Theta):=\left\langle R_{1}(-\Theta) \dot{p}, \nabla\right.$. \rangle$+\dot{\Theta} \partial_{R_{1}}$. Similarly, $R_{2}^{\prime}(\Theta)=R(-\pi / 2) R_{2}(\Theta)$ and $\partial_{t} g=-\left\langle R_{2}(-\Theta) \dot{p}+\dot{\Theta} R(-\pi / 2) z_{2}^{*}, \nabla g\right\rangle=$ $-\left\langle R_{2}(-\Theta) \dot{\boldsymbol{p}}, \nabla g\right\rangle-\dot{\Theta} \partial_{R_{2}} g=-B_{2}(\boldsymbol{p}, \Theta) g$ hold for $g=g\left(z_{2}^{*}\right)$, where $\partial_{R_{2}} g:=\langle R(-\pi / 2) z, \nabla g\rangle=$ $-\partial_{R_{1}} g$ and $B_{2}=B_{2}(\boldsymbol{p}, \Theta):=\left\langle R_{2}(-\Theta) \dot{p}, \nabla \cdot\right\rangle+\dot{\Theta} \partial_{R_{2}}$. Then, we have

$$
\begin{aligned}
\partial_{t} v= & L\left(S^{1}+S^{2}\right) v+B_{1} S^{1}+B_{2} S^{2} \\
& +f_{0}\left(S^{1}+S^{2}\right)-f_{0}\left(S^{1}\right)-f_{0}\left(S^{2}\right)+g_{0}(v)
\end{aligned}
$$

by putting $u(t, \boldsymbol{x})=S^{*}\left(z_{1}^{*}\right)+S^{*}\left(z_{2}^{*}\right)+v(t, \boldsymbol{x})$ with $\left|\boldsymbol{p}_{2}-\boldsymbol{p}_{1}\right| \gg 1$, where we use the notation $L(g):=d \Delta+f_{0}^{\prime}(g)$ for a function $g, S^{1}:=S^{*}\left(z_{1}^{*}\right), S^{2}:=S^{*}\left(z_{2}^{*}\right)$, and $g_{0}(v):=f_{0}\left(S^{1}+S^{2}+v\right)-$ $f_{0}\left(S^{1}+S^{2}\right)-f_{0}^{\prime}\left(S^{1}+S^{2}\right) v=O\left(v^{2}\right)$.

Let $\widetilde{X}:=\left(\mathbb{R}^{2} \times \mathbb{R}\right) \times\left(\mathbb{R}^{2} \times \mathbb{R}\right) \times L^{2}\left(\mathbb{R}^{2}\right), \widetilde{U}:={ }^{t}\left(\boldsymbol{p}_{1}, \Theta_{1}, \boldsymbol{p}_{2}, \Theta_{2}, u\right) \in \widetilde{X}$, and the right-hand side of (3.1) be $\widetilde{\mathcal{L}}(\widetilde{U})$. We also put $\widetilde{S^{1}}\left(\boldsymbol{x} ; \boldsymbol{p}_{1}, \Theta_{1}\right):={ }^{t}\left(\boldsymbol{p}_{1}, \Theta_{1}, \mathbf{0}, 0, S^{*}\left(z_{1}^{*}\right)\right), \widetilde{S^{2}}\left(\boldsymbol{x} ; \boldsymbol{p}_{2}, \Theta_{2}\right):=$ ${ }^{t}\left(\mathbf{0}, 0, \boldsymbol{p}_{2}, \Theta_{2}, S^{*}\left(z_{2}^{*}\right)\right)$, and use the notation $\widetilde{L}(\widetilde{U}):=\widetilde{\mathcal{L}^{\prime}}(\widetilde{U})$. Then by taking $\widetilde{U}=\widetilde{S^{1}}+\widetilde{S^{2}}+\widetilde{V}$ with $\widetilde{V}={ }^{t}\left(\boldsymbol{q}_{1}, \Xi_{1}, \boldsymbol{q}_{2}, \Xi_{2}, v(t, \boldsymbol{x})\right)$, we see from the equation (3.1) that $\partial_{t} \widetilde{U}=\widetilde{\mathcal{L}}(\widetilde{U})$ is

$$
\partial_{t} \widetilde{V}=\widetilde{L}\left(\widetilde{S^{1}}+\widetilde{S^{2}}\right) \widetilde{V}-\dot{\bar{y}}+\widetilde{B_{1}} \widetilde{S^{1}}+\widetilde{B_{2}} \widetilde{S^{2}}+\widetilde{\mathcal{L}}\left(\widetilde{S^{1}}+\widetilde{S^{2}}\right)+\widetilde{G_{1}},
$$

where $\widetilde{\boldsymbol{y}}={ }^{t}\left(\boldsymbol{p}_{1}, \Theta_{1}, \boldsymbol{p}_{2}, \Theta_{2}, 0\right), \widetilde{B_{1}} \widetilde{S^{1}}:={ }^{t}\left(\mathbf{0}, 0, \mathbf{0}, 0, B_{1} S^{1}\right)$, and $\widetilde{B_{2}} \widetilde{S^{2}}:={ }^{t}\left(\mathbf{0}, 0, \mathbf{0}, 0, B_{2} S^{2}\right)$. In addition, $\widetilde{G_{1}}:={ }^{t}\left(\boldsymbol{g}_{1}, g_{1}, \boldsymbol{g}_{2}, g_{2}, g_{3}\right)$ with $\boldsymbol{g}_{j}=\boldsymbol{g}_{j}\left(\boldsymbol{q}_{j}, \Xi_{j}, v\right)=O\left(\left|\boldsymbol{q}_{j}\right|^{2}+\left|\Xi_{j}\right|^{2}+|v|^{2}\right)(j=1,2)$ and $g_{j}=g_{j}\left(\boldsymbol{q}_{j}, \Xi_{j}, v\right)=O\left(\left|\boldsymbol{q}_{j}\right|^{2}+\left|\Xi_{j}\right|^{2}+|v|^{2}\right)(j=1,2,3)$.

Let $h:=\left|\boldsymbol{p}_{2}-\boldsymbol{p}_{1}\right|, \delta(h):=e^{-\alpha_{0} h}$ and define projections from $\widetilde{X}$ to $\bar{X}$ by $P_{1} \widetilde{U}:={ }^{t}\left(\boldsymbol{p}_{1}, \Theta_{1}, u\right)$, $P_{2} \widetilde{U}:={ }^{t}\left(\boldsymbol{p}_{2}, \Theta_{2}, u\right)$ for $\widetilde{U}:={ }^{t}\left(\boldsymbol{p}_{1}, \Theta_{1}, \boldsymbol{p}_{2}, \Theta_{2}, u\right) \in \widetilde{X}$. Define $\bar{L}_{1}(\boldsymbol{p}, \Theta):=\bar{L}(\boldsymbol{p}, \Theta), \bar{L}_{2}(\boldsymbol{p}, \Theta):=$ $\bar{L}(\boldsymbol{p}, \pi-\Theta)$, and $\widetilde{Y}:=\left\{\tilde{\boldsymbol{y}}=\left(\boldsymbol{p}_{1}, \Theta_{1}, \boldsymbol{p}_{2}, \Theta_{2}\right) \in \mathbb{R}^{6} ; 0 \leq \Theta_{j}<2 \pi,\left|\boldsymbol{p}_{2}-\boldsymbol{p}_{1}\right|>h^{*}\right\}$ for sufficiently large fixed $h^{*}>0$. Hereafter, we identify $\widetilde{\boldsymbol{y}}=\left(\boldsymbol{p}_{1}, \Theta_{1}, \boldsymbol{p}_{2}, \Theta_{2}\right) \in \mathbb{R}^{6}$ and $\widetilde{\boldsymbol{y}}=\left(\boldsymbol{p}_{1}, \Theta_{1}, \boldsymbol{p}_{2}, \Theta_{2}, 0\right) \in$ $\mathbb{R}^{6} \times L^{2}\left(\mathbb{R}^{2}\right)$ with the same notation and also put $\widetilde{\boldsymbol{y}}_{1}:=\left(\boldsymbol{p}_{1}, \Theta_{1}, \mathbf{0}, 0\right)$ and $\widetilde{\boldsymbol{y}}_{2}:=\left(\mathbf{0}, 0, \boldsymbol{p}_{2}, \Theta_{2}\right)$ for $\widetilde{\boldsymbol{y}}=\left(\boldsymbol{p}_{1}, \Theta_{1}, \boldsymbol{p}_{2}, \Theta_{2}\right)$. Assumptions (H1) and (H2) lead to the following proposition:

Proposition 3.1. For $h>h^{*}$, the spectral set $\sigma\left(\widetilde{L}_{S}(\widetilde{y})\right)=\sigma_{0}(\widetilde{y}) \cup \sigma_{1}(\widetilde{y})$ with $\sigma_{0}(\widetilde{\boldsymbol{y}}) \subset\left\{\lambda \in \mathbb{C} ;|\lambda|<O\left(\delta^{1 / 4}(h)\right)\right\}$ and $\sigma_{1}(\widetilde{\boldsymbol{y}}) \subset\left\{\lambda \in \mathbb{C} ; \operatorname{Re}(\lambda)<-c_{3}\right\}$ for $c_{3}>0$, where $\widetilde{\boldsymbol{y}}=\left(\boldsymbol{p}_{1}, \Theta_{1}, \boldsymbol{p}_{2}, \Theta_{2}\right) \in \widetilde{Y}$ and $\widetilde{L}_{S}(\widetilde{\boldsymbol{y}}):=$ $\widetilde{L}\left(\widetilde{S^{1}}+\widetilde{S^{2}}\right)$.

Proof. Let $\Omega_{1}:=\left\{\boldsymbol{x} \in \mathbb{R}^{2} ;\left|\boldsymbol{x}-\boldsymbol{p}_{1}\right|<\left|\boldsymbol{x}-\boldsymbol{p}_{2}\right|\right\}$ and $\Omega_{2}:=\left\{\boldsymbol{x} \in \mathbb{R}^{2} ;\left|\boldsymbol{x}-\boldsymbol{p}_{1}\right|>\left|\boldsymbol{x}-\boldsymbol{p}_{2}\right|\right\}$. Since

$$
P_{1} \widetilde{L}_{S}(\widetilde{\boldsymbol{y}}) \widetilde{W}-\bar{L}_{1}\left(\boldsymbol{p}_{1}, \Theta_{1}\right) P_{1} \widetilde{W}={ }^{t}\left(\mathbf{0}, 0,-\left\langle\nabla f_{1}\left(z_{2}^{*}\right), \boldsymbol{q}_{2}\right\rangle-\Xi_{2} \partial_{R} f_{1}\left(z_{2}^{*}\right)\right)+O(\delta(h))
$$

and

$$
P_{2} \widetilde{L}_{S}(\widetilde{\boldsymbol{y}}) \widetilde{W}-\bar{L}_{2}\left(\boldsymbol{p}_{2}, \Theta_{2}\right) P_{2} \widetilde{W}={ }^{t}\left(\mathbf{0}, 0,-\left\langle\nabla f_{1}\left(z_{1}^{*}\right), \boldsymbol{q}_{1}\right\rangle-\Xi_{1} \partial_{R} f_{1}\left(z_{1}^{*}\right)\right)+O(\delta(h))
$$

hold for $\widetilde{W}={ }^{t}\left(\boldsymbol{q}_{1}, \Xi_{1}, \boldsymbol{q}_{2}, \Xi_{2}, w\right) \in \widetilde{X}$, it follows that

$$
\left\|P_{j} \widetilde{L}_{S}(\widetilde{\boldsymbol{y}}) \widetilde{W}-\bar{L}_{j}\left(\boldsymbol{p}_{j}, \Theta_{j}\right) P_{j} \widetilde{W}\right\|_{L^{\infty}\left(\Omega_{j}\right)} \leq O(\delta(h))\|\widetilde{W}\|_{\infty} \quad(j=1,2)
$$

by the property of $f_{1}$, that is, $f_{1}\left(z_{j}^{*}\right)=0$ on $\Omega_{i}$ for $i \neq j$. Then we can show the results by applying the methods in [36] and [37] together with assumption (H3). 
Let $\widetilde{E}_{0}(\widetilde{y})$ and $\widetilde{E}_{1}(\widetilde{y})$ be the corresponding eigenspaces to the spectral sets $\sigma_{0}(\widetilde{y})$ and $\sigma_{1}(\widetilde{y})$, respectively, together with projections $\widetilde{Q}_{j}(\widetilde{y}): \widetilde{X} \rightarrow \widetilde{E}_{j}(\widetilde{y})$ for $j=0,1$ for $\widetilde{y}=\left(\boldsymbol{p}_{1}, \Theta_{1}, p_{2}, \Theta_{2}\right) \in$ $\widetilde{Y}$. Then in the similar manner to [36], we have

Proposition 3.2. $\left.\widetilde{E}_{0} \widetilde{\boldsymbol{y}}\right)$ is spanned by $\left\{\widetilde{\Phi}_{j}(\widetilde{y})\right\}(j=1, \ldots, 6)$ satisfying $P_{1} \widetilde{\Phi}_{j}(\widetilde{y})=\bar{\Phi}_{j}\left(p_{1}, \Theta_{1}\right)+$ $O(\delta(h)), P_{2} \widetilde{\Phi}_{j}(\widetilde{y})=O(\delta(h))(j=1,2,3)$ and $P_{1} \widetilde{\Phi}_{j}(\widetilde{y})=O(\delta(h)), P_{2} \widetilde{\Phi}_{j}(\widetilde{y})=\bar{\Phi}_{j}\left(\boldsymbol{p}_{2}, \pi-\Theta_{2}\right)+$ $O(\delta(h))(j=4,5,6)$.

For the adjoint operator $\left.\widetilde{L_{S}^{*}} \widetilde{\boldsymbol{y}}\right):=\widetilde{L}^{*}\left(\widetilde{S^{1}}+\widetilde{S^{2}}\right)$, quite similar properties to $\widetilde{L}_{S}(\widetilde{y})$ hold. That is, we have the following result.

Proposition 3.3. For $h>h^{*}$, the spectral set $\sigma\left(\widetilde{L}_{S}^{*}(\widetilde{y})\right)=\sigma_{0}^{*}(\widetilde{y}) \cup \sigma_{1}^{*}(\widetilde{y})$ with $\sigma_{0}^{*}(\widetilde{y}) \subset\left\{\lambda \in \mathbb{C} ;|\lambda|<O\left(\delta^{1 / 4}(h)\right)\right\}$ and $\sigma_{1}^{*}(\widetilde{y}) \subset\left\{\lambda \in \mathbb{C} ; \operatorname{Re}(\lambda)<-c_{3}\right\}$ for $c_{3}>0$. The corresponding eigenspace $\widetilde{E}_{0}^{*}(\widetilde{y})$ to the spectral set $\left.\sigma_{0}^{*} \widetilde{\boldsymbol{y}}\right)$ is spanned by $\left\{\widetilde{\Phi}_{j}^{*}(\widetilde{y})\right\}(j=1, \ldots, 6)$ satisfying $P_{1} \widetilde{\Phi}_{j}^{*}(\widetilde{y})(\boldsymbol{x})=\bar{\Phi}_{j}^{*}\left(z_{1}^{*}\right)+O(\delta(h))$, $P_{2} \widetilde{\Phi}_{j}^{*}(\widetilde{y})(\boldsymbol{x})=O(\delta(h))(j=1,2,3)$ and $\left.P_{1} \widetilde{\Phi}_{j}^{*}(\widetilde{y})(\boldsymbol{x})=O(\delta(h)), P_{2} \widetilde{\Phi}_{j}^{*}(\widetilde{y})(\boldsymbol{x})={\bar{\Phi}_{j}^{*}}_{j}^{*} z_{2}^{*}\right)+O(\delta(h))$ $(j=4,5,6)$.

Remark 3.1. Note that $\widetilde{E}_{1}(\widetilde{y})$ is given by $\widetilde{E}_{1}(\widetilde{y})=\left\{\widetilde{U} \in \widetilde{X} ;\left\langle\widetilde{U}, \widetilde{\Phi}_{j}^{*}(\widetilde{y})\right\rangle_{\widetilde{X}}=0(j=1, \ldots, 6)\right\}$. $\widetilde{X}$.

Now we decompose $\widetilde{U} \in \widetilde{X}$ in the neighborhood of the set $\widetilde{\mathcal{M}}:=\left\{\widetilde{S^{1}}\left(\boldsymbol{p}_{1}, \Theta_{1}\right)+\widetilde{S^{2}}\left(\boldsymbol{p}_{2}, \Theta_{2}\right) ; \widetilde{\boldsymbol{y}}=\left(\boldsymbol{p}_{1}, \Theta_{1}, \boldsymbol{p}_{2}, \Theta_{2}\right) \in \widetilde{Y}\right\} \subset$

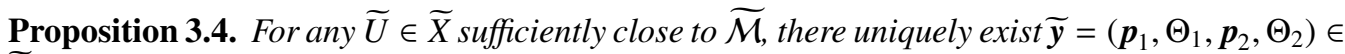
$\widetilde{Y}$ such that

$$
\widetilde{U}=\widetilde{S^{1}}\left(\boldsymbol{p}_{1}, \Theta_{1}\right)+\widetilde{S^{2}}\left(\boldsymbol{p}_{2}, \Theta_{2}\right)+\widetilde{V}
$$

with $\widetilde{V} \in \widetilde{E}_{1}(\widetilde{y})$.

We apply this decomposition to (3.2) and (3.3). Operating $\widetilde{Q}_{0}(\widetilde{y})$ and $\widetilde{Q}_{1}(\widetilde{y})$ on the both sides of (3.3), we have

$$
\begin{gathered}
\widetilde{Q}_{0}(\widetilde{y}) \partial_{t} \widetilde{V}=-\widetilde{Q}_{0}(\widetilde{y}) \dot{\bar{y}}+\widetilde{Q}_{0}(\widetilde{y}) \widetilde{B_{1}} \widetilde{S^{1}}+\widetilde{Q}_{0}(\widetilde{y}) \widetilde{B_{2}} \widetilde{S^{2}}+\widetilde{Q}_{0}(\widetilde{y}) \widetilde{\mathcal{L}}\left(\widetilde{S^{1}}+\widetilde{S^{2}}\right)+\widetilde{Q}_{0}(\widetilde{y}) \widetilde{G_{1}} \\
\widetilde{Q}_{1}(\widetilde{y}) \partial_{t} \widetilde{V}=\widetilde{L}_{S}(\widetilde{y}) \widetilde{V}-\widetilde{Q}_{1}(\widetilde{y}) \dot{\bar{y}}+\widetilde{Q}_{1}(\widetilde{y}) \widetilde{B_{1}} \widetilde{S^{1}}+\widetilde{Q}_{1}(\widetilde{\boldsymbol{y}}) \widetilde{B_{2}} \widetilde{S^{2}}+\widetilde{Q_{1}}(\widetilde{\boldsymbol{y}}) \widetilde{\mathcal{L}}\left(\widetilde{S^{1}}+\widetilde{S^{2}}\right)+\widetilde{Q_{1}}(\widetilde{y}) \widetilde{G_{1}}
\end{gathered}
$$

Proposition 3.5. We have

$$
{ }^{t}\left(\dot{p}_{1}, \dot{\Theta}_{1},-B_{1}\left(\boldsymbol{p}_{1}, \Theta_{1}\right) S^{1}\right)=\beta_{1} \bar{\Phi}_{1}\left(\boldsymbol{p}_{1}, \Theta_{1}\right)+\beta_{2} \bar{\Phi}_{2}\left(\boldsymbol{p}_{1}, \Theta_{1}\right)+\dot{\Theta}_{1} \bar{\Phi}_{3}\left(\boldsymbol{p}_{1}, \Theta_{1}\right)
$$

and

$$
{ }^{t}\left(\dot{p}_{2}, \dot{\Theta}_{2},-B_{2}\left(p_{2}, \Theta_{2}\right) S^{2}\right)=\beta_{1}^{\prime} \bar{\Phi}_{1}\left(p_{2}, \pi-\Theta_{2}\right)+\beta_{2}^{\prime} \bar{\Phi}_{2}\left(p_{2}, \pi-\Theta_{2}\right)+\dot{\Theta}_{2} \bar{\Phi}_{3}\left(p_{2}, \pi-\Theta_{2}\right)
$$

hold, where $\beta_{1}=\left(\dot{p}_{1} \cos \Theta_{1}+\dot{q}_{1} \sin \Theta_{1}\right), \beta_{2}=\left(-\dot{p}_{1} \sin \Theta_{1}+\dot{q}_{1} \cos \Theta_{1}\right)$ and $\beta_{1}^{\prime}=\left(-\dot{p}_{2} \cos \Theta_{2}+\right.$ $\left.\dot{q}_{2} \sin \Theta_{2}\right), \beta_{2}^{\prime}=-\left(\dot{p}_{2} \sin \Theta_{2}+\dot{q}_{2} \cos \Theta_{2}\right)$. 
Proof. The facts $B_{1} S^{1}=\beta_{1} \partial_{z_{1}} S^{1}+\beta_{2} \partial_{z_{2}} S^{1}+\dot{\Theta}_{1} \partial_{R} S^{1}$ and $\beta_{1} R_{1}\left(\Theta_{1}\right) \boldsymbol{e}_{1}+\beta_{2} R_{1}\left(\Theta_{1}\right) \boldsymbol{e}_{2}=\dot{p}_{1}$ lead to the proof. Similarly, The facts $B_{2} S^{2}=\beta_{1}^{\prime} \partial_{z_{1}} S^{2}+\beta_{2}^{\prime} \partial_{z_{2}} S^{2}+\dot{\Theta}_{2} \partial_{R} S^{2}$ and $\beta_{1}^{\prime} R_{2}\left(\Theta_{2}\right) \boldsymbol{e}_{1}+$ $\beta_{2}^{\prime} R_{2}\left(\Theta_{1}\right) \boldsymbol{e}_{2}=\dot{p}_{2}$ lead to the proof.

By Propositions 3.2 and 3.5, $\left.\widetilde{Q}_{0}\left(\dot{\bar{y}}_{1}-\widetilde{B_{1}} \widetilde{S^{1}}\right)=\beta_{1} \widetilde{\Phi}_{1}(\widetilde{y})+\beta_{2} \widetilde{\Phi}_{2} \widetilde{y}\right)+\dot{\Theta}_{1} \widetilde{\Phi}_{3}(\widetilde{y})+O(\delta(h))$ and $\widetilde{Q}_{0}\left(\dot{\bar{y}}_{2}-\widetilde{B_{2}} \widetilde{S^{2}}\right)=\beta_{1}^{\prime} \widetilde{\Phi}_{4}(\widetilde{y})+\beta_{2}^{\prime} \widetilde{\Phi}_{5}(\widetilde{y})+\dot{\Theta}_{2} \widetilde{\Phi}_{6}(\widetilde{y})+O(\delta(h)), \widetilde{Q}_{1}\left(\dot{\bar{y}}_{1}-\widetilde{B_{1}} \widetilde{S^{1}}\right)=O(\delta(h))$ and $\widetilde{Q}_{1}\left(\dot{\bar{y}}_{2}-\widetilde{B_{2} S^{2}}\right)=O(\delta(h))$ hold. The direct calculation also leads to $\widetilde{\mathcal{L}}\left(\widetilde{S^{1}}+\widetilde{S^{2}}\right)=O(\delta(h))$ and therefore for (3.4) and (3.5),

$$
\begin{aligned}
\widetilde{Q}_{0}(\widetilde{y}) \partial_{t} \widetilde{V}= & -\left\{\beta_{1} \widetilde{\Phi}_{1}(\widetilde{y})+\beta_{2} \widetilde{\Phi}_{2}(\widetilde{y})+\dot{\Theta}_{1} \widetilde{\Phi}_{3}(\widetilde{y})\right. \\
& \left.+\beta_{1}^{\prime} \widetilde{\Phi}_{4}(\widetilde{y})+\beta_{2}^{\prime} \widetilde{\Phi}_{5}(\widetilde{y})+\dot{\Theta}_{2} \widetilde{\Phi}_{6}(\widetilde{y})\right\}+\widetilde{Q}_{0}(\widetilde{y}) \widetilde{G_{1}}+O(\delta(h)),
\end{aligned}
$$

and

$$
\left.\widetilde{Q}_{1}(\widetilde{y}) \partial_{t} \widetilde{V}=\widetilde{L}_{S} \widetilde{\boldsymbol{y}}\right) \widetilde{V}+\widetilde{H}_{1}\left(\widetilde{S^{1}}, \widetilde{S^{2}}, \widetilde{V}\right)
$$

hold with $\widetilde{V} \in \widetilde{E}_{1}(\widetilde{y})$ and $\left|\widetilde{H}_{1}\right| \leq O\left(\delta(h)+|\widetilde{V}|^{2}\right)$. Fixing $\widetilde{\boldsymbol{y}}_{0} \in \widetilde{Y}$ arbitrarily, we define the map $\Pi(\widetilde{\boldsymbol{y}}): \widetilde{E}_{1}\left(\widetilde{\boldsymbol{y}}_{0}\right) \rightarrow \widetilde{E}_{1}(\widetilde{\boldsymbol{y}})$ as follows. Let $\widetilde{\boldsymbol{y}}^{*}(\eta):=(1-\eta) \widetilde{\boldsymbol{y}}_{0}+\eta \widetilde{\boldsymbol{y}}$ for $0 \leq \eta \leq 1, \widetilde{a}_{i j}(\eta):=$ $\left.\left.\left\langle\widetilde{\Phi}_{i}^{*} \widetilde{\boldsymbol{y}}^{*}(\eta)\right), \widetilde{\Phi}_{j} \widetilde{\boldsymbol{y}}^{*}(\eta)\right)\right\rangle_{2}$ and $\widetilde{M}(\eta):=\left\{\widetilde{a}_{i j}(\eta)\right\}_{1 \leq i, j \leq 6}$. Note that $\widetilde{\boldsymbol{y}}^{*}(\eta) \in \widetilde{Y}$ for $0 \leq \eta \leq 1$ and $\widetilde{M}(\eta)$ is invertible for $0 \leq \eta \leq 1$ by assumption (H4). We define the map $\Pi(\widetilde{y}) \widetilde{W}_{0}$ for $\widetilde{W}_{0} \in \widetilde{E}_{1}\left(\widetilde{\boldsymbol{y}}_{0}\right.$ ) by $\Pi \widetilde{y}) \widetilde{W}_{0}:=\widetilde{W}(1)$, where $\widetilde{W}(\eta)$ is the solution of

$$
\left\{\begin{aligned}
\frac{d \widetilde{W}}{d \eta} & =\sum_{j=1}^{6} c_{j}(\eta, \widetilde{W}) \widetilde{\Phi}_{j}\left(\widetilde{\boldsymbol{y}}^{*}(\eta)\right), \\
\widetilde{W}(0) & =\widetilde{W}_{0}
\end{aligned}\right.
$$

with $\left.\left.^{t}\left(c_{1}, \ldots, c_{6}\right)(\eta, \widetilde{W}):=-\widetilde{M}^{-1}(\eta)^{t}\left(\left\langle\widetilde{W}, \partial_{\eta} \widetilde{\Phi}_{1}^{*} \widetilde{\boldsymbol{y}}^{*}(\eta)\right)\right\rangle_{2}, \ldots,\left\langle\widetilde{W}, \partial_{\eta} \widetilde{\Phi}_{6}^{*} \widetilde{\boldsymbol{y}}^{*}(\eta)\right)\right\rangle_{2}\right)$. Then $\Pi(\widetilde{\boldsymbol{y}})$ is a homeomorphism from $\widetilde{E}_{1}\left(\widetilde{\boldsymbol{y}}_{0}\right)$ to $\widetilde{E}_{1}(\widetilde{\boldsymbol{y}})$ (see [36]). Transforming $\left.\widetilde{V}(t)=\Pi \widetilde{\boldsymbol{y}}(t)\right) \widetilde{W}(t)$, we see

$$
\partial_{t} \widetilde{V}=\left(\partial_{\widetilde{\boldsymbol{y}}} \Pi(\widetilde{\boldsymbol{y}}(t)) \dot{\boldsymbol{y}}(t)\right) \widetilde{W}+\Pi(\widetilde{\boldsymbol{y}}(t)) \partial_{t} \widetilde{W},
$$

and, hence,

$$
\left.\left.\left.\widetilde{Q}_{0} \widetilde{\boldsymbol{y}}\right) \partial_{t} \widetilde{V}=\widetilde{Q}_{0} \widetilde{\boldsymbol{y}}\right)\left(\partial_{\widetilde{\boldsymbol{y}}} \Pi \widetilde{\boldsymbol{y}}(t)\right) \dot{\boldsymbol{y}}(t)\right) \widetilde{W}=O\left(|\dot{\boldsymbol{y}}(t)| \cdot\|\widetilde{W}\|_{\infty}\right),
$$

$$
\left.\widetilde{Q}_{1}(\widetilde{\boldsymbol{y}}) \partial_{t} \widetilde{V}=\widetilde{Q}_{1}(\widetilde{\boldsymbol{y}})\left(\partial_{\widetilde{\boldsymbol{y}}} \Pi(\widetilde{\boldsymbol{y}}(t)) \dot{\boldsymbol{y}}(t)\right) \widetilde{W}+\widetilde{Q}_{1}(\widetilde{\boldsymbol{y}}) \Pi \widetilde{\boldsymbol{y}}(t)\right) \partial_{t} \widetilde{W}=\Pi(\widetilde{\boldsymbol{y}}(t)) \partial_{t} \widetilde{W}+O\left(|\dot{\boldsymbol{y}}(t)| \cdot\|\widetilde{W}\|_{\infty}\right)
$$

hold. Therefore (3.6) and (3.7) become

$$
\left\{\begin{array}{c}
\beta_{1} \widetilde{\Phi}_{1}(\widetilde{\boldsymbol{y}})+\beta_{2} \widetilde{\Phi}_{2}(\widetilde{\boldsymbol{y}})+\dot{\Theta}_{1} \widetilde{\Phi}_{3}(\widetilde{\boldsymbol{y}})+\beta_{1}^{\prime} \widetilde{\Phi}_{4}(\widetilde{\boldsymbol{y}})+\beta_{2}^{\prime} \widetilde{\Phi}_{5}(\widetilde{\boldsymbol{y}})+\dot{\Theta}_{2} \widetilde{\Phi}_{6}(\widetilde{\boldsymbol{y}}) \\
\quad=O\left(\delta(h)+\|\widetilde{W}\|_{\infty}^{2}+|\dot{\overline{\boldsymbol{y}}}(t)| \cdot\|\widetilde{W}\|_{\infty}\right), \\
\partial_{t} \widetilde{W}=\widetilde{A}(\widetilde{y}) \widetilde{W}+O\left(\delta(h)+\|\widetilde{W}\|_{\infty}^{2}+|\dot{\bar{y}}(t)| \cdot\|\widetilde{W}\|_{\infty}\right),
\end{array}\right.
$$

where $\widetilde{A}(\widetilde{y}):=\Pi^{-1}(\widetilde{y}) \widetilde{L}_{S}(\widetilde{y}) \Pi(\widetilde{y})$. Let $\widetilde{X}^{\omega}$ for $0<\omega<1$ be the fractional powered space with respect to $\widetilde{A}(\widetilde{y})$ embedded into $L^{\infty}\left(\mathbb{R}^{2}\right)$ and $\widetilde{X}\left(D_{1}\right):=\left\{\widetilde{W} \in \widetilde{E}_{1}\left(\widetilde{y}_{0}\right) ;\|\widetilde{W}\|_{\omega} \leq D_{1} \delta(h)\right\}$.

Propositions 3.1 and 3.2 and [36] give the following result. 
Proposition 3.6. There exists $D_{1}>0$ such that the solution $(\widetilde{\boldsymbol{y}}(t), \widetilde{W}(t))$ of (3.8) satisfies $\dot{\bar{y}}=$ $O(\delta(h(t)))$ and $\|\widetilde{W}(t)\|_{\omega} \leq D_{1} \delta(h(t))$ uniformly for $t \geq 0$ as long as $\widetilde{\boldsymbol{y}}={ }^{t}\left(\boldsymbol{p}_{1}, \Theta_{1}, \boldsymbol{p}_{2}, \Theta_{2}\right) \in \widetilde{Y}$.

Let $\overline{\mathcal{L}}_{1}(\bar{U})$ be the right-hand side of (2.4) replaced $R(\Theta)$ by $R_{1}(\Theta)$ and $\overline{\mathcal{L}}_{2}(\bar{U})$ be the one replaced by $R_{2}(\Theta)$ for $\bar{U}={ }^{t}(\boldsymbol{p}, \Theta, u)$. Then we have the following theorem.

Theorem 3.1. If the initial data $\widetilde{U}_{0}$ is sufficiently close to $\widetilde{S^{1}}\left(\boldsymbol{p}_{1}^{0}, \Theta_{1}^{0}\right)+\widetilde{S^{2}}\left(\boldsymbol{p}_{2}^{0}, \Theta_{2}^{0}\right)$ for ${ }^{t}\left(\boldsymbol{p}_{1}^{0}, \Theta_{1}^{0}, \boldsymbol{p}_{2}^{0}, \Theta_{2}^{0}\right) \in$ $\widetilde{Y}$, then the solution $\widetilde{U}(t, \boldsymbol{x})$ of $(3.1) \partial_{t} \widetilde{U}=\widetilde{\mathcal{L}}(\widetilde{U})$ satisfies

$$
\left\|\left\{\widetilde{U}(t)-\left\{\widetilde{S^{1}}\left(\boldsymbol{p}_{1}(t), \Theta_{1}(t)\right)+\widetilde{S^{2}}\left(\boldsymbol{p}_{2}(t), \Theta_{2}(t)\right)\right\}\right\}\right\|_{\infty} \leq O(\delta(h(t)))
$$

as long as ${ }^{t}\left(\boldsymbol{p}_{1}(t), \Theta_{1}(t), \boldsymbol{p}_{2}(t), \Theta_{2}(t)\right) \in \widetilde{Y}$, where $h(t)=\left|\boldsymbol{p}_{2}(t)-\boldsymbol{p}_{1}(t)\right| .{ }^{t}\left(\boldsymbol{p}_{1}(t), \Theta_{1}(t), \boldsymbol{p}_{2}(t), \Theta_{2}(t)\right)$ satisfies

$$
\bar{M}_{j}\left(\Theta_{j}\right)\left(\begin{array}{c}
R_{j}\left(-\Theta_{j}\right) \dot{\boldsymbol{p}}_{j} \\
\dot{\Theta}_{j}
\end{array}\right)=\overline{\boldsymbol{b}}_{j}(\widetilde{\boldsymbol{y}})+O\left(\delta^{2}(h(t))\right) \quad(j=1,2),
$$

where $\bar{M}_{1}\left(\Theta_{1}\right)=\left\{m_{i j}^{1}\left(\Theta_{1}\right)\right\}$ and $\bar{M}_{2}\left(\Theta_{2}\right)=\left\{m_{i j}^{2}\left(\Theta_{2}\right)\right\}$ are the third-order square matrices with $m_{i j}^{1}\left(\Theta_{1}\right):=\left\langle\bar{\Phi}_{i}^{*}\left(\boldsymbol{p}_{1}, \Theta_{1}\right), \bar{\Phi}_{j}\left(\boldsymbol{p}_{1}, \Theta_{1}\right)\right\rangle_{2}, m_{i j}^{2}\left(\Theta_{2}\right):=\left\langle\bar{\Phi}_{i}^{*}\left(\boldsymbol{p}_{2}, \Theta_{2}\right), \bar{\Phi}_{j}\left(\boldsymbol{p}_{2}, \Theta_{2}\right)\right\rangle_{2}$ independent of $\boldsymbol{p}_{j}$ $(j=1,2)$ and also $\overline{\boldsymbol{b}}_{j}(\widetilde{\boldsymbol{y}})={ }^{t}\left(b_{1}^{j}(\widetilde{\boldsymbol{y}}), b_{2}^{j}(\widetilde{\boldsymbol{y}}), b_{3}^{j}(\widetilde{\boldsymbol{y}})\right) \in \mathbb{R}^{3}$ with

$$
\left\{\begin{array}{rll}
\left.b_{i}^{1} \widetilde{\boldsymbol{y}}\right) & :=\left\langle\left\langle\boldsymbol{K}_{0}, \boldsymbol{e}_{i}\right\rangle \gamma_{1}^{\prime}\left(S^{1}\right), S^{2}\right\rangle_{2}+\left\langle g_{4}\left(S^{1}, S^{2}\right), \Phi_{i}^{*}\left(z_{1}^{*}\right)\right\rangle_{2} & (i=1,2), \\
\left.b_{3}^{1} \widetilde{\boldsymbol{y}}\right) & :=\left\langle k_{0} \gamma_{2}^{\prime}\left(S^{1}\right), S^{2}\right\rangle_{2}+\left\langle g_{4}\left(S^{1}, S^{2}\right), \Phi_{3}^{*}\left(z_{1}^{*}\right)\right\rangle_{2}, & \\
b_{i}^{2}(\widetilde{\boldsymbol{y}}) & :=\left\langle\left\langle\boldsymbol{K}_{0}, \boldsymbol{e}_{i}\right\rangle \gamma_{1}^{\prime}\left(S^{2}\right), S^{1}\right\rangle_{2}+\left\langle g_{4}\left(S^{1}, S^{2}\right), \Phi_{i}^{*}\left(z_{2}^{*}\right)\right\rangle_{2} \quad(i=1,2), \\
\left.b_{3}^{2} \widetilde{\boldsymbol{y}}\right) & :=\left\langle k_{0} \gamma_{2}^{\prime}\left(S^{2}\right), S^{1}\right\rangle_{2}+\left\langle g_{4}\left(S^{1}, S^{2}\right), \Phi_{3}^{*}\left(z_{2}^{*}\right)\right\rangle_{2} &
\end{array}\right.
$$

where $g_{4}\left(S^{1}, S^{2}\right):=f_{0}\left(S^{1}+S^{2}\right)-f_{0}\left(S^{1}\right)-f_{0}\left(S^{2}\right)$.

Proof. The first half of the statement is clear by Proposition 3.6. We will show the latter half on the motion of ${ }^{t}\left(\boldsymbol{p}_{1}(t), \Theta_{1}(t), \boldsymbol{p}_{2}(t), \Theta_{2}(t)\right)$. From equations (3.6), (3.7), (3.8), and Proposition 3.6, we have

$$
\begin{aligned}
\beta_{1} \widetilde{\Phi}_{1}(\widetilde{y})+\beta_{2} \widetilde{\Phi}_{2}(\widetilde{y})+\dot{\Theta}_{1} \widetilde{\Phi}_{3}(\widetilde{y}) & +\beta_{1}^{\prime} \widetilde{\Phi}_{4}(\widetilde{y})+\beta_{2}^{\prime} \widetilde{\Phi}_{5}(\widetilde{y})+\dot{\Theta}_{2} \widetilde{\Phi}_{6}(\widetilde{y}) \\
& \left.=\widetilde{Q}_{0} \widetilde{\boldsymbol{y}}\right) \widetilde{\mathcal{L}}\left(\widetilde{S^{1}}+\widetilde{S^{2}}\right)+O\left(\delta^{2}(h)\right),
\end{aligned}
$$

which is equivalent to

$$
\begin{array}{r}
\left\langle\beta_{1} \widetilde{\Phi}_{1}(\widetilde{y})+\beta_{2} \widetilde{\Phi}_{2}(\widetilde{y})+\dot{\Theta}_{1} \widetilde{\Phi}_{3}(\widetilde{y})+\beta_{1}^{\prime} \widetilde{\Phi}_{4}(\widetilde{y})+\beta_{2}^{\prime} \widetilde{\Phi}_{5}(\widetilde{y})+\dot{\Theta}_{2} \widetilde{\Phi}_{6}(\widetilde{y}), \widetilde{\Phi}_{i}^{*}(\widetilde{y})\right\rangle_{2} \\
=\left\langle\widetilde{\mathcal{L}}\left(\widetilde{S^{1}}+\widetilde{S^{2}}\right), \widetilde{\Phi}_{i}^{*}(\widetilde{y})\right\rangle_{2}+O\left(\delta^{2}(h)\right) \quad(i=1, \ldots, 6),
\end{array}
$$

and is written as

$$
\widetilde{M}(\widetilde{y}) \widetilde{\beta}=\widetilde{\boldsymbol{b}}(\widetilde{y}),
$$

where $\left.\widetilde{M}(\widetilde{\boldsymbol{y}}):=\left\{\widetilde{m}_{i j}(\widetilde{\boldsymbol{y}})\right\}_{1 \leq i, j \leq 6}, \widetilde{m}_{i j}(\widetilde{\boldsymbol{y}}):=\left\langle\widetilde{\Phi}_{i}^{*}(\widetilde{\boldsymbol{y}}), \widetilde{\Phi}_{j}(\widetilde{\boldsymbol{y}})\right\rangle_{2}, \widetilde{\boldsymbol{\beta}}:={ }^{t}\left(\beta_{1}, \beta_{2}, \dot{\Theta}_{1}, \beta_{1}^{\prime}, \beta_{2}^{\prime}, \dot{\Theta}_{2}\right), \widetilde{\boldsymbol{b}} \widetilde{\boldsymbol{y}}\right)=$ $\widetilde{\boldsymbol{b}}_{0}(\widetilde{\boldsymbol{y}})+O\left(\delta^{2}(h)\right), \widetilde{\boldsymbol{b}}_{0}(\widetilde{\boldsymbol{y}})={ }^{t}\left(\widetilde{b}_{1}^{0}(\widetilde{\boldsymbol{y}}), \cdots, \widetilde{b}_{6}^{0}(\widetilde{\boldsymbol{y}})\right)$ with $\widetilde{b}_{i}^{0}(\widetilde{\boldsymbol{y}}):=\left\langle\widetilde{\mathcal{L}}\left(\widetilde{S^{1}}+\widetilde{S^{2}}\right), \widetilde{\Phi}_{i}^{*}(\widetilde{\boldsymbol{y}})\right\rangle_{2}$. 
By Propositions 3.2 and 3.3, it follows that

$$
\widetilde{m}_{i j}(\boldsymbol{y})=\left\{\begin{array}{l}
\left\langle\bar{\Phi}_{i}^{*}\left(p_{1}, \Theta_{1}\right), \bar{\Phi}_{j}\left(p_{1}, \Theta_{1}\right)\right\rangle_{2}+O(\delta(h)) \quad(1 \leq i, j \leq 3), \\
O(\delta(h)) \quad(1 \leq i \leq 3,4 \leq j \leq 6),(4 \leq i \leq 6,1 \leq j \leq 3), \\
\left\langle\bar{\Phi}_{i-3}^{*}\left(p_{2}, \pi-\Theta_{2}\right), \bar{\Phi}_{j-3}\left(p_{2}, \pi-\Theta_{2}\right)\right\rangle_{2}+O(\delta(h)) \quad(4 \leq i, j \leq 6) .
\end{array}\right.
$$

Note that Proposition 2.2 and Remark 2.3 give

$$
\left\langle\bar{\Phi}_{i}^{*}\left(\boldsymbol{p}_{1}, \Theta_{1}\right), \bar{\Phi}_{j}\left(\boldsymbol{p}_{1}, \Theta_{1}\right)\right\rangle_{2}=\left\langle\boldsymbol{e}_{i}, R_{1}\left(\Theta_{1}\right) \boldsymbol{e}_{j}\right\rangle-\left\langle\Phi_{i}^{*}, \partial_{z_{j}} S^{*}\right\rangle_{2}=: m_{i j}^{0}\left(\Theta_{1}\right)
$$

for $i, j=1,2,\left\langle\bar{\Phi}_{3}^{*}\left(\boldsymbol{p}_{1}, \Theta_{1}\right), \bar{\Phi}_{j}\left(\boldsymbol{p}_{1}, \Theta_{1}\right)\right\rangle_{2}=-\left\langle\Phi_{3}^{*}, \partial_{z_{j}} S^{*}\right\rangle_{2}=: m_{3 j}^{0}$ and $\left\langle\bar{\Phi}_{i}^{*}\left(\boldsymbol{p}_{1}, \Theta_{1}\right), \bar{\Phi}_{3}\left(\boldsymbol{p}_{1}, \Theta_{1}\right)\right\rangle_{2}=$ $-\left\langle\Phi_{i}^{*}, \partial_{R} S^{*}\right\rangle_{2}=: m_{i 3}^{0}$ for $i, j=1,2$, and $\left\langle\bar{\Phi}_{3}^{*}\left(\boldsymbol{p}_{1}, \Theta_{1}\right), \bar{\Phi}_{3}\left(\boldsymbol{p}_{1}, \Theta_{1}\right)\right\rangle_{2}=1-\left\langle\Phi_{3}^{*}, \partial_{R} S^{*}\right\rangle_{2}=: m_{33}^{0}$. Similarly, we have

$$
\left\langle\bar{\Phi}_{i-3}^{*}\left(\boldsymbol{p}_{2}, \pi-\Theta_{2}\right), \bar{\Phi}_{j-3}\left(\boldsymbol{p}_{2}, \pi-\Theta_{2}\right)\right\rangle_{2}=\left\langle\boldsymbol{e}_{i-3}, R_{2}\left(\Theta_{2}\right) \boldsymbol{e}_{j-3}\right\rangle-\left\langle\Phi_{i-3}^{*}, \partial_{z_{j-3}} S^{*}\right\rangle_{2}=: m_{i j}^{0}\left(\Theta_{2}\right)
$$

for $i, j=4,5, m_{6 j}^{0}:=\left\langle\bar{\Phi}_{3}^{*}\left(\boldsymbol{p}_{2}, \pi-\Theta_{2}\right), \bar{\Phi}_{j-3}\left(\boldsymbol{p}_{2}, \pi-\Theta_{2}\right)\right\rangle_{2}=-\left\langle\Phi_{3}^{*}, \partial_{z_{j-3}} S^{*}\right\rangle_{2}=m_{3(j-3)}^{0}$ and $m_{i 6}^{0}:=\left\langle\bar{\Phi}_{i-3}^{*}\left(\boldsymbol{p}_{2}, \pi-\Theta_{2}\right), \bar{\Phi}_{3}\left(\boldsymbol{p}_{2}, \pi-\Theta_{2}\right)\right\rangle_{2}=-\left\langle\Phi_{i-3}^{*}, \partial_{R} S^{*}\right\rangle_{2}=m_{(i-3) 3}^{0}$ for $i, j=4,5$, and $m_{66}^{0}:=\left\langle\Phi_{3}^{*}\left(\boldsymbol{p}_{2}, \pi-\Theta_{2}\right), \bar{\Phi}_{3}\left(\boldsymbol{p}_{2}, \pi-\Theta_{2}\right)\right\rangle_{2}=1-\left\langle\Phi_{3}^{*}, \partial_{R} S^{*}\right\rangle_{2}=m_{33}^{0}$. Thus, $\widetilde{M}(\widetilde{y})=\widetilde{M}_{0}(\widetilde{y})+$ $O(\delta(h))$ and $\left.\widetilde{M_{0}} \widetilde{y}\right)=\left(\begin{array}{cc}\overline{M_{1}}\left(\Theta_{1}\right) & O \\ O & \overline{M_{2}}\left(\Theta_{2}\right)\end{array}\right)$, where $\overline{M_{1}}\left(\Theta_{1}\right)=\left\{m_{i j}^{0}\right\}_{1 \leq i, j \leq 3}, \overline{M_{2}}\left(\Theta_{2}\right)=\left\{m_{i j}^{0}\right\}_{4 \leq i, j \leq 6}$, and $O$ denotes the zero matrix. Then from (3.11) we have

$$
\widetilde{M}_{0}(\widetilde{\boldsymbol{y}}) \widetilde{\boldsymbol{\beta}}=\widetilde{\boldsymbol{b}}_{0}+O\left(\delta^{2}(h)\right) .
$$

Since $^{t}\left(\beta_{1}, \beta_{2}\right)=R_{1}\left(-\Theta_{1}\right) \dot{p}_{1}$ and ${ }^{t}\left(\beta_{1}^{\prime}, \beta_{2}^{\prime}\right)=R_{2}\left(-\Theta_{2}\right) \dot{p}_{2}$ hold, we have

$$
\widetilde{M}_{0}(\widetilde{y}) \widetilde{\boldsymbol{\beta}}={ }^{t}\left(\overline{M_{1}}\left(\Theta_{1}\right)\left(\begin{array}{c}
R_{1}\left(-\Theta_{1}\right) \dot{\boldsymbol{p}}_{1} \\
\dot{\Theta}_{1}
\end{array}\right), \overline{M_{2}}\left(\Theta_{2}\right)\left(\begin{array}{c}
R_{2}\left(-\Theta_{2}\right) \dot{\boldsymbol{p}}_{2} \\
\dot{\Theta}_{2}
\end{array}\right)\right)
$$

Next, we consider $\widetilde{\boldsymbol{b}}_{0}$. Now, $P_{1} \widetilde{\mathcal{L}}\left(\widetilde{S^{1}}+\widetilde{S^{2}}\right)=\overline{\mathcal{L}}_{1}\left(\overline{S_{1}}+{ }^{t}\left(\mathbf{0}, 0, S^{2}\right)\right)+{ }^{t}\left(\mathbf{0}, 0, f_{1}\left(z_{2}^{*}\right)\right)$ and $P_{2} \widetilde{\mathcal{L}}\left(\widetilde{S^{1}}+\widetilde{S^{2}}\right)=\overline{\mathcal{L}}_{2}\left({ }^{t}\left(\mathbf{0}, 0, S_{1}\right)+\overline{S^{2}}\right)+{ }^{t}\left(\mathbf{0}, 0, f_{1}\left(z_{1}^{*}\right)\right)$ hold. Then combining with Proposition 3.3 , we see

$$
\widetilde{b}_{i}^{0}(\tilde{y})=\left\{\begin{array}{cl}
\left\langle\overline{\mathcal{L}}_{1}\left(\overline{S_{1}}+{ }^{t}\left(\mathbf{0}, 0, S^{2}\right)\right), \bar{\Phi}_{i}^{*}\left(z_{1}^{*}\right)\right\rangle_{2}+\left\langle f_{1}\left(z_{2}^{*}\right), \Phi_{i}^{*}\left(z_{1}^{*}\right)\right\rangle_{2}+O\left(\delta^{2}(h)\right) & (1 \leq i \leq 3), \\
\left\langle\overline{\mathcal{L}}_{2}\left(t\left(\mathbf{0}, 0, S^{1}\right)+\bar{S}_{2}\right), \bar{\Phi}_{i-3}^{*}\left(z_{2}^{*}\right)\right\rangle_{2}+\left\langle f_{1}\left(z_{1}^{*}\right), \Phi_{i-3}^{*}\left(z_{2}^{*}\right)\right\rangle_{2}+O\left(\delta^{2}(h)\right) & (4 \leq i \leq 6) .
\end{array}\right.
$$

Since $\gamma_{j}\left(S^{1}+S^{2}\right)=\gamma_{j}\left(S^{1}\right)+\gamma_{j}^{\prime}\left(S^{1}\right) S^{2}+O\left(\delta^{2}(h)\right)$ holds on $\Omega_{1}$, equation (2.15) gives

$$
\begin{aligned}
& \left\langle\overline{\mathcal{L}}_{1}\left(\overline{S_{1}}+{ }^{t}\left(\mathbf{0}, 0, S^{2}\right)\right), \bar{\Phi}_{i}^{*}\left(z_{1}^{*}\right)\right\rangle_{2}+\left\langle f_{1}\left(z_{2}^{*}\right), \Phi_{i}^{*}\left(z_{1}^{*}\right)\right\rangle_{2} \\
& =\left\langle\left\langle\boldsymbol{K}_{0}, \boldsymbol{e}_{i}\right\rangle \gamma_{1}^{\prime}\left(S^{1}\right), S^{2}\right\rangle_{2} \\
& \quad+\left\langle d \Delta\left(S^{1}+S^{2}\right)+f_{0}\left(S^{1}+S^{2}\right)+f_{1}\left(z_{1}^{*}\right)+f_{1}\left(z_{2}^{*}\right), \Phi_{i}^{*}\left(z_{1}^{*}\right)\right\rangle_{2}+O\left(\delta^{2}(h)\right) \\
& =\quad\left\langle\left\langle\boldsymbol{K}_{0}, \boldsymbol{e}_{i}\right\rangle \gamma_{1}^{\prime}\left(S^{1}\right), S^{2}\right\rangle_{2}+\left\langle f_{0}\left(S^{1}+S^{2}\right)-f_{0}\left(S^{1}\right)-f_{0}\left(S^{2}\right), \Phi_{i}^{*}\left(z_{1}^{*}\right)\right\rangle_{2}+O\left(\delta^{2}(h)\right)
\end{aligned}
$$


for $i=1,2$ and

$$
\begin{aligned}
& \left\langle\overline{\mathcal{L}}_{1}\left(\overline{S_{1}}+{ }^{t}\left(\mathbf{0}, 0, S^{2}\right)\right), \bar{\Phi}_{3}^{*}\left(z_{1}^{*}\right)\right\rangle_{2}+\left\langle f_{1}\left(z_{2}^{*}\right), \Phi_{3}^{*}\left(z_{1}^{*}\right)\right\rangle_{2} \\
& \quad=\left\langle k_{0} \gamma_{2}^{\prime}\left(S^{1}\right), S^{2}\right\rangle_{2}+\left\langle f_{0}\left(S^{1}+S^{2}\right)-f_{0}\left(S^{1}\right)-f_{0}\left(S^{2}\right), \Phi_{3}^{*}\left(z_{1}^{*}\right)\right\rangle_{2}+O\left(\delta^{2}(h)\right) .
\end{aligned}
$$

Similarly,

$$
\begin{aligned}
& \left\langle\overline{\mathcal{L}}_{2}\left({ }^{t}\left(\mathbf{0}, 0, S^{1}\right)+\overline{S_{2}}\right), \bar{\Phi}_{i-3}^{*}\left(z_{2}^{*}\right)\right\rangle_{2}+\left\langle f_{1}\left(z_{1}^{*}\right), \Phi_{i-3}^{*}\left(z_{2}^{*}\right)\right\rangle_{2} \\
& \quad=\left\langle\left\langle\boldsymbol{K}_{0}, \boldsymbol{e}_{i-3}\right\rangle \gamma_{1}^{\prime}\left(S^{2}\right), S^{1}\right\rangle_{2}+\left\langle f_{0}\left(S^{1}+S^{2}\right)-f_{0}\left(S^{1}\right)-f_{0}\left(S^{2}\right), \Phi_{i-3}^{*}\left(z_{2}^{*}\right)\right\rangle_{2}+O\left(\delta^{2}(h)\right)
\end{aligned}
$$

for $i=4,5$ and

$$
\begin{aligned}
& \left\langle\overline{\mathcal{L}}_{2}\left({ }^{t}\left(\mathbf{0}, 0, S^{1}\right)+\overline{S_{2}}\right), \bar{\Phi}_{3}^{*}\left(z_{2}^{*}\right)\right\rangle_{2}+\left\langle f_{1}\left(z_{1}^{*}\right), \Phi_{3}^{*}\left(z_{2}^{*}\right)\right\rangle_{2} \\
& \quad=\left\langle k_{0} \gamma_{2}^{\prime}\left(S^{2}\right), S^{1}\right\rangle_{2}+\left\langle f_{0}\left(S^{1}+S^{2}\right)-f_{0}\left(S^{1}\right)-f_{0}\left(S^{2}\right), \Phi_{3}^{*}\left(z_{2}^{*}\right)\right\rangle_{2}+O\left(\delta^{2}(h)\right)
\end{aligned}
$$

hold.

Thus, the proof is complete.

To derive an explicit motion of interaction, we assume the shape of the camphor particle is almost radially symmetric. Let $\boldsymbol{e}(\theta):=\left(\begin{array}{c}\cos \theta \\ \sin \theta\end{array}\right)$ and assume

$$
\partial \Omega_{0}=\left\{\left(r_{0}+\varepsilon b(\theta)\right) \boldsymbol{e}(\theta)\right\}
$$

for $r_{0}>0,0<\varepsilon \ll 1$, and $b(\theta):=\cos m_{0} \theta$ with $2 \leq m_{0} \in \mathbb{N}$. Then, the stationary solution $S^{*}(\boldsymbol{x})=S_{\varepsilon}^{*}(\boldsymbol{x})$ is given by

$$
S_{\varepsilon}^{*}(r e(\theta))=U_{0}(r)+\varepsilon b(\theta) U_{1}(r)+\varepsilon^{2} U_{2}(r, \theta)
$$

from (2.19) and (2.20). Here $U_{0}(r), U_{1}(r)$, and $U_{2}(r, \theta)$ have the asymptotic profiles as $r \rightarrow \infty$ and the estimate

$$
U_{j}(r) \rightarrow \frac{c_{j}^{\prime}}{\sqrt{r}} e^{-\alpha_{0} r} \quad(j=0,1), \quad\left|U_{2}(r, \theta)\right| \leq \frac{c_{2}^{\prime}}{\sqrt{1+r}} e^{-\alpha_{0} r}
$$

for positive constants $c_{j}^{\prime}$ and $\alpha_{0}$. In fact, (3.15) generally holds by the assumption $f_{0}^{\prime}(u)<0$, which is shown by using the properties of modified Bessel function of second kind. In addition, $\Phi_{j}^{*}$ have similar expressions as $\Phi_{1}^{*}(r \boldsymbol{e}(\theta))=U_{0}^{*}(r) \cos \theta+\varepsilon U_{1}^{*}(r, \theta), \Phi_{2}^{*}(r \boldsymbol{e}(\theta))=U_{0}^{*}(r) \sin \theta+$ $\varepsilon U_{2}^{*}(r, \theta)$, and $\Phi_{3}^{*}(r e(\theta))=\varepsilon V_{1}^{*}(r) \sin m_{0} \theta+\varepsilon^{2} V_{2}^{*}(r, \theta)$ with

$$
U_{0}^{*}(r) \rightarrow \frac{c_{0}^{*}}{\sqrt{r}} e^{-\alpha_{0} r}, \quad V_{1}^{*}(r) \rightarrow \frac{c_{1}^{*}}{\sqrt{r}} e^{-\alpha_{0} r}, \quad\left|U_{1}^{*}(r, \theta)\right|,\left|V_{2}^{*}(r, \theta)\right| \leq \frac{c_{2}^{*}}{\sqrt{1+r}} e^{-\alpha_{0} r}
$$

as $r \rightarrow \infty$ for positive constants $c_{j}^{*}$. Hence, we add these properties to assumptions:

(H5) The solutions $S^{*}(\boldsymbol{x})$ of (2.19), (2.20), and $\Phi_{j}^{*}(\boldsymbol{x})$ in Proposition 2.6 have the asymptotic profiles (3.15) and (3.16).

Define the directional angle of the vector $\boldsymbol{p}_{2}-\boldsymbol{p}_{1}$ with the horizontal axis by $\Theta_{H}$ as in Fig. 3 and set $\Xi_{j}:=\Theta_{j} \mp \Theta_{H}$ for $j=1,2$. Then we have the following result. 


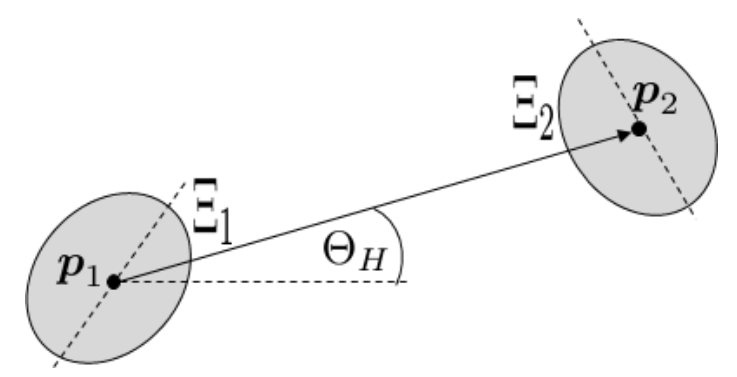

Figure 4: Two camphor particles.

Theorem 3.2. Under assumptions (H3) and (H5) for radially symmetric solutions of (2.19), $\dot{\Xi}_{j}=\frac{\varepsilon}{\sqrt{h}} e^{-\alpha_{0} h} N_{m_{0}} \sin m_{0} \Xi_{j}\left(1+O\left(\frac{1}{h}+\varepsilon+e^{-\alpha_{1} h}\right)\right)$ holds for a positive constant $\alpha_{1}$ as long as $h=\left|\boldsymbol{p}_{2}-\boldsymbol{p}_{1}\right|$ is sufficiently large, where $N_{m}$ is the constant defined by $N_{m_{0}}:=N_{m_{0}}^{\prime}+N_{m_{0}}^{\prime \prime}$ and

$$
\begin{aligned}
& N_{m_{0}}^{\prime}:=-m_{0} c_{0}^{\prime} \int_{0}^{\infty} \int_{0}^{2 \pi} r \xi\left(r-r_{0}\right) \gamma_{2}^{\prime}\left(U_{0}(r)\right) \cos m_{0} \theta e^{\alpha_{0} r \cos \theta} d \theta d r \\
& N_{m_{0}}^{\prime \prime}:=-\int_{0}^{\infty} \int_{0}^{2 \pi} r\left\{f_{0}^{\prime}\left(U_{0}(r)\right)-f_{0}^{\prime}(0)\right\} V_{1}^{*}(r) \cos m_{0} \theta e^{\alpha_{0} r \cos \theta} d \theta d r .
\end{aligned}
$$

Proof. We apply Theorem 3.1 to this case together with results of Propositions 2.2 - 2.6. First, we can easily find

$$
\left\langle\bar{\Phi}_{i}^{*}(\boldsymbol{p}, \Theta), \bar{\Phi}_{j}(\boldsymbol{p}, \Theta)\right\rangle_{2}=\left\{\begin{array}{c}
\cos \Theta-\frac{1}{2} c_{4}+O(\varepsilon)(i=j=1,2), \\
1+O\left(\varepsilon^{2}\right) \quad(i=j=3) \\
-\sin \Theta+O(\varepsilon) \quad(i=1, j=2), \\
\sin \Theta+O(\varepsilon) \quad(i=2, j=1), \\
O(\varepsilon)(i \neq j=3,3=i \neq j),
\end{array}\right.
$$

where $c_{4}:=\left\langle U_{0}^{\prime}, U_{0}^{*}\right\rangle_{2}=2 \pi \int_{0}^{\infty} r U_{0}^{\prime}(r) U_{0}^{*}(r) d r$. Thus, we have

$$
\bar{M}_{1}\left(\Theta_{1}\right)=\left(\begin{array}{ccc}
\cos \Theta_{1}-\frac{1}{2} c_{4} & -\sin \Theta_{1} & 0 \\
\sin \Theta_{1} & \cos \Theta_{1}-\frac{1}{2} c_{4} & 0 \\
0 & 0 & 1
\end{array}\right)+O(\varepsilon),
$$

and

$$
\bar{M}_{2}\left(\Theta_{2}\right)=\left(\begin{array}{ccc}
-\cos \Theta_{2}-\frac{1}{2} c_{4} & -\sin \Theta_{2} & 0 \\
\sin \Theta_{2} & -\cos \Theta_{2}-\frac{1}{2} c_{4} & 0 \\
0 & 0 & 1
\end{array}\right)+O(\varepsilon)
$$

Next, we consider $\overline{\boldsymbol{b}}_{j}(\widetilde{\boldsymbol{y}})$ in Theorem 3.1 and first $b_{3}^{1}(\widetilde{\boldsymbol{y}})$ in (3.9). Putting $\boldsymbol{H}=h \boldsymbol{e}\left(\Theta_{H}\right):=\boldsymbol{p}_{2}-\boldsymbol{p}_{1}$ and $\Xi:=\pi-\left(\Theta_{1}+\Theta_{2}\right)$, we see for $z_{1}^{*}=r \boldsymbol{e}(\theta)$

$$
\begin{aligned}
z_{2}^{*} & =R_{2}\left(-\Theta_{2}\right)\left(\boldsymbol{x}-\boldsymbol{p}_{2}\right) \\
& =R_{1}(-\Xi) R_{1}\left(-\Theta_{1}\right)\left(\boldsymbol{x}-\boldsymbol{p}_{1}-\boldsymbol{H}\right) \\
& =R_{1}(-\Xi)\left(z_{1}^{*}-R_{1}\left(-\Theta_{1}\right) \boldsymbol{H}\right) \\
& =h\left(\frac{r}{h} \boldsymbol{e}(\theta-\Xi)-\boldsymbol{e}\left(-\Xi-\Theta_{1}+\Theta_{H}\right)\right),
\end{aligned}
$$




$$
\left|z_{2}^{*}\right|^{2}=h^{2}\left(\frac{r^{2}}{h^{2}}-\frac{2 r}{h} \cos \left(\theta+\Theta_{1}-\Theta_{H}\right)+1\right)
$$

and, therefore,

$$
\left|z_{2}^{*}\right|=h\left(1-\frac{r}{h} \cos \left(\theta+\Theta_{1}-\Theta_{H}\right)+O\left(\frac{r^{2}}{h^{2}}\right)\right)
$$

as $h \rightarrow \infty$. Hence, we can obtain

$$
z_{2}^{*}=\left|z_{2}^{*}\right| \boldsymbol{e}(\beta(\theta)),
$$

where $\beta(\theta):=\pi-\Xi-\Theta_{1}+\Theta_{H}+\frac{r}{h} \beta_{1}(\theta)+O\left(\frac{r^{2}}{h^{2}}\right)$ with $\beta_{1}(\theta):=\sqrt{1-\cos ^{2}\left(\pi-\Theta_{1}+\Theta_{H}-\theta\right)}$.

Here, we compute $\left.b_{3}^{1} \widetilde{\boldsymbol{y}}\right)=\left\langle k_{0} \gamma_{2}^{\prime}\left(S^{1}\right), S^{2}\right\rangle_{2}+\left\langle g_{4}\left(S^{1}, S^{2}\right) \text {, } \Phi_{3}^{*}\left(z_{1}^{*}\right)\right\rangle_{2}$. Since

$$
\begin{aligned}
g_{4}\left(S^{1}, S^{2}\right) & =\left\{f_{0}^{\prime}\left(S^{1}\right)-f_{0}^{\prime}(0)\right\} S^{2}+\int_{0}^{1} \int_{0}^{1}\left\{f_{0}^{\prime \prime}\left(S^{1}+\eta_{1} \eta_{2} S^{2}\right)-f_{0}^{\prime \prime}\left(\eta_{1} \eta_{2} S^{2}\right)\right\} d \eta_{1} d \eta_{2}\left(S^{2}\right)^{2} \\
& =\left\{f_{0}^{\prime}\left(S^{1}\right)-f_{0}^{\prime}(0)\right\} S^{2}+g_{5}\left(S^{1}, S^{2}\right) S^{1}\left(S^{2}\right)^{2}
\end{aligned}
$$

for a function $g_{5}\left(S^{1}, S^{2}\right)$ and the estimates of $\left|S^{*}(r \boldsymbol{e}(\theta))\right| \leq \frac{c_{3}^{\prime}}{\sqrt{1+r}} e^{-\alpha_{0} r},\left|\Phi_{3}^{*}(r \boldsymbol{e}(\theta))\right| \leq \frac{c_{3}^{*}}{\sqrt{1+r}} e^{-\alpha_{0} r}$ from (3.15) and (3.16) for positive constants $c_{3}^{\prime}$ and $c_{3}^{*}$, we can estimate

$$
\left|\left\langle g_{5}\left(S^{1}, S^{2}\right) S^{1}\left(S^{2}\right)^{2}, \Phi_{3}^{*}\left(z_{1}^{*}\right)\right\rangle_{2}\right| \leq c_{5} \int_{0}^{\infty} \frac{r}{1+r} e^{-2 \alpha_{0} r} \frac{1}{1+\left|z_{2}^{*}(r, \theta)\right|} e^{-2 \alpha_{0}\left|z_{2}^{*}(r, \theta)\right|} d r,
$$

where $z_{2}^{*}(r, \theta):=R_{1}(-\Xi)\left(r \boldsymbol{e}(\theta)-R_{1}\left(-\Theta_{1}\right) H\right)$. The estimate (3.17) for $\left|z_{2}^{*}(r, \theta)\right|$ gives

$$
\left|\left\langle g_{5}\left(S^{1}, S^{2}\right) S^{1}\left(S^{2}\right)^{2}, \Phi_{3}^{*}\left(z_{1}^{*}\right)\right\rangle_{2}\right| \leq O\left(e^{-2 \alpha_{0} h}\right)=O\left(\delta^{2}(h)\right) .
$$

Thus, we see

$$
b_{3}^{1}(\widetilde{y})=\left\langle k_{0} \gamma_{2}^{\prime}\left(S^{1}\right), S^{2}\right\rangle_{2}+\left\langle\left\{f_{0}^{\prime}\left(S^{1}\right)-f_{0}^{\prime}(0)\right\} S^{2}, \Phi_{3}^{*}\left(z_{1}^{*}\right)\right\rangle_{2}+O\left(\delta^{2}(h)\right) .
$$

Each term in (3.20) is computed as follows: Since $\left|z_{2}^{*}(r, \theta)\right|=h-r \cos \left(\theta-\Theta_{1}-\Theta_{H}\right)+O\left(\frac{1}{h}\right)$ and 


$$
\begin{aligned}
& S^{2}=S^{*}\left(z_{2}^{*}\right)=\frac{c_{0}^{\prime}}{\sqrt{\left|z_{2}^{*}\right|}} e^{-\alpha_{0}\left|z_{2}^{*}\right|}(1+O(\varepsilon)) \text { hold in the neighborhood of } \Omega_{1} \text {, Proposition } 2.5 \text { gives } \\
& \left\langle k_{0} \gamma_{2}^{\prime}\left(S^{1}\right), S^{2}\right\rangle_{2} \\
& =-\varepsilon \int_{0}^{2 \pi} \int_{0}^{\infty} b^{\prime}(\theta) r \xi\left(r-r_{0}\right) \gamma_{2}^{\prime}\left(U_{0}(r)\right) \frac{c_{0}^{\prime}}{\sqrt{\left|z_{2}^{*}(r, \theta)\right|}} e^{-\alpha_{0}\left|z_{2}^{*}(r, \theta)\right|} d r d \theta+O\left(\varepsilon^{2} \delta(h)\right) \\
& =-\varepsilon \frac{c_{0}^{\prime} e^{-\alpha_{0} h}}{\sqrt{h}} \int_{0}^{2 \pi} \int_{0}^{\infty} \frac{1}{\sqrt{1+O\left(\frac{1}{h}\right)}} b^{\prime}(\theta) r \xi\left(r-r_{0}\right) \gamma_{2}^{\prime}\left(U_{0}(r)\right) \\
& \times e^{\alpha_{0} r \cos \left(\theta+\Theta_{1}-\Theta_{H}\right)}\left(1+O\left(\frac{1}{h}\right)\right) d r d \theta+O\left(\varepsilon^{2} \delta(h)\right) \\
& =\varepsilon \frac{m_{0} c_{0}^{\prime} e^{-\alpha_{0} h}}{\sqrt{h}} \int_{0}^{\infty} r \xi\left(r-r_{0}\right) \gamma_{2}^{\prime}\left(U_{0}(r)\right)\left(\int_{0}^{2 \pi} \sin m_{0} \theta e^{\alpha_{0} r \cos \left(\theta+\Theta_{1}-\Theta_{H}\right)} d \theta\right) d r \\
& \times\left(1+O\left(\frac{1}{h}\right)\right)+O\left(\varepsilon^{2} \delta(h)\right) \\
& =\varepsilon \frac{m_{0} c_{0}^{\prime} e^{-\alpha_{0} h}}{\sqrt{h}} \int_{0}^{\infty} r \xi\left(r-r_{0}\right) \gamma_{2}^{\prime}\left(U_{0}(r)\right)\left(\int_{0}^{2 \pi} \sin m_{0}\left(\theta-\Theta_{1}+\Theta_{H}\right) \theta e^{\alpha_{0} r \cos \theta} d \theta\right) d r \\
& \times\left(1+O\left(\frac{1}{h}\right)\right)+O\left(\varepsilon^{2} \delta(h)\right) \\
& =-\varepsilon \frac{m_{0} c_{0}^{\prime} e^{-\alpha_{0} h}}{\sqrt{h}} \sin m_{0}\left(\Theta_{1}-\Theta_{H}\right) \int_{0}^{\infty} r \xi\left(r-r_{0}\right) \gamma_{2}^{\prime}\left(U_{0}(r)\right)\left(\int_{0}^{2 \pi} \cos m_{0} \theta e^{\alpha_{0} r \cos \theta} d \theta\right) d r \\
& \times\left(1+O\left(\frac{1}{h}\right)\right)+O\left(\varepsilon^{2} \delta(h)\right),
\end{aligned}
$$

that is, we have

$$
\left\langle k_{0} \gamma_{2}^{\prime}\left(S^{1}\right), S^{2}\right\rangle_{2}=\frac{\varepsilon}{\sqrt{h}} e^{-\alpha_{0} h} N_{m_{0}}^{\prime} \sin m_{0}\left(\Theta_{1}-\Theta_{H}\right)\left(1+O\left(\frac{1}{h}+\varepsilon\right)\right),
$$

where $N_{m_{0}}^{\prime}:=-m_{0} c_{0}^{\prime} \int_{0}^{\infty} r \xi\left(r-r_{0}\right) \gamma_{2}^{\prime}\left(U_{0}(r)\right)\left(\int_{0}^{2 \pi} \cos m_{0} \theta e^{\alpha_{0} r \cos \theta} d \theta\right) d r$. 
On the other hand, it follows that

$$
\begin{aligned}
& \left\langle\left\{f_{0}^{\prime}\left(S^{1}\right)-f_{0}^{\prime}(0)\right\} S^{2}, \Phi_{3}^{*}\left(z_{1}^{*}\right)\right\rangle_{2} \\
& =\varepsilon \int_{0}^{\infty} \int_{0}^{2 \pi} r\left\{f_{0}^{\prime}\left(U_{0}(r)\right)-f_{0}^{\prime}(0)\right\} U_{0}\left(\left|z_{2}^{*}(r, \theta)\right|\right) V_{1}^{*}(r) \sin m_{0} \theta d \theta d r+O\left(\varepsilon^{2} \delta(h)\right) \\
& =\varepsilon \int_{0}^{\frac{3}{4} h} \int_{0}^{2 \pi} r\left\{f_{0}^{\prime}\left(U_{0}(r)\right)-f_{0}^{\prime}(0)\right\} U_{0}\left(\left|z_{2}^{*}(r, \theta)\right|\right) V_{1}^{*}(r) \sin m_{0} \theta d \theta d r \\
& +\varepsilon \int_{\frac{3}{4} h}^{\infty} \int_{0}^{2 \pi} r\left\{f_{0}^{\prime}\left(U_{0}(r)\right)-f_{0}^{\prime}(0)\right\} U_{0}\left(\left|z_{2}^{*}(r, \theta)\right|\right) V_{1}^{*}(r) \sin m_{0} \theta d \theta d r+O\left(\varepsilon^{2} \delta(h)\right) \\
& =\varepsilon \int_{0}^{\frac{3}{4} h} \int_{0}^{2 \pi} r\left\{f_{0}^{\prime}\left(U_{0}(r)\right)-f_{0}^{\prime}(0)\right\} \frac{c_{0}^{\prime}}{\sqrt{\left|z_{2}^{*}\right|}} e^{-\alpha_{0}\left|z_{2}^{*}\right|} V_{1}^{*}(r) \sin m_{0} \theta d \theta d r \\
& +\varepsilon \int_{\frac{3}{4} h}^{\infty} \int_{0}^{2 \pi} r\left\{f_{0}^{\prime}\left(U_{0}(r)\right)-f_{0}^{\prime}(0)\right\} U_{0}\left(\left|z_{2}^{*}(r, \theta)\right|\right) V_{1}^{*}(r) \sin m_{0} \theta d \theta d r+O\left(\varepsilon^{2} \delta(h)\right) \\
& =\varepsilon \int_{0}^{\frac{3}{4} h} \int_{0}^{2 \pi} r\left\{f_{0}^{\prime}\left(U_{0}(r)\right)-f_{0}^{\prime}(0)\right\} \frac{c_{0}^{\prime}}{\sqrt{\left|z_{2}^{*}\right|}} e^{-\alpha_{0}\left|z_{2}^{*}\right|} V_{1}^{*}(r) \sin m_{0} \theta d \theta d r \\
& +\varepsilon \int_{\frac{3}{4} h}^{\infty} O\left(e^{-2 \alpha_{0} r}\right) d r+O\left(\varepsilon^{2} \delta(h)\right) \\
& =\varepsilon \frac{c_{0}^{\prime}}{\sqrt{h}} e^{-\alpha_{0} h} \int_{0}^{\frac{3}{4} h} \int_{0}^{2 \pi} \frac{r\left\{f_{0}^{\prime}\left(U_{0}(r)\right)-f_{0}^{\prime}(0)\right\} V_{1}^{*}(r) \sin m_{0} \theta}{\sqrt{1-\frac{r}{h} \cos \left(\theta+\Theta_{1}-\Theta_{H}\right)+O\left(\frac{r^{2}}{h^{2}}\right)}} e^{\alpha_{0} r \cos \left(\theta+\Theta_{1}-\Theta_{H}\right)+O\left(\frac{r^{2}}{h}\right)} d \theta d r \\
& +O\left(\varepsilon e^{-\frac{3}{2} \alpha_{0} h}+\varepsilon^{2} \delta(h)\right) \\
& =\varepsilon \frac{c_{0}^{\prime}}{\sqrt{h}} e^{-\alpha_{0} h} \int_{0}^{\frac{3}{4} h} \int_{0}^{2 \pi} r\left\{f_{0}^{\prime}\left(U_{0}(r)\right)-f_{0}^{\prime}(0)\right\} V_{1}^{*}(r) \sin m_{0} \theta e^{\alpha_{0} r \cos \left(\theta+\Theta_{1}-\Theta_{H}\right)}\left(1+O\left(\frac{r^{2}}{h}\right)\right) d \theta d r \\
& +O\left(\varepsilon e^{-\frac{3}{2} \alpha_{0} h}+\varepsilon^{2} \delta(h)\right) \\
& =\varepsilon \frac{c_{0}^{\prime}}{\sqrt{h}} e^{-\alpha_{0} h}\left(\int_{0}^{\frac{3}{4} h} \int_{0}^{2 \pi} r\left\{f_{0}^{\prime}\left(U_{0}(r)\right)-f_{0}^{\prime}(0)\right\} V_{1}^{*}(r) \sin m_{0}\left(\theta-\Theta_{1}+\Theta_{H}\right) e^{\alpha_{0} r \cos \theta} d \theta d r\right. \\
& \left.+\frac{1}{h} \int_{0}^{\frac{3}{4} h} O\left(r^{2} e^{-2 \alpha_{0} r}\right) d r\right)+O\left(\varepsilon e^{-\frac{3}{2} \alpha_{0} h}+\varepsilon^{2} \delta(h)\right) \\
& =-\varepsilon \frac{c_{0}^{\prime}}{\sqrt{h}} e^{-\alpha_{0} h} \int_{0}^{\infty} \int_{0}^{2 \pi} r\left\{f_{0}^{\prime}\left(U_{0}(r)\right)-f_{0}^{\prime}(0)\right\} V_{1}^{*}(r) \cos m_{0} \theta e^{\alpha_{0} r \cos \theta} d \theta d r \sin m_{0}\left(\Theta_{1}-\Theta_{H}\right) \\
& +O\left(\frac{\varepsilon}{h} \delta(h)+\varepsilon e^{-\frac{3}{2} \alpha_{0} h}+\varepsilon^{2} \delta(h)\right) .
\end{aligned}
$$

That is, we have

$$
\left\langle\left\{f_{0}^{\prime}\left(S^{1}\right)-f_{0}^{\prime}(0)\right\} S^{2}, \Phi_{3}^{*}\left(z_{1}^{*}\right)\right\rangle_{2}=\frac{\varepsilon}{\sqrt{h}} e^{-\alpha_{0} h} N_{m_{0}}^{\prime \prime} \sin m_{0}\left(\Theta_{1}-\Theta_{H}\right)\left(1+O\left(\frac{1}{h}+\varepsilon+e^{-\alpha_{1} h}\right)\right)
$$

for $\alpha_{1}>0$, where

$$
N_{m_{0}}^{\prime \prime}:=-\int_{0}^{\infty} \int_{0}^{2 \pi} r\left\{f_{0}^{\prime}\left(U_{0}(r)\right)-f_{0}^{\prime}(0)\right\} V_{1}^{*}(r) \cos m_{0} \theta e^{\alpha_{0} r \cos \theta} d \theta d r .
$$


Thus, we see from (3.21) and (3.22)

$$
b_{3}^{1}(\widetilde{\boldsymbol{y}})=\frac{\varepsilon}{\sqrt{h}} e^{-\alpha_{0} h} N_{m_{0}} \sin m_{0}\left(\Theta_{1}-\Theta_{H}\right)\left(1+O\left(\frac{1}{h}+\varepsilon+e^{-\alpha_{1} h}\right)\right) .
$$

For $\Theta_{2}$, we have quite similar results as

$$
\left.b_{3}^{2} \widetilde{\boldsymbol{y}}\right)=\frac{\varepsilon}{\sqrt{h}} e^{-\alpha_{0} h} N_{m_{0}} \sin m_{0}\left(\Theta_{2}+\Theta_{H}\right)\left(1+O\left(\frac{1}{h}+\varepsilon+e^{-\alpha_{1} h}\right)\right) .
$$

Noting $\dot{\Theta}_{j}=\dot{\Xi}_{j}$, we complete the proof.

To check the theoretical results in Theorem 3.2 using real experiments in the following section, we consider the explicit motions of camphor particles by using the reduced equation

$$
\dot{\Xi}_{j}=\frac{\varepsilon}{\sqrt{h}} e^{-\alpha_{0} h} N_{m_{0}} \sin m_{0} \Xi_{j} .
$$

Proposition 3.7. If $f_{0}(u)$ is linear, then the constant $N_{m_{0}}$ is positive.

Proof. For $N_{m_{0}}=N_{m_{0}}^{\prime}+N_{m_{0}}^{\prime \prime}, N_{m_{0}}^{\prime \prime}=0$ holds by the definition of $N_{m_{0}}^{\prime \prime}$ and the linearity of $f_{0}(u)$. We will show the positivity of $N_{m_{0}}^{\prime}$. Since $\xi\left(r-r_{0}\right)$ is close to the Dirac $\delta$-function $\delta\left(r-r_{0}\right)$ with $\left|\int_{\mathbb{R}} g(r) \xi\left(r-r_{0}\right) d r-g\left(r_{0}\right)\right| \leq \delta\|g\|_{H^{1}(\mathbb{R})}$ for sufficiently small $\delta>0$ as stated in Section 2, we see

$$
\begin{aligned}
N_{m_{0}}^{\prime} & =-m_{0} c_{0}^{\prime} r_{0} \gamma_{2}^{\prime}\left(U_{0}\left(r_{0}\right)\right) \int_{0}^{2 \pi} \cos m_{0} \theta e^{\alpha_{0} r_{0} \cos \theta} d \theta+O(\delta) \\
& =-m_{0} c_{0}^{\prime} r_{0} \gamma_{2}^{\prime}\left(U_{0}\left(r_{0}\right)\right) \cdot 2 \pi I_{m_{0}}\left(\alpha_{0} r_{0}\right)+O(\delta) \\
& >0
\end{aligned}
$$

because $\gamma_{2}^{\prime}\left(U_{0}\left(r_{0}\right)\right)<0$ and $I_{m_{0}}\left(\alpha_{0} r_{0}\right)>0$ hold, where $I_{v}(z)$ is a modified Bessel function of the first kind with index $v$.

Thus, we can assume $N_{m_{0}}>0$ in natural situations as in $f_{0}(u)=-\alpha u$. Then (3.23) emphasizes that the angle $\boldsymbol{\Xi}_{j}$ of the camphor particle for the center line $\boldsymbol{p}_{2}-\boldsymbol{p}_{1}$ converges to $\pi / m_{0}$. Using the perturbation method under the assumption of sufficient distance between two particles, the consistent results were obtained, whose details are shown in Appendix A. In the following section, this result is checked in real experiments and numerical calculations for $m_{0}=2$ and 3 by fixing the barycenters of particles, which correspond to the case that $\gamma_{1}(u)=0$ in (3.1).

In this section, we focused on the dynamics of an angle of a non-radially symmetric camphor particle located sufficient far from the other particle. The dynamics of the translational motion was neglected by considering large $\tau_{1}$. The adjoint vectors with perturbative term were constructed using the adjoint vectors in the case of an isolated camphor particle. Then the time evolution equation for the angle of the camphor particle and its steady-state solution were derived as shown in Theorem 3.2, Eq. (3.23), and Proposition 3.7. 
(a)

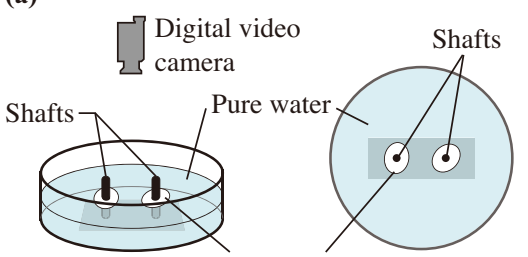

Elliptic camphor particle (b)

(b)

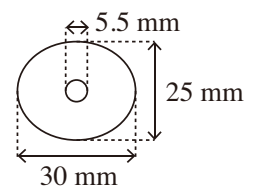

(c)

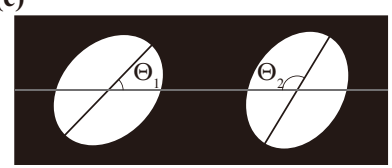

(d)

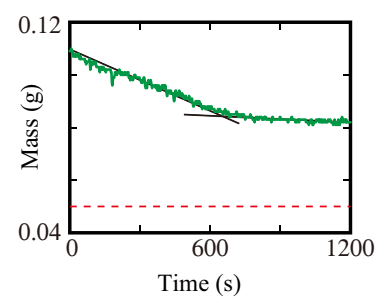

Figure 5: (a) Setup of the experiments. (b) Shape of the elliptic camphor particles. (c) Definition of the angles, $\Theta_{1}$ and $\Theta_{2}$, of the orientation of major axes of the elliptic camphor particles. (d) Time series of the mass of the elliptic filter paper after it was soaked in $0.3 \mathrm{~mol} / \mathrm{L}$ camphor methanol solution. The slope of the time series of the mass changed around $600 \mathrm{~s}$. The red broken line $(\sim 0.05 \mathrm{~g})$ shows the mass of the elliptic filter paper without soaking in camphor methanol solution.

\section{Experiments}

To confirm the analytical results, we performed experiments using a camphor-water system as shown in Fig. 5(a). The elliptic camphor particles were floated on pure water and made to interact with each other. The centers of mass of the elliptic camphor particles were fixed by the shafts made with a 3D printer (UP! Plus2, OPT Technologies, Japan), so that elliptic camphor particles could rotate freely. To prepare the elliptic camphor particles, first the filter paper (Whatman 1440-240, GE Healthcare Life Science, UK) was cut into an elliptic shape with a hole for the shaft at the center using a laser cutter (VLS 6.60, Universal Laser Systems, USA) as shown in Fig. 5(b). Then elliptic-shaped filter paper was soaked into a camphor methanol solution, which was prepared to be $0.3 \mathrm{~mol} / \mathrm{L}$ by putting camphor (Wako, Japan) into methanol (Wako, Japan) [25]. Then the elliptic filter paper was dried in air. The mass of the elliptic filter paper decreased because methanol and camphor evaporated into the air. The time series of the mass is shown in Fig. 5(d). The decreasing slope changed around $600 \mathrm{~s}$ after it had begun to be dried. The reason for the change in slope was that methanol in the filter paper had almost dried out, but camphor experiments, we thus remained since methanol evaporated faster than camphor. For the experiments, thus, we used the elliptic filter paper that had been dried for $600 \mathrm{~s}$. Pure water was prepared with a Millipore system (Elix UV3, Merck, Germany). The images were taken in the experiment using a digital video camera (IVIS HV30, Canon, Japan). All the experiments were performed at room temperature $\left(\sim 20^{\circ} \mathrm{C}\right)$. The obtained images were analyzed with image processing software (ImageJ, National Institutes of Health, USA). The orientations of the elliptic camphor particles in each frame of the movie were detected based on the following theoretical consideration.

The region corresponding to the elliptic camphor particle is denoted by $\hat{\Omega}_{\Theta}$ whose center of mass is $\left(x_{0}, y_{0}\right)$. Here $\Theta$ represents the orientation of the major axis of the ellipse. Then the second-order moments are defined by $\left\langle\left(x-x_{0}\right)^{2}\right\rangle_{\hat{\Omega}_{\theta}},\left\langle\left(y-y_{0}\right)^{2}\right\rangle_{\hat{\Omega}_{\Theta}}$, and $\left\langle\left(x-x_{0}\right)\left(y-y_{0}\right)\right\rangle_{\hat{\Omega}_{\Theta}}$, where

$$
\langle f(x, y)\rangle_{\hat{\Omega}_{\Theta}}=\int f(x, y) \chi_{\hat{\Omega}_{\Theta}}(x, y) d x d y,
$$



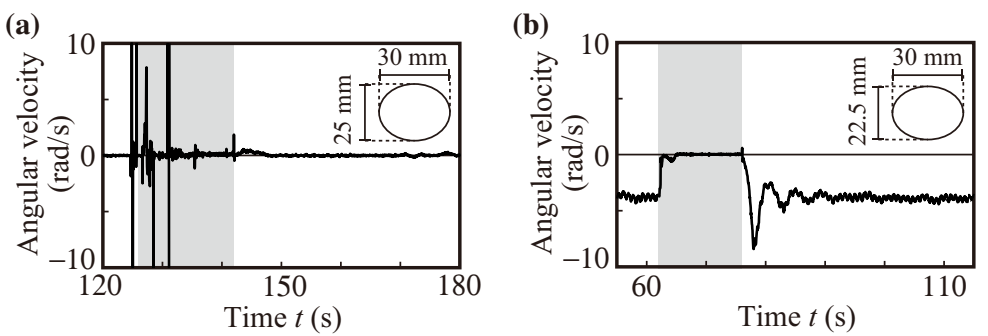

Figure 6: Time series of the angular velocity of the elliptic camphor particles floating on pure water. The centers of mass of the elliptic camphor particles were fixed by the shaft and its angular velocity was measured. The major axis of the ellipse was $30 \mathrm{~mm}$ and the minor axis of the ellipse was (a) $25 \mathrm{~mm}$ and (b) $22.5 \mathrm{~mm}$. The elliptic camphor particle exhibited (a) the rest state and (b) uniform rotation. To confirm the stability of the angular velocity, we added perturbations by poking the particle with tweezers. The time when the elliptic camphor particle was put onto the water surface was set as $t=0 \mathrm{~s}$. The periods while the elliptic camphor particles were perturbated are shown by shading with light gray in the plots. The angular velocity around $t=125 \mathrm{~s}$ in (a) was fluctuated due to the image of the apparatus for the perturbation, and the large absolute values of the angular velocities during the period of the perturbation were cut off to emphasize the difference in stationary angular velocity between the two conditions. Illustrations of the shape of elliptic camphor particles are shown in each plot as insets. Movies (fig6a.mpg for (a) and fig6b.mpg for (b)) are available in the Supplementary material (Appendix E).

and

$$
\chi_{\hat{\Omega}_{\Theta}}(x, y)= \begin{cases}1, & \text { if }(x, y) \in \hat{\Omega}_{\Theta}, \\ 0, & \text { if }(x, y) \notin \hat{\Omega}_{\Theta} .\end{cases}
$$

We can derive the relation between the second-order moments for the regions $\hat{\Omega} T$ heta and $\hat{\Omega}_{0}$ as

$$
\begin{aligned}
& \left(\begin{array}{cc}
\left\langle\left(x-x_{0}\right)^{2}\right\rangle_{\hat{\Omega}_{\Theta}} & \left\langle\left(x-x_{0}\right)\left(y-y_{0}\right)\right\rangle_{\hat{\Omega}_{\Theta}} \\
\left\langle\left(x-x_{0}\right)\left(y-y_{0}\right)\right\rangle_{\hat{\Omega}_{\Theta}} & \left\langle\left(y-y_{0}\right)^{2}\right\rangle_{\hat{\Omega}_{\Theta}}
\end{array}\right)\left(\begin{array}{cc}
\cos \Theta & -\sin \Theta \\
\sin \Theta & \cos \Theta
\end{array}\right) \\
& =\left(\begin{array}{cc}
\cos \Theta & -\sin \Theta \\
\sin \Theta & \cos \Theta
\end{array}\right)\left(\begin{array}{cc}
\left\langle\left(x-x_{0}\right)^{2}\right\rangle_{\hat{\Omega}_{0}} & 0 \\
0 & \left\langle\left(y-y_{0}\right)^{2}\right\rangle_{\hat{\Omega}_{0}}
\end{array}\right),
\end{aligned}
$$

where $\hat{\Omega}_{0}$ is the region of a camphor particle located at $\left(x_{0}, y_{0}\right)$ with the characteristic angle $\Theta=0$. Here $\left\langle\left(x-x_{0}\right)^{2}\right\rangle_{\hat{\Omega}_{\Theta}},\left\langle\left(y-y_{0}\right)^{2}\right\rangle_{\hat{\Omega}_{\Theta}}$, and $\left\langle\left(x-x_{0}\right)\left(y-y_{0}\right)\right\rangle_{\hat{\Omega}_{\Theta}}$ were obtained for each frame in the movies. By solving equation (4.26) as an eigenvalue problem, we obtained the orientations of the particles for each frame [38].

Before we observed the interaction of two elliptic camphor particles, we checked whether a single elliptic camphor particle rotates spontaneously. We prepared two kinds of elliptic camphor particles with different shapes and measured the angular velocity of the elliptic camphor particles. The elliptic camphor particle with aspect ratio much greater than one (elongated shape) exhibited uniform rotation but the particle with the aspect ratio around 1 (almost circular shape) exhibited rest at an arbitrary orientation as shown in Fig. 6.

Then, we performed the experiment for interacting elliptic camphor particles. Hereafter we used camphor particles whose major and minor axes are $30 \mathrm{~mm}$ and $25 \mathrm{~mm}$, which did not rotate 

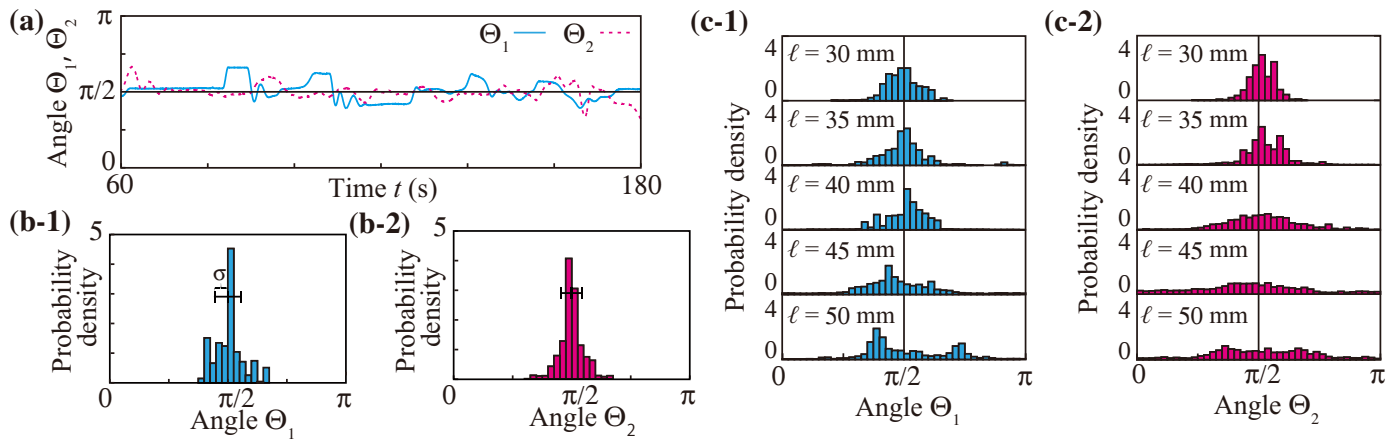

Figure 7: Experimental results for two interacting camphor particles. (a) Time series of the orientation of the elliptic camphor particles when $\ell=30 \mathrm{~mm}$. The time when the elliptic camphor particles were put onto the water surface was set as $t=0 \mathrm{~s}$. (b) Probability density of (b-1) $\Theta_{1}$ and (b-2) $\Theta_{2}$ corresponding to the time series shown in (a). (c) Probability density of (c-1) $\Theta_{1}$ and (c-2) $\Theta_{2}$ for each distance between the centers of mass, $\ell$. The histograms in (c) are obtained by summarizing the data of more than three trials for each $\ell$.

in an isolated condition. Time was set to be zero when the elliptic camphor particles were put onto pure water. We used the data from $60 \mathrm{~s}$ to $180 \mathrm{~s}$ to avoid the effect of the initial fluctuation. The time series of the orientations are shown in Fig. 7(a). Here, the orientations of the two elliptic camphor particles are denoted as $\Theta_{1}$ and $\Theta_{2}\left(\Theta_{1}, \Theta_{2} \in[0, \pi)\right)$ as shown in Fig. 5(c). The probability density for $\Theta_{1}$ and $\Theta_{2}$ is shown in Fig. 7(b-1) and (b-2), respectively. It can be seen that both of the probability densities had peaks around $\Theta_{1}=\pi / 2$ and $\Theta_{2}=\pi / 2$. These results mean that the major axes of the elliptic camphor particles were perpendicular to the line that connected the centers of mass, and were consistent with the analytical results. We also observed the effects of the distance between the center of mass, $\ell$. We performed the experiments for $\ell=30,35,40,45$, and $50 \mathrm{~mm}$. The results are shown in Fig. 7(c). The orientations tended to align for smaller $\ell$. To confirm the stability of the rest state, $\Theta_{1}=\Theta_{2}=\pi / 2$, we measured the orientations of the elliptic camphor particles after perturbation. We added the perturbation in two ways: (a) the fixed elliptic camphor particles in arbitrary orientations were released at the same time and (b) one of the elliptic camphor particles was poked by tweezers. The time series of the orientations and the snapshots are shown in Fig. 8. In both cases, the orientation of the elliptic camphor particles converged to $\Theta_{1}=\Theta_{2}=\pi / 2$ around $20 \mathrm{~s}$ after the perturbation had ceased. Thus, it was concluded that the rest state was stable.

\section{Numerical calculation}

To confirm the validity of the analytical results, we also performed numerical calculation. In the numerical calculation, we consider a two-dimensional concentration field, $u$, of size $L_{x} \times L_{y}$. The field size was taken large enough so that boundaries did not significantly affect the motion of the camphor particle. The time evolution equation for $u$ is written as

$$
\partial_{t} u=d \Delta u-\alpha u+f\left(x, y, \Theta_{c}\right),
$$

where $f\left(x, y, \Theta_{\mathrm{c}}\right)$ is $a_{0}(>0)$ inside the camphor particle and 0 outside of it. In the numerical calculation, equation (5.27) was discretized with the mesh size, $\Delta x=1$, and time step, $\Delta t=0.01$, 

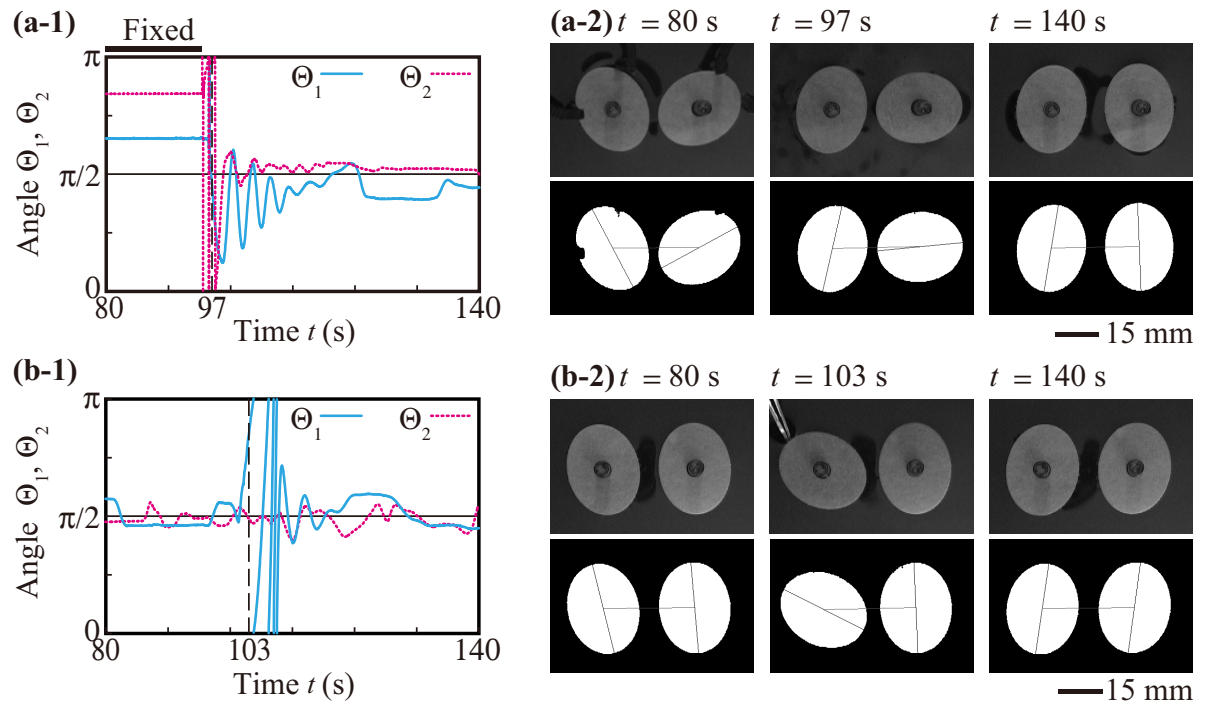

Figure 8: Time series of the orientations and snapshots of the elliptic camphor particles. The time when the elliptic camphor particles were put onto the water surface was set as $t=0 \mathrm{~s}$. (a) The case when the elliptic camphor particles fixed in arbitrary orientations were released at the same time $(t=97 \mathrm{~s})$. (b) The case when one of the elliptic camphor particles was poked by tweezers $(t=103 \mathrm{~s})$. (a-1,b-1) Time series of the orientation. (a-2,b-2) Snapshots of the elliptic camphor particles (upper) and their binarized images (lower). The distance between the centers of mass was $\ell=30 \mathrm{~mm}$ in both cases. Movies (fig8a.mpg for (a) and fig8b.mpg for (b)) are available in the Supplementary material (Appendix E). 
and was solved with the explicit Euler method. We took the Neumann boundary condition at the boundary of the calculation field. The time evolution equation for the characteristic angle $\Theta_{c}$ is written down as

$$
I \ddot{\Theta}_{\mathrm{c}}=-\eta \dot{\Theta}_{\mathrm{c}}+T,
$$

where $I$ is the moment of inertia of the camphor particle and $\eta$ is the coefficient of the friction force originating from the viscosity. Here, $T$ is the torque working on the camphor particle, which is calculated by the summation of the torque originating from the surface tension at discrete points $R(\Theta) \tilde{\boldsymbol{r}}_{i}(i=1,2, \ldots, N)$. In addition, $R(\Theta)$ denotes the rotation matrix in two dimensions,

$$
R(\Theta)=\left(\begin{array}{cc}
\cos \Theta & -\sin \Theta \\
\sin \Theta & \cos \Theta
\end{array}\right),
$$

and the vectors $\tilde{\boldsymbol{r}}_{i}$ correspond to the positions of the points along the periphery of the camphor particles. We set $\tilde{\boldsymbol{r}}_{i}$ so that the arc lengths between the neighboring points are equal to each other. To obtain these points, we first calculated the total peripheral length, $\Lambda$, of the camphor particles by numerical integration, and then numerically obtained the positions of the point on the periphery so that the arc length from the neighboring point was equal to $\Lambda / N$. Here, we set $N=200$. The time evolution equation for $\Theta_{\mathrm{c}},(5.28)$, was also solved with the Euler method with the same time step $\Delta t=0.01$. In this calculation, we do not consider the motion of the center of mass of the camphor particle, and the center of mass of the particle is always located at the center of the calculation field.

The torque was calculated as

$$
T=\sum_{i=1}^{N} \gamma\left(u\left(\boldsymbol{r}_{\mathrm{c}}+R(\Theta) \tilde{\boldsymbol{r}}_{i}\right)\right)\left(x_{i} n_{y}\left(x_{i}, y_{i}\right)-y_{i} n_{x}\left(x_{i}, y_{i}\right)\right),
$$

where $\tilde{\boldsymbol{r}}_{i}=\left(x_{i}, y_{i}\right)$ and $\boldsymbol{n}(x, y)=\left(n_{x}(x, y), n_{y}(x, y)\right)$ is the normal vector at the periphery of the ellipse $(x, y)$. Here $\gamma(u)$ should be a decreasing function of $u$ and we assumed that

$$
\gamma(u)=\gamma_{0}-k u,
$$

where $k$ and $\gamma_{0}$ are positive constants. It should be noted that $\gamma_{0}$ corresponds to the surface tension of pure water and that it does not affect the results.

As for the shape of the camphor particle, we considered an elliptic camphor particle. The shape of the camphor particle when $\Theta_{c}=0$ is described as

$$
\frac{x^{2}}{x_{0}^{2}}+\frac{y^{2}}{y_{0}^{2}}=1
$$

where $x_{0}$ and $y_{0}$ are the lengths of the major and minor axes, where $x_{0}>y_{0}>0$. In this calculation, we set $x_{0}=42$ and $y_{0}=35$, so that the aspect ratio corresponds to the condition in the experiments. The calculation was started with small angular velocity, and then small perturbation was added at $t=200$ to avoid remaining at unstable states. The angular velocity at $t=2000$ was adopted to the saturated value of it. The parameters were set as $d=10, \alpha=0.1$, $k=0.00001, a_{0}=1, I=0.01$, and $L_{x}=L_{y}=512$.

In Fig. 9, the behavior of a single camphor particle is shown. In Fig. 9(a) and (b), time series of the angular velocity are shown. The angular velocity converged to zero when $\eta$ was larger as in Fig. 9(a) and it converged to a constant nonzero value when $\eta$ was smaller as in Fig. 9(b). 


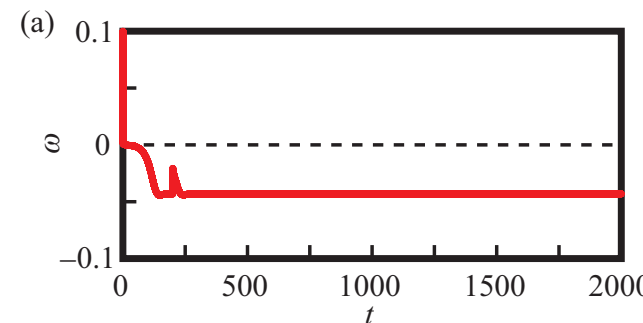

(c)
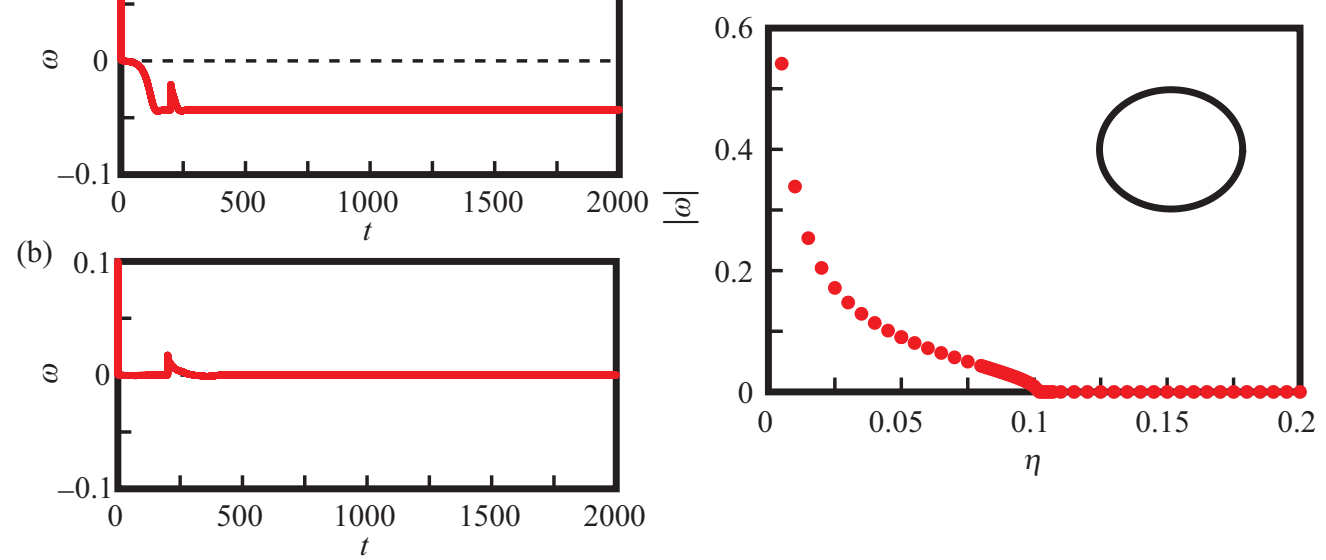

Figure 9: Results of the numerical calculation for a single elliptic camphor particle. (a) Time series of the angular velocity $\omega$ when $\eta=0.08$. The camphor particle kept rotating with a constant angular velocity even after a long duration. (b) Time series of the angular velocity $\omega$ when $\eta=0.12$. The camphor particle stopped after a long time. (c) Bifurcation diagram for the angular velocity $|\omega|$ when $\eta$ was changed. The camphor particle stopped after a long time for $\eta>\eta_{\text {th }} \simeq 0$. 1 , while it exhibited rotational motion at a constant angular velocity for $\eta<\eta_{\text {th }}$. Illustration of the shape of an elliptic camphor particle is shown as an inset. Movies (fig9a.mpg for (a) and fig9b.mpg for (b)) are available in the Supplementary material (Appendix E).

By scanning $\eta$, we obtained the bifurcation diagram for the angular velocity as in Fig. 9(c). The converged angular velocity was zero when $\eta$ was larger than the threshold value $\eta_{\text {th }}$, while it converged to a nonzero value when $\eta$ was smaller than $\eta_{\text {th }}$. It can be said that supercritical pitchfork bifurcation occurs at $\eta=\eta_{\text {th }}$.

Hereafter, we adopted $\eta=0.12$, which is larger than $\eta_{\text {th }}$. This means that a single camphor particle could not move spontaneously in this condition. Then, we investigated the interaction between two elliptic camphor particles. The distance between the centers of mass of the camphor particles were set as $\ell$. In this case, we adopted the same parameters as in the single particle case, except for $L_{x}$ and $L_{y}$. We set $L_{x}=1024$ and $L_{y}=512$ for this calculation, and the center of the two camphor particles was set at the center of the calculation field. Just like the single-particle case, the calculation was started with small angular velocities and small perturbation was added at $t=1000$. Then, we performed calculations until $t=2000$. The angles $\Theta_{1}$ and $\Theta_{2}$ are defined as in Fig. 5.

The results of the time series of $\Theta_{1}$ and $\Theta_{2}$ are shown in Fig. 10. When $\ell$ was smaller, both $\Theta_{1}$ and $\Theta_{2}$ converged to $\pi / 2$ as in Fig. 10(a). The results well correspond to the analytical and experimental results. When $\ell$ was larger, there was little correlation between $\Theta_{1}$ and $\Theta_{2}$. From the analytical results, for any $\ell$, the major axes should be aligned perpendicular to the line connecting the particle centers if there is no noise. We performed the numerical calculation with different mesh size $\Delta x$, and exemplified that this discrepancy comes from the discretization in the numerical calculation. The details are in Appendix B.

To examine the robustness against noise, we also performed numerical calculation by explic- 


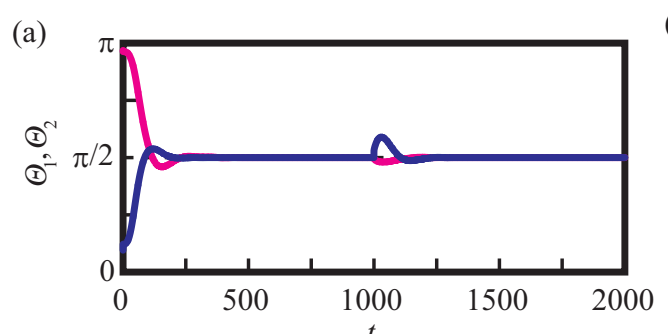

(c)
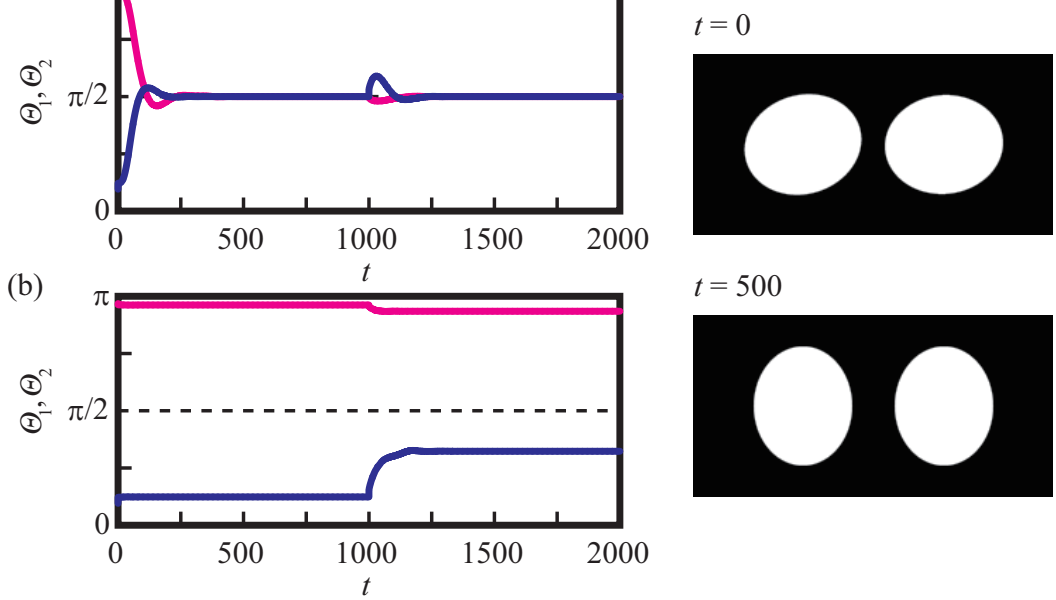

$t=500$

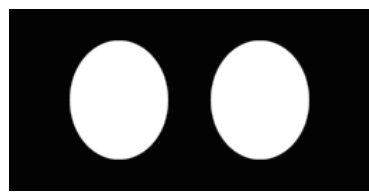

Figure 10: Results of numerical calculation for the two interacting elliptic camphor particles. The parameter for friction constant $\eta$ was set as $\eta=0.12$, so that a single particle did not keep moving but stopped. (a) Time series of the angles $\Theta_{1}$ and $\Theta_{2}$ for $\ell=100$. $\Theta_{1}$ and $\Theta_{2}$ converged to $\pi / 2$, which means that two elliptic camphor particles were aligned by the interaction. (b) Time series of the angles $\Theta_{1}$ and $\Theta_{2}$ for $\ell=160$. There was no correlation between $\Theta_{1}$ and $\Theta_{2}$. Blue (dark gray) and magenta (light gray) curves show $\Theta_{1}$ and $\Theta_{2}$, respectively. (c) Snapshots of the motion of two elliptic camphor particles for $\ell=100$, corresponding to (a). The time $t$ corresponds to the time $t$ in (a). A movie (fig10a.mpg for (a)) is available in the Supplementary material (Appendix E).

itly adding the noise to equation. (5.28) as

$$
I \ddot{\Theta}_{\mathrm{c}}=-\eta \dot{\Theta}_{\mathrm{c}}+T+\zeta(t)
$$

where $\zeta(t)$ is white Gaussian noise obeying

$$
\langle\zeta(t)\rangle=0,
$$

and

$$
\langle\zeta(t) \zeta(s)\rangle=2 \sigma^{2} \delta(t-s),
$$

where $\sigma=1$ and $\delta(\cdot)$ is the Dirac's delta function.

We changed $\ell$ and ran the numerical calculations from 64 different initial conditions $\left(\Theta_{1}=\right.$ $\left.m \pi / 4, \Theta_{2}=n \pi / 4, m, n \in \mathbb{Z}, 0 \leq m \leq 7,0 \leq n \leq 7\right)$ for each $\ell$. Using $\Theta_{1}$ and $\Theta_{2}$ from $t=200$ to 700 with time interval of 1 , we obtained histograms as shown in Fig. 11. For small $\ell$, the probability density on $\Theta$ has a peak around $\Theta=\pi / 2$, which corresponds to the parallel alignment of the major axes of the two camphor particles. The peak became broader as $\ell$ increases. For $\ell>140$, the probability density was almost uniform though there is fluctuation originating from the noise. From these results, the features of the histogram observed in experiments shown in Fig. 7 is considered to originate from the competition between the interaction and fluctuation.

We also performed numerical calculations on the motion of three-mode shaped camphor particles to evaluate the validity of the analytical results. Here, we consider a camphor particle whose shape is described as

$$
r(\theta)=r_{0}+r_{3} \cos 3 \theta,
$$




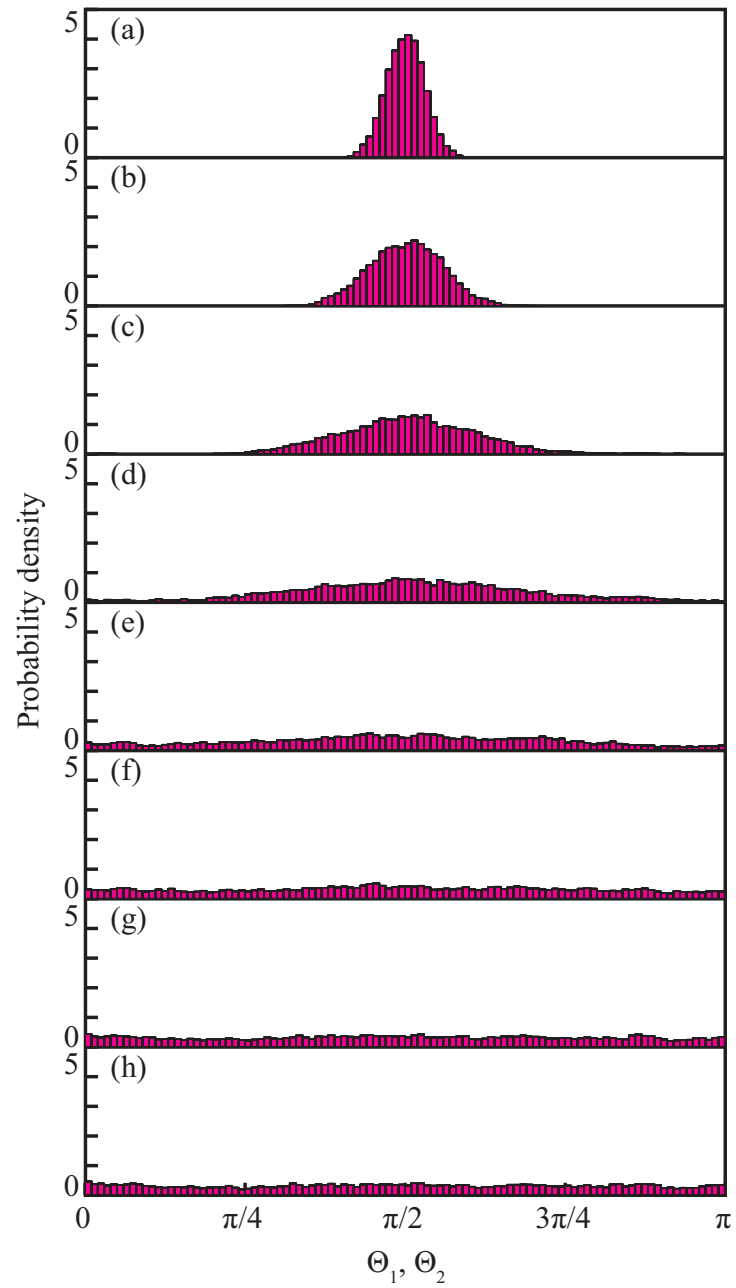

Figure 11: Probability density of $\Theta$ by the numerical calculation with noise. The distance between two elliptic camphor particles $\ell$ was varied: (a) $\ell=90$, (b) $\ell=100$, (c) $\ell=110$, (d) $\ell=120$, (e) $\ell=130$, (f) $\ell=140$, (g) $\ell=150$, and (f) $\ell=160$. For small $\ell$, the probability density has a peak around $\pi / 2$. 


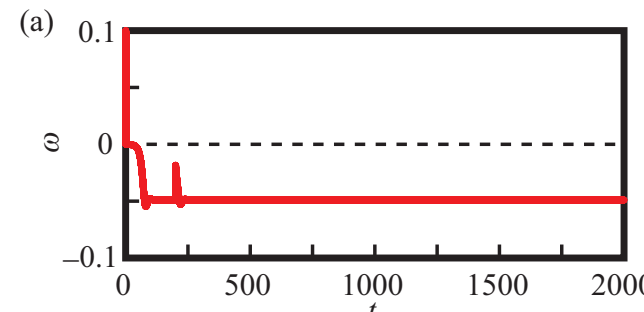

(c)

(b)
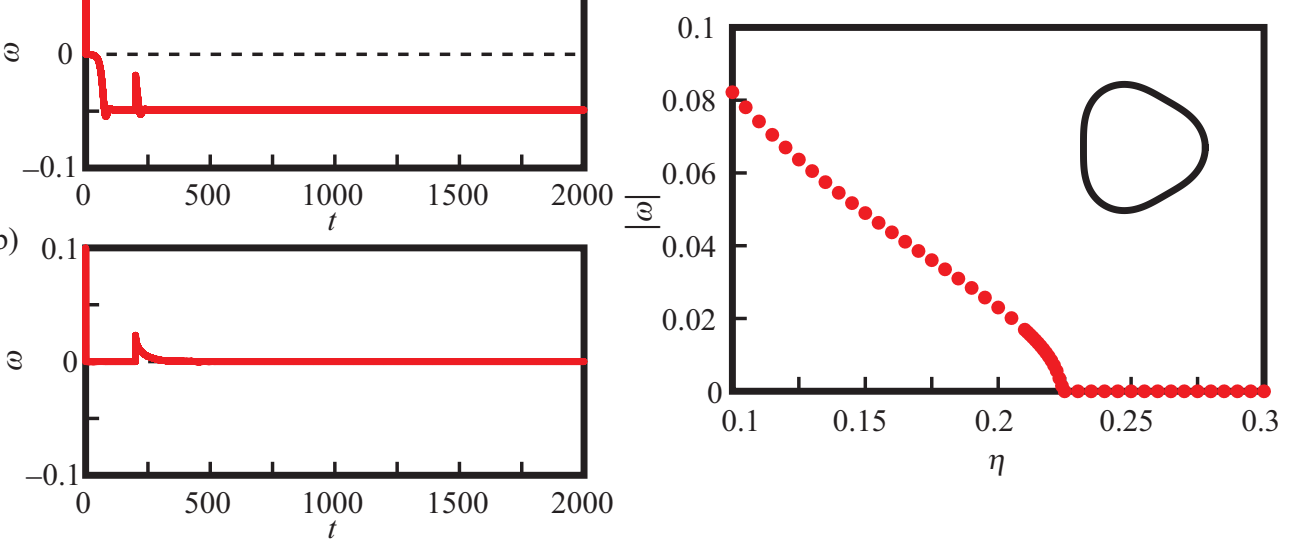

Figure 12: Results of numerical calculation for a single three-mode shaped camphor particle. (a) Time series of the angular velocity $\omega$ when $\eta=0.15$. The camphor particle kept rotating with a constant angular velocity $\omega$ after a long time. (b) Time series of the angular velocity when $\eta=0.25$. The camphor particle stopped after a long time. (c) Bifurcation diagram for the angular velocity $|\omega|$ when $\eta$ was varied. The camphor particle stopped after a long time for $\eta>\eta_{\mathrm{th}}^{\prime} \simeq 0.225$, while it exhibited rotational motion at a constant angular velocity for $\eta<\eta_{\mathrm{th}}^{\prime}$. Illustration of the shape of a three-mode shaped camphor particle is shown as an inset. Movies (fig12a.mpg for (a) and fig12b.mpg for (b)) is available in the Supplementary material (Appendix E).

in the polar coordinates. Here, we adopted $r_{0}=40$ and $r_{3}=4$. The parameters except for those on the shape of the camphor particles were the same as those for the elliptic camphor particles. For the calculation of a single particle, we set $L_{x}=L_{y}=512$, and the center of mass of the particle was set at the field center. First, we examined the behavior of a single camphor particle. The results are shown in Fig. 12. In Fig. 12(a) and (b), time series of the angular velocity are shown. The angular velocity converged to zero when $\eta$ was larger as in Fig. 12(a) and it converged to a constant nonzero value when $\eta$ was smaller as in Fig. 12(b). By scanning $\eta$, we obtained the bifurcation diagram for the angular velocity as in Fig. 12. The converged angular velocity was zero when $\eta$ was larger than the threshold value $\eta_{\text {th }}^{\prime}$, while it converged to a nonzero value when $\eta$ was smaller than $\eta_{\mathrm{th}}^{\prime}$. These behaviors are similar to those of an elliptic camphor particle, and it can be said that supercritical pitchfork bifurcation occurs at $\eta=\eta_{\text {th }}^{\prime}$.

Therefore, we adopted $\eta=0.25$, which is larger than $\eta_{\mathrm{th}}^{\prime}$, i.e., a single camphor particle cannot move spontaneously in this condition. We investigated the interaction between two three-mode shaped camphor particles. The distance between the centers of mass of the camphor particles were set as $\ell$. In this case, we adopted the same parameters as in the single-particle case, except for $L_{x}$ and $L_{y}$. We set $L_{x}=1024$ and $L_{y}=512$ for this calculation, and the center of the two camphor particles was set at the center of the calculation field. Just like the single-particle case, the calculation was started with small angular velocities and small perturbation was added at $t=1000$. Then, we performed calculations until $t=2000$. The results of the time series of $\Theta_{1}$ and $\Theta_{2}$ are shown in Fig. 13, where the characteristic angles $\Theta_{1}$ and $\Theta_{2}\left(\Theta_{1}, \Theta_{2} \in[0,2 \pi / 3)\right)$ are defined as in Fig. 13(c). When $\ell$ was smaller, both $\Theta_{1}$ and $\Theta_{2}$ converged to $\pi / 3$ as in Fig. 13(a). In other words, the three-mode shaped particles tended to align with their flat sides facing each 

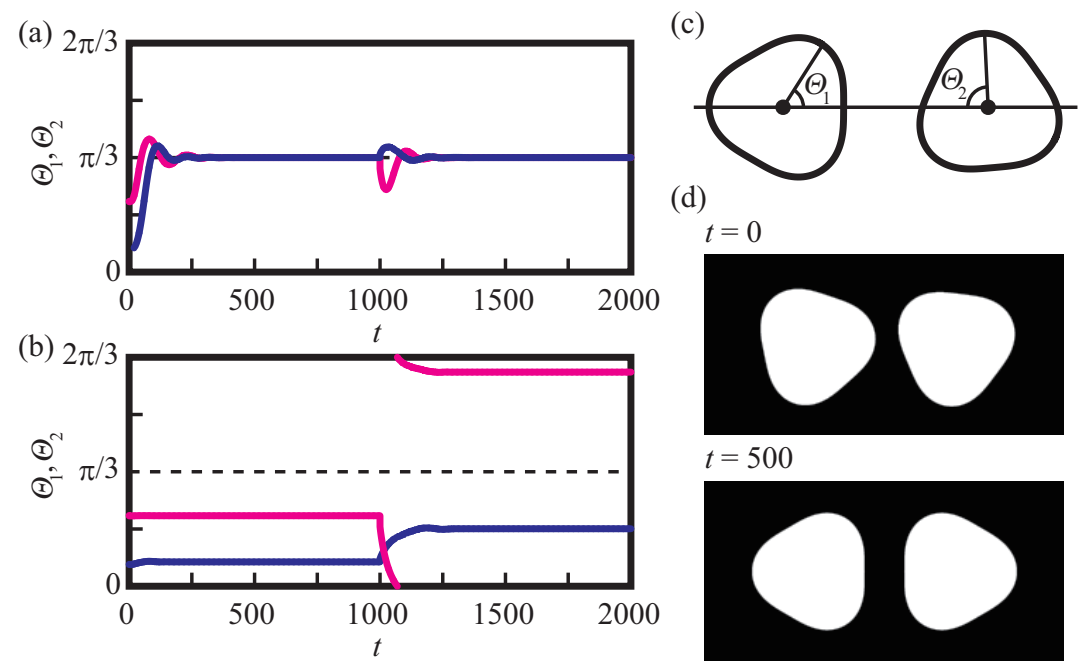

Figure 13: Results of numerical calculation for the two interacting three-mode shaped camphor particles. The parameter for friction constant $\eta$ was set as $\eta=0.25$, so that a single particle did not keep moving but stopped. (a) Time series of the angles $\Theta_{1}$ and $\Theta_{2}$ for $\ell=100$. Here $\Theta_{1}$ and $\Theta_{2}$ converged to $\pi / 3$, which means that two elliptic camphor particles were aligned by the interaction. (b) Time series of the angles $\Theta_{1}$ and $\Theta_{2}$ for $\ell=160$. There was no correlation between $\Theta_{1}$ and $\Theta_{2}$. Blue (dark gray) and magenta (light gray) curves show $\Theta_{1}$ and $\Theta_{2}$, respectively. (c) Definition of $\Theta_{1}$ and $\Theta_{2}$. (d) Snapshots of the motion of two elliptic camphor particles for $\ell=100$, corresponding to (a). The time $t$ corresponds to the time $t$ in (a). A movie (fig13a.mpg for (a)) is available in the Supplementary material (Appendix E).

other. The results well correspond to the analytical results. When $\ell$ was larger, there was little correlation between $\Theta_{1}$ and $\Theta_{2}$, which did not well correspond to the analytical prediction. This discrepancy can be explained in the same way as that for the two elliptic camphor particles.

In addition, we performed numerical calculation to confirm whether some hypotheses in theoretical part are satisfied. The details are shown in Appendix C. The theory suggests that the two interacting elliptic particles are aligned perpendicular to the line connecting their centers even when $f_{0}(u)$ includes the nonlinear decay term. This was also confirmed in the numerical calculation, whose results are shown in Appendix D.

\section{Conclusion}

In the present paper, the interaction between two non-radially symmetric camphor particles was discussed theoretically. Based on the model composed of the partial differential equation for the camphor concentration field together with the ordinary differential equations for the position and characteristic angle of the camphor particles, we obtained the ordinary differential equation describing the time evolution of the positions and characteristic angles by the center manifold reduction. It was shown theoretically that two interacting elliptic camphor particles should be aligned such that the major axes are parallel and perpendicular to the lines interconnecting the two particle centers. Such a prediction was confirmed by real experiments and numerical simulations. The main result (Theorem 3.1) can be applied not only for the camphor particles but for self-propelled particles interacting through a reaction-diffusion field. In fact, we only assume (H2) for $S^{*}(\boldsymbol{x})$ in the model equation (2.4) together with the monotone decrease of functions $\gamma_{1}$ 
and $\gamma_{2}$. That is, all necessary assumptions are only the very natural properties for $\gamma_{j}$ and the stability of the stationary spatial distribution of the surface concentration of the camphor molecular layer $u$ when a single camphor particle is spatially fixed, which is easily checked in the real experiment. Moreover, the equation of $u$ in (2.4) can be extended to the vector valued variable $\boldsymbol{u} \in \mathbb{R}^{N}$ for an arbitrary natural number $N$ and the same results as Theorems 3.1 and 3.2 can be obtained. Thus, the interacting motions of two camphor particles mentioned in this paper are expected to be universal. We hope the present approach will be extended to more complex systems.

\section{Acknowledgment}

The authors thank Natsuhiko Yoshinaga (Tohoku University, Japan) for his helpful discussion. This work was supported by JSPS KAKENHI Grants No. JP24340019, No. JP25103008, No. JP15K05199, No. JP16H03949, and No. JP17J05270. This work was supported by JST CREST Grants No. JPMJCR14D3 and No. JPMJCR15D2. This work was also supported by Cooperative Research Program of "Network Joint Research Center for Materials and Devices" (No. 2014007, No. 2015020, No. 20163002, No. 20165001, No. 20173002, and No. 20175002) to H. K. and Y. K., and the Sasakawa Scientific Research Grant from the Japan Science Society (No. 28-225) to Y. K.

[1] A. S. Mikhailov and V. Calenbuhr, From cells to societies: Models of complex coherent action, Springer, Berlin, 2002.

[2] S. J. Ebbens and J. R. Howse, In pursuit of propulsion at the nanoscale, Soft Matter 6 (2010) 726-738.

[3] T. Ohta, Dynamics of deformable active particles, J. Phys. Soc. Jpn. 86 (2017) 072001.

[4] J. D. Murray, Mathematical biology, Springer-Verlag, Berlin, 1993.

[5] C. P. Schenk, M. Or-Guil, M. Bode, and H.-G. Purwins, Interacting pulses in three-component reaction-diffusion systems on two-dimensional domains, Phys. Rev. Lett. 78 (1997) 3781-3784.

[6] Y. Nishiura, T. Teramoto, and K.-I. Ueda, Scattering and separators in dissipative systems, Phys. Rev. E 67 (2003) 056210 .

[7] S.-I. Ei, M. Mimura, and M. Nagayama, Interacting spots in reaction diffusion systems, Discrete Continuous Dyn. Sys. 14 (2006) 31-62.

[8] C. Tomlinson, On the motion of camphor on the surface water, Proc. R. Soc. London 11 (1860) 575-577.

[9] L. Rayleigh, Measurements of the amount of oil necessary in order to check the motions of camphor upon water, Proc. R. Soc. London 47 (1890) 364-367.

[10] S. Nakata, Y. Iguchi, S. Ose, M. Kuboyama, T. Ishii, and K. Yoshikawa, Self-rotation of a camphor scraping on water: New insight into the old problem, Langmuir 13 (1997) 4454-4458.

[11] Y. Hayashima, M. Nagayama, and S. Nakata, A camphor grain oscillates while breaking symmetry, J. Phys. Chem. B 105 (2001) 5353-5357.

[12] M. Nagayama, S. Nakata, Y. Doi, and Y. Hayashima, A theoretical and experimental study on the unidirectional motion of a camphor disk, Physica D 194 (2004) 151-165.

[13] X. Chen, S.-I. Ei, and M. Mimura, Self-motion of camphor discs - Model and analysis -, Networks Heterogeneous Media 4 (2009) 1-18.

[14] H. Kitahata, K. Iida, and M. Nagayama, Spontaneous motion of an elliptic camphor particle, Phys. Rev. E 87 (2013) 010901.

[15] K. Iida, H. Kitahata, and M. Nagayama, Theoretical study on the translation and rotation of an elliptic camphor particle, Physica D 272 (2014), 39-50.

[16] S. Nakata, M. Nagayama, H. Kitahata, N. J. Suematsu and T. Hasegawa, Physicochemical design and analysis of self-propelled objects that are characteristically sensitive to interfacial environments, Phys. Chem. Chem. Phys. 17 (2015) 10326-10338.

[17] Y. Koyano, T. Sakurai, and H. Kitahata, Oscillatory motion of a camphor grain in a one-dimensional finite region, Phys. Rev. E, 94, (2016) 042215.

[18] T. Miyaji, Arnold tongues in a billiard problem in nonlinear and nonequilibrium systems, Physica D 340 (2017) $14-25$.

[19] O. Schulz, M. Markus, Velocity distributions of camphor particle ensembles, J. Phys. Chem. B 111 (2007) 81758178 . 
[20] M. Mimura, T. Miyaji, O. Ohnishi, A billiard problem in nonlinear and nonequilibrium systems, Hiroshima Math. J. 37 (2007) 343-384.

[21] S. Soh, K. J. M. Bishop, B. A. Grzybowski, Dynamic self-assembly in ensembles of camphor boats, J. Phys. Chem. B 112 (2008) 10848-10853.

[22] N. J. Suematsu, S. Nakata, A. Awazu, H. Nishimori, Collective behavior of inanimate boats, Phys. Rev. E 81 (2010) 056210 .

[23] S. Soh, M. Branicki, B. A. Grzybowski, Swarming in shallow waters, J. Phys. Chem. Lett. 2 (2011) $770-774$.

[24] E. Heisler, N. J. Suematsu, A. Awazu, H. Nishimori, Swarming of self-propelled camphor boats, Phys. Rev. E 85 (2012) 055201

[25] Y. S. Ikura, E. Heisler, A. Awazu, H. Nishimori, S. Nakata, Collective motion of symmetric camphor papers in an annular water channel, Phys. Rev. E 88 (2013) 012911.

[26] K. Nishi, K. Wakai, T. Ueda, M. Yoshii, Y. S. Ikura, H. Nishimori, S. Nakata, M. Nagayama, Bifurcation phenomena of two self-propelled camphor disks on an annular field depending on system length, Phys. Rev. E 92 (2015) 022910 .

[27] N. J. Suematsu, K. Tateno, S. Nakata, H. Nishimori, Synchronized intermittent motion induced by the interaction between camphor disks, J. Phys. Soc. Jpn. 84 (2015) 034802.

[28] S.-I. Ei, K. Ikeda, M. Nagayama, A. Tomoeda, Reduced model from a reaction-diffusion system of collective motion of camphor boats, Discrete Continuous Dyn. Sys. Ser. S 8 (2015) 847-856.

[29] L. E. Scriven and C. V. Sternling, The Marangoni effects, Nature 187 (1960) 186-188.

[30] H. Kitahata, S.-i. Hiromatsu, Y. Doi, S. Nakata, and M. R. Islam, Self-motion of a camphor disk coupled with convection, Phys. Chem. Chem. Phys. 6 (2004) 2409-2414.

[31] E. Lauga and A. M. J. Davis, Viscous Marangoni propulsion, J. Fluid Mech. 705 (2012) 120-133.

[32] A. Wurger, Thermally driven Marangoni surfers J. Fluid Mech. 752 (2014) 589-601.

[33] H. Kitahata and N. Yoshinaga, Effective diffusion coefficient including the Marangoni effect, arXiv, (2016) 1604.01108 .

[34] A. Mikhailov and D. Meinköhn, Self-motion in physico-chemical systems far from thermal equilibrium, in L. Schimansky-Geier and T. Pöschel (Eds.) Stochastic Dynamics, Springer, Berlin, 1997.

[35] E. J. Banigan and J. F. Marko, Self-propulsion and interactions of catalytic particles in a chemically active medium, Phys. Rev. E 93 (2016) 012611.

[36] S.-I. Ei, The motion of weakly interacting pulses in reaction-diffusion systems, J. Dyn. Differ. Equ. 14 (2002), 85-137.

[37] S.-I. Ei, J. Wei, Dynamics of metastable localized patterns and its application to the interaction of spike solutions for the Gierer-Meinhardt systems in two spatial dimension, Jpn. J. Ind. Appl. Math. 19 (2002) 181-226.

[38] T. Banno, A. Asami, N. Ueno, H. Kitahata, Y. Koyano, K. Asakura, and T. Toyota, Deformable self-propelled micro-object comprising underwater oil droplets, Sci. Rep. 6 (2016) 31292.

\section{Appendix A. Calculation using the perturbation theorem}

Here, the dynamics of the two interacting camphor particles is discussed using the perturbation theorem. Here we assume the distance between two camphor particles is sufficiently large.

Now, we assume that one particle is located at $\boldsymbol{R}$. The stationary concentration field at $\boldsymbol{r}$ sufficiently far from the particle is described as

$$
u(\boldsymbol{r})=u_{0} \frac{\exp \left(-\sqrt{\frac{\alpha}{d}}|\boldsymbol{r}-\boldsymbol{R}|\right)}{\sqrt{|\boldsymbol{r}-\boldsymbol{R}|}},
$$

This description is obtained as follows; General solution of

$$
0=d \nabla^{2} u-\alpha u,
$$

is written as

$$
u(\boldsymbol{r})=\sum_{k=0}^{\infty}\left(A_{k} \cos k \theta+B_{k} \sin k \theta\right) I_{k}\left(\sqrt{\frac{\alpha}{d}} r\right)+\sum_{k=0}^{\infty}\left(C_{k} \cos k \theta+D_{k} \sin k \theta\right) \mathcal{K}_{k}\left(\sqrt{\frac{\alpha}{d}} r\right),
$$


where $r$ and $\theta$ are the polar coordinates in a two dimensional system. In Eq. (A.3), only $\mathcal{K}_{k}(r)$ does not diverge at $r \rightarrow \infty$. Considering the asymptotic form of the modified Bessel functions of the second kind, we obtain Eq. (A.1). Assuming that the distance between two particles is much greater than the radius of the particle, the concentration field near the particle originating from the other particle located at $\boldsymbol{R}=\boldsymbol{R} \boldsymbol{e}_{x}(R>0)$ is described as

$$
u(\boldsymbol{r})=u_{0} \frac{\exp \left(-\sqrt{\frac{\alpha}{d}}(R-x)\right)}{\sqrt{R-x}} .
$$

Then we consider a camphor particle whose center of mass is located at the origin. The shape of the particle is described in two-dimensional polar coordinates as

$$
r(\theta)=r_{0}+\epsilon \cos n(\theta-\Xi) .
$$

and we can calculate the arc-length unit $d \ell$ and normal vector $\boldsymbol{e}_{n}$ from the first order of $\epsilon$ as

$$
\begin{gathered}
d \ell=\left[r_{0}+\epsilon \cos n(\theta-\Xi)+O\left(\epsilon^{2}\right)\right] d \theta, \\
\boldsymbol{e}_{n}=\boldsymbol{e}_{r}+\epsilon n \sin n(\theta-\Xi) \boldsymbol{e}_{\theta}+O\left(\epsilon^{2}\right),
\end{gathered}
$$

where $\boldsymbol{e}_{r}$ and $\boldsymbol{e}_{\theta}$ are the unit vectors in two-dimensional polar coordinates.

Using these, we calculate the torque $T(\Xi)$ working on the camphor particle whose characteristic angle is $\Xi$ as

$$
T(\Xi)=\int_{0}^{2 \pi} \gamma(u(\boldsymbol{r}))\left(\boldsymbol{r} \times \boldsymbol{e}_{n}\right) \frac{d \ell}{d \theta} d \theta,
$$

where $\boldsymbol{r}=r(\theta) \boldsymbol{e}_{r}$.

For $n=2,3,4$, the torque is calculated in the approximation of $R \gg r_{0}$ as

$$
\begin{gathered}
T(\Xi)=\frac{u_{0} k \epsilon \pi}{8}\left(\frac{r_{0}}{R}\right)^{4} e^{-R} R^{\frac{3}{2}}\left(3+4 R+4 R^{2}\right) \sin 2 \Xi, \\
T(\Xi)=\frac{u_{0} k \epsilon \pi}{64}\left(\frac{r_{0}}{R}\right)^{5} e^{-R} R^{\frac{3}{2}}\left(15+18 R+12 R^{2}+8 R^{3}\right) \sin 3 \Xi, \\
T(\Xi)=\frac{u_{0} k \epsilon \pi}{768}\left(\frac{r_{0}}{R}\right)^{6} e^{-R} R^{\frac{3}{2}}\left(105+120 R+72 R^{2}+32 R^{3}+16 R^{4}\right) \sin 4 \Xi,
\end{gathered}
$$

respectively. These results meet the theoretical results in Eq. (3.23) and Proposition 3.7 with regard to the stable alignment of two interacting camphor particles.

\section{Appendix B. Numerical results with different spatial mesh size}

In Fig. 10, the alignment of two interacting camphor particles was perpendicular to the line connecting the centers of two particles for $\ell=100$, but not for $\ell=160$, where $\ell$ is the distance between two centers of camphor particles. In the theory, on the other hand, it should for any $\ell$. We assume that this discrepancy is due to the discretization in the numerical calculation. The concentration field decays exponentially depending on the distance, and thus interaction also decays exponentially. Therefore, it is natural that the numerical error exceeds the interaction for large $\ell$. Here, we performed the numerical calculation only by changing $\Delta x$ as $\Delta x=1$ and 

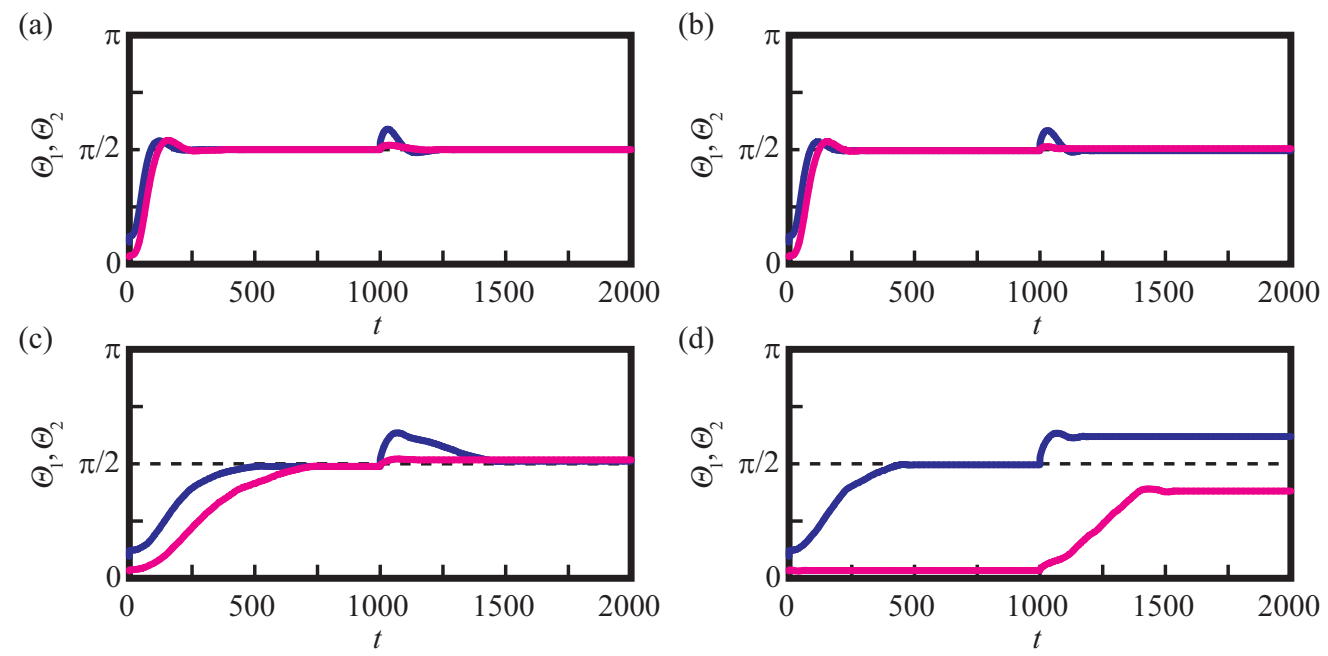

Figure B.14: Results of numerical calculation for the two interacting elliptic camphor particles by changing $\Delta x$ and $\ell$. The parameters are the same as those used in Fig. 10 except for $\ell$ and $\Delta x$. Time series of the angles $\Theta_{1}$ and $\Theta_{2}$ are shown. (a) $\ell=100$ and $\Delta x=1$. (b) $\ell=100$ and $\Delta x=2$. (c) $\ell=120$ and $\Delta x=1$. (d) $\ell=120$ and $\Delta x=2$.

$\Delta x=2$. The results are shown in Fig. B.14. As seen in Fig. B.14(a) and (b), the time evolution of $\Theta_{1}$ and $\Theta_{2}$ were almost the same for $\Delta x=1$ and $\Delta x=2$, and converged to $\pi / 2$ in both cases when $\ell=100$. On the contrary, when $\ell=120$, they converged to $\pi / 2$ for $\Delta x=1$ (see Fig. B.14(c)), while they did not converge to $\pi / 2$ for $\Delta x=2$ (see Fig. B.14(d)). This implies that the error originating from the discretization exceeded the interaction for $\Delta x=2$, but not for $\Delta x=1$, when $\ell=120$. This results support our suggestion on the origin of discrepancy.

\section{Appendix C. Confirmation of Hypotheses by numerical calculation}

In this section, we confirmed some of the hypotheses in theoretical part using numerical calculation. Hypotheses (H2), (H3) and (H4) are indirectly satisfied considering the results in Fig. 9. That is to say, there is a bifurcation point between the rest state and moving state as shown in Fig. 9, which means the rest state of the camphor particle can be realized for the finite value of $\eta$, i.e., the finite values of $\gamma_{1}$ and $\gamma_{2}$. We used $\eta=0.12$ for the numerical results shown in Figs. 10 to 11 . This means the condition used in our calculation satisfied (H2), (H3) and (H4).

Then, we show the radial profile of the stationary profile of the camphor concentration for a single camphor particle in Fig. C.15. As seen in Fig. C.15, the profiles decay exponentially, which satisfies the Hypothesis (H1) and the part of Hypothesis (H5).

\section{Appendix D. Results in the case that $f_{0}(u)$ is nonlinear}

In this section, we show the numerical results in the case that $f_{0}(u)$ is nonlinear, i.e., $f_{0}(u)=$ $-\alpha(1+\beta u) u$. We consider the time evolution equation for the concentration of camphor $u$ as

$$
\partial_{t} u=d \Delta u-\alpha(1+\beta u) u+f\left(x, y, \Theta_{\mathrm{c}}\right)
$$



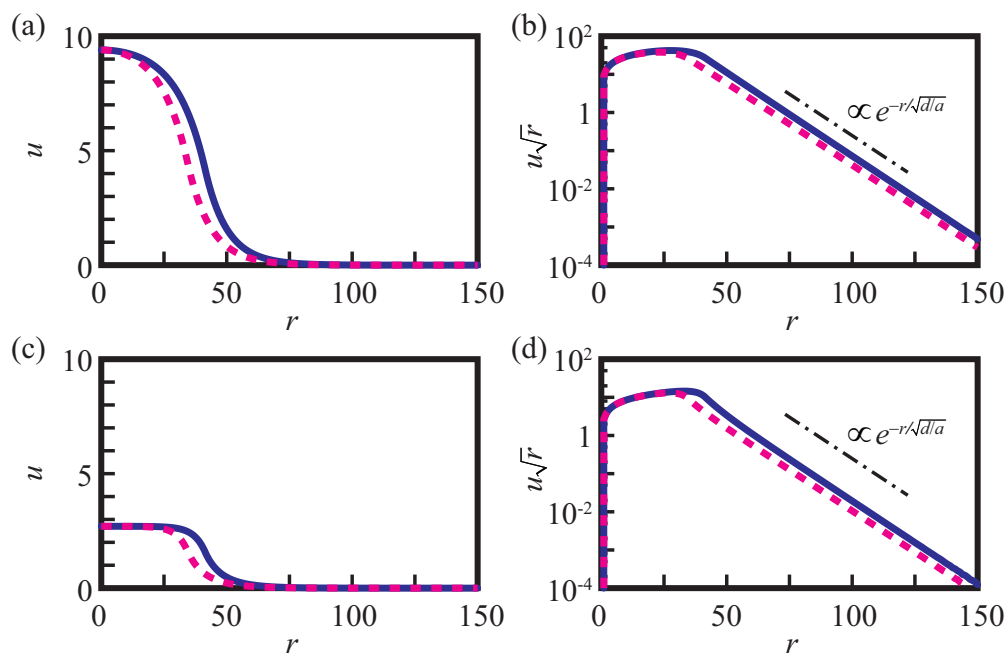

Figure C.15: Results of the numerical calculation for the radial profile of camphor concentration, $u$, for a single elliptic camphor particle. (a,b) The stationary profile for the system with the linear decay term in Eq. 5.27. (c,d) The stationary profile for the system with the nonlinear decay term in Eq. D.1. (a,c) Normal plot and (b,d) logarithmic plot are shown. The profiles in major-axis and minor-axis directions are shown with blue solid and magenta dashed curves, respectively. The parameters are the same as those in Fig. 9.

instead of Eq. (5.27). For the dynamics of a single elliptic particle used in Fig. 9, the bifurcation point shifted. It was around $\eta \simeq 0.017$ for $\beta=1$. The parameters are the same as those in Fig. 9 . The theory implies the two interacting elliptic camphor particles aligns in the same way as in the case that $f_{0}(u)=-\alpha u$. We performed numerical calculation for the interacting two elliptic camphor particles. The result is shown in Fig. D.16. The major axes were aligned perpendicular to the line connecting the particle centers, even when $f_{0}(u)$ includes nonlinear decay term.

\section{Appendix E. Supplementary material}

Movies corresponding to Figs. 6, 8, 9, 10, 12, and 13.

Movie captions:

fig6a.mpg Movie corresponding to Fig. 6(a). The major and minor axes of the elliptic camphor particle were $30 \mathrm{~mm}$ and $25 \mathrm{~mm}$, respectively. To confirm the stability of the angular velocity, we added perturbations by poking the camphor particle with tweezers. The time when the elliptic camphor particle was put onto the water surface was set as $t=0 \mathrm{~s}$.

fig6b.mpg Movie corresponding to Fig. 6(b). The major and minor axes of the elliptic camphor particle were $30 \mathrm{~mm}$ and $22.5 \mathrm{~mm}$, respectively. To confirm the stability of the angular velocity, we added perturbations by poking the camphor particle with tweezers. The time when the elliptic camphor particle was put onto the water surface was set as $t=0 \mathrm{~s}$.

fig8a.mpg Movie corresponding to Fig. 8(a). The elliptic camphor particles fixed in arbitrary orientations were released at the same time $(t=97 \mathrm{~s})$. The distance between the centers of 


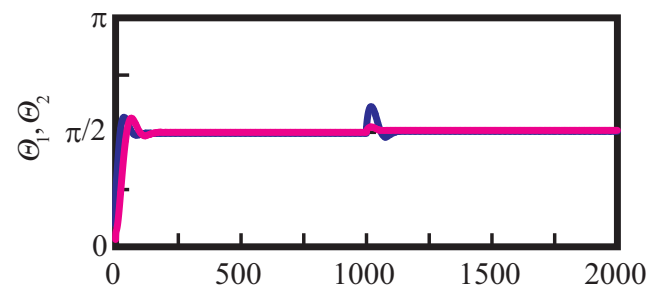

Figure D.16: Results of numerical calculation for the two interacting elliptic camphor particles in the case that $f_{0}(u)$ includes nonlinear decay term in Eq. (D.1). The parameter for friction constant $\eta$ was set as $\eta=0.02$, so that a single particle did not keep moving but stopped. Time series of the angles $\Theta_{1}$ and $\Theta_{2}$ for $\ell=90$ are shown. $\Theta_{1}$ and $\Theta_{2}$ converged to $\pi / 2$, which means that two elliptic camphor particles were aligned by the interaction.

mass was $\ell=30 \mathrm{~mm}$. The time when the elliptic camphor particle was put onto the water surface was set as $t=0 \mathrm{~s}$.

fig8b.mpg Movie corresponding to Fig. 8(b). The case when one of the elliptic camphor particles was poked by tweezers $(t=103 \mathrm{~s})$. The distance between the centers of mass was $\ell=30 \mathrm{~mm}$. The time when the elliptic camphor particle was put onto the water surface was set as $t=0 \mathrm{~s}$.

fig9a.mpg Movie corresponding to Fig. 9(a). The friction constant was set as $\eta=0.08$. The major and minor axes of the elliptic camphor particle were 42 and 35, respectively. To confirm the stability of the angular velocity, we added perturbations at $t=200$.

fig9b.mpg Movie corresponding to Fig. 9(b). The friction constant was set as $\eta=0.12$. The major and minor axes of the elliptic camphor particle were 42 and 35 , respectively. To confirm the stability of the angular velocity, we added perturbations at $t=200$.

fig10a.mpg Movie corresponding to Fig. 10(a). The distance between the centers of mass was $\ell=100$. The friction constant was set as $\eta=0.12$. The major and minor axes of the elliptic camphor particle were 42 and 35, respectively. To confirm the stability of the conformation of the elliptic camphor particles, we added perturbations at $t=1000$.

fig12a.mpg Movie corresponding to Fig. 12(a). The friction constant was set as $\eta=0.15$. The shape of the three-mode shaped camphor particle was $r=40+4 \cos 3 \theta$ in polar coordinates. To confirm the stability of the angular velocity, we added perturbations at $t=200$.

fig12b.mpg Movie corresponding to Fig. 12(b). The friction constant was set as $\eta=0.25$. The shape of the three-mode shaped camphor particle was $r=40+4 \cos 3 \theta$ in polar coordinates. To confirm the stability of the angular velocity, we added perturbations at $t=200$.

fig13a.mpg Movie corresponding to Fig. 13(a). The distance between the centers of mass was $\ell=100$. The friction constant was set as $\eta=0.25$. The shape of the three-mode shaped camphor particle was $r=40+4 \cos 3 \theta$ in polar coordinates. To confirm the stability of the conformation of the elliptic camphor particles, we added perturbations at $t=1000$. 